Pacific Journal of Mathematics

NONISOTROPIC UNITARY SPACES AND MODULES WITH 


\title{
NON-ISOTROPIC UNITARY SPACES AND MODULES WITH CAUCHY-SCHWARZ INEQUALITIES
}

\author{
M. ChaCroN
}

This subject is concerned with non-isotropic unitary spaces $V$ over involutorial division rings $D$ with characteristic not 2 and with non-trivial non-archimedean exponential valuations $w$, which are abelian. It will require a generalized Cauchy-Schwarz inequality relative to $w$. The dimension of $V$ over $D$ need not be finite. Treatments of the unitary module $V_{0}$ of finite vectors $v$ in $v$ (finite, in a technical sense), the ring $L_{0}$ of linear transformations of $V$ that increase lengths, and the unitary group $U$ yield information on the normal subgroup structure of this group and the factor group $U^{(r)} / U^{(r)} \cap Z$, where $U^{(r)}$ is the $r$ th derived group of $U$ and $Z$ is the center of the ground division ring $D$.

Introduction. From a purely ring-theoretic viewpoint this subject arose from the treatment of primitive ring with involution $L$, in which, 2 is invertible and $1-k$ is invertible for every skew-symmetric $k$ in $L$. These invertibility assumptions ensure plenty of unitary elements $u=u^{*-1}$ in $L$, via the Cayley transform $u^{(k)}=(1-k) /(1+k)$ and one is interested in deciding whether or not the factor group $[U, U] /[U, U] \cap Z$ is simple, where $Z=$ center $(L)$ and $U$ is the group of unitary elements in $L$. Another question which is of interest to me is the nature of the ring that is generated by $U$. From a more down to earth viewpoint, this subject specializes to the rings $L$ of the form $L=L(V)$, the full ring of linear transformations of a certain left vector space $V$. It will be assumed throughout that $V$ is a non-isotropic unitary space (in the sense of $\mathrm{I}$. Kaplansky), where the involutorial ground division ring $(D ; *)$ will be equipped with a non-trivial non-archimedean exponential valuation $w$, which is abelian. In fact, $w$ will be a $*$-valuation (in the sense of $\mathrm{S}$. S. Holland, Jr.). I will require, furthermore,

$$
2 w(u \cdot v) \geq w(u \cdot u)+w(v \cdot v)+2 \varepsilon_{0},
$$

where $(\cdot)$ is the form of the unitary space $V, \cdots \leq \cdots$ is the ordering in $G$, the value group of $w$, and $\varepsilon_{0}$ is a constant (depending on $V$ ) in $G$. As shown by a theorem of Kaplansky, if $L$ is as in the outset then $L$ can be represented as a subring of $L(V)$, where the involution in $L$ corresponds to the adjoint involution $\phi \rightarrow \phi^{*}$, provided $L$ has a minimal left ideal. Of 
course, given the valuation of $w$ and/or assumption (1) are extra assumptions.

I shall call $V$ an elliptic space if some inequality (1) holds true. As a special case of (1), there is, of course, the Cauchy-Schwarz inequality obtained by setting $\varepsilon_{0}=0$ in (1):

$$
2 w(u \cdot v) \geq w(u \cdot u)+w(v \cdot v) .
$$

Readers who are more familiar with (2), which has been dealt with recently by Holland [11], may stick to (2) throughout this work. My motivation in (1) will be explained as a concluding remark. I proceed to the material of this work.

This work is organized in four sections of which the first two were kept independent. Treatment of the unitary $R$-module $V_{0}$ of finite vectors cropping up in a given non-degenerate 0 -elliptic space $V$ (e.g. $V$ satisfies (2)) occupies $\$ 1$. Treatment of the ring that is generated by $U$ has been omitted for reasons of space. Instead I will propose the ring $L_{0}$ of linear transformations $\phi$ that increase lengths

$$
w(u \phi \cdot u \phi) \geq w(u \cdot u) \quad(u \in V) .
$$

The ideal structure of this ring will be investigated in an increasingly larger way in $\S \S 2$ and 3. Treatment of the unitary group $U$ of $V$ and related groups will be done also by stages and exclusively in $\S 4$. Now to a synopsis of the results in the order these results will appear in the work.

Concerning Section 1. By length of $v \in V$, I mean the element $w(v \cdot v)$ in the group with infinity adjoined $G \cup\{\infty\}$. Those vectors $v$ having non-negative lengths, or finite vectors, form an $R$-module, where $R$ is the valuation ring in $(D ; w)$. Questions such as when is this module $V_{0}$ a free module, when does $V_{0}$ contain some orthogonal basis and when orthogonal summands of the residue space $\bar{V}$ (in the sense of Holland) can be lifted to $V_{0}$ will be provided satisfactory solutions. In $\$ 1$, Theorem 1.7 (1) will establish that if $V$ has a denumerable basis then $V_{0}$ is free if and only if $V_{0}$ has an orthogonal basis, which relates to Kaplansky's [13, Theorems 37,38 , pp. 46-48]. In the cited theorem it will also be demonstrated that the freeness requirement has a neat number theoretic interpretation, namely, either the value group $G$ is divisible by 2 or $G$ has a first positive element $\varepsilon=0^{+}$such that one at least of these two elements $0, \varepsilon$ is represented by the form (e.g. occurs at length) in each one-dimensional subspace of $V$. As a corollary if 1 (= unity of $D$ ) is represented by the form in each one-dimensional subspace of $V$ then $V_{0}$ has an orthogonal basis. This corollary is due to Holland (see [11, Theorem 5.4]). 
Concerning Section 2. The treatment of the ring $L_{0}$ of all linear transformations $\phi$ that increase lengths corresponds to several needs. For one thing, for these spaces $V$ for which the $R$-module $V_{0}$ is free $L_{0}$ will be shown to be isomorphic to the full endomorphism ring of $V_{0}$ (see $\S 3$, Theorem 3.25). For another, in a continuation of this work, I will show to what extent the ideal structure of $L_{0}$ is the same as that of the subring of $L$ that is generated by $U$. A third need more pressing for this work is to use the ideal structure of $L_{0}$ for the normal subgroup structure of the group $U$. Last but not least one is interested in finding the analog for $L$ of the valuation ring $R$ in $D$. An attempt will be made to keep the treatment of $L_{0}$ coordinate-free. Instead of the usual vector valuation of the ring $L$ viewed as a matrix ring over $D$, I will propose the notion of $*$-prevaluation $\cdots w \geq \cdots$. This is a binary relation between $L$ and $G \cup\{\infty\}$ whose restriction to $Z$ and $G \cup\{\infty\}$ is the same as the binary relation $\underline{w}$ defined by

$$
z \underline{w} \geq g \Leftrightarrow w(z) \geq g .
$$

For general $\phi \in L$ and $g \in G \cup\{\infty\}, \phi w \geq g$ is defined by

$$
w(v \varphi \cdot v \phi) \geq w(v \cdot v)+2 g \quad(v \in V) .
$$

Axioms of a $*$-prevaluation in general are listed and verifications of these axioms for the particular $*$-prevaluation $\cdots w \geq \cdots$ are stated without proofs (or almost). One can view the valuation ring $R$ to be the set of elements $x \in D$ such that $x w \geq 0$. Likewise, $L_{0}$ is the set of linear transformations $\phi$ such that $\phi w \geq 0$. Evidently $U$ is the unitary group of $L_{0}$ so that every 2-sided ideal $I$ of $L_{0}$ which is $*$-closed (e.g. if $\phi \in I$ and if $\phi$ has adjoint $\phi^{*}$ then $\left.\phi^{*} \in I\right)$ gives rise to the congruence subgroup $U \cap(1+I)$. Each $g \geq 0$ in $G$ gives rise to the ideal $L_{g}$ (resp. $L_{g}^{+}$) of all linear transformations $\phi$ such that $\phi w \geq g$ (resp. $\phi w>g$, that is, $w(v \phi \cdot v \phi)>w(v \cdot v)+2 g$, for every $0 \neq v \in V)$. Among the non-formal theorems in $\S 2$, Theorem 2.7, asserts that $L_{0}$ is a prime ring which is an order in an overring of all linear transformations of finite rank, Theorem 2.12, asserts that $L_{0}^{+}$in contained in the Jacobson radical of $L_{0}$ if $V$ is finite-dimensional (or a more general result) and, Theorem 2.19, asserts that the congruence subgroup $U_{0}^{+}=U \cap\left(1+L_{0}^{+}\right)$has all its non-trivial roots of unity (if any) outside $U_{\varepsilon_{0}}^{+}$, for a certain $\varepsilon_{0} \in G$ depending on the characteristics of $D$ and of the residue division ring $\bar{D}$ $\left(\varepsilon_{0}=0\right.$ if $\operatorname{charac}(D) \neq 0 ; \varepsilon_{0}=w(p)$, if $\operatorname{charac}(D)=0$ and $\operatorname{charac}(\bar{D})=$ $p \neq 0 ; \varepsilon_{0}=0$ if $\left.\operatorname{charac}(\bar{D})=0\right)$.

Concerning Section3. This section corresponds to several needs. (i) When the coordinate-free contribution of the $*$-prevaluation $\cdots w \geq \cdots$ as employed in the preceding section is a fact; still, on one or two 
occasions in that section, the matrix delineation of this binary relation is as I believe, inevitable. (ii) In archimedean matrix representation of the $n$-dimensional orthogonal group $O(D ; n(\cdot))$, where $D$ is a field carrying an archimedean exponential valuation and $(\cdot)$ is an $n$-dimensional coordinate orthogonal form on $D$, each orthogonal transformation $\phi$ viewed as a matrix over $D$ has all its entries from the valuation ring $R$ in $D$. In the considered non-archimedean set up, if $\left(f_{i}\right)$ is an orthogonal basis of $V$ and if $\phi \in U$ what can be asserted about the matrix of $\phi$ relative to the basis $\left(f_{i}\right)$ ? (iii) While there is a parallel between $R$ and $L_{0}$ from the formal point of view of $*$-prevaluations can one say something deeper; for instance, is $L_{0}^{+}=\operatorname{Jac}\left(L_{0}\right)$ the unique maximal 2-sided ideal of the ring $L_{0}$ ? (iv) What is the nature of the factor group $U / U_{0}^{+}$? v) Of the mapping $g \rightarrow U_{g}=$ $\left(1+L_{g}\right) \cap U$ ? Taking the situation dealt with in (ii) as a fresh starting point, I will be dealing with those non-isotropic unitary spaces $V$ (inequality (1) not required at the outset, *-valuation $w$ required) equipped with some orthogonal bases $\left(f_{i}\right)$ such that

$$
\phi \in U \Rightarrow w\left(\operatorname{entr}_{i, j}(\phi)\right) \geq \varepsilon_{1} \quad(\text { all } i, j),
$$

where $\varepsilon_{1}$ is a fixed element in $G, \varepsilon_{1} \leq 0$; the case $\varepsilon_{1}=0$ being exactly analogous to the requirement in (ii). In Theorem 3.2, I will show that (6) implies $V$ verifies some inequality (1); in the case $\varepsilon_{1}=0$ and $1 / 2$ exists in $R$, I will establish in Theorem 3.10, that $V$ verifies the usual CauchySchwarz inequality. For general $\varepsilon_{1}$, I will intertwine in Theorem 3.5, the *-prevaluation $\cdots w \geq \cdots$ with its matrix counterpart $\underline{w}$ defined by

$$
\phi \underline{w} \geq g \Leftrightarrow w\left(\operatorname{entr}_{i, j}(\phi)\right) \geq g \quad(\text { all } i, j) .
$$

This approximation theorem uses two parameters, namely an index of ellipticity $\varepsilon_{0}$ and a bound $\varepsilon_{2}$ for the basis $\left(f_{i}\right)$, that is some $\varepsilon_{2} \in G$ such that $\left|w\left(f_{i} \cdot f_{i}\right)-w\left(f_{j} \cdot f_{j}\right)\right| \leq \varepsilon_{2}$ for all pairs $i, j(|g|=g$ if $g \geq 0$ and $|g|=-g$ if $g<0$ ). Both $\varepsilon_{0}$ and $\varepsilon_{2}$ depend fairly sharply on $\varepsilon_{1}$; in case $\varepsilon_{1}=0$ and $1 / 2$ exists in $R$, I will show in Theorem 3.10 that $\varepsilon_{0}=0$ and $\varepsilon_{2}=0$ or else $\varepsilon_{2}$ is the first positive element $\varepsilon$ in $G$. For such a pair $\left(V ;\left(f_{i}\right)\right)$, I will provide satisfactory solutions to the questions in (iii), (iv) and (v) (see Theorems 3.13 through 3.23).

Concerning Section 4. I stated in the opening remarks that my initial motivation for this work can be traced back in the question asking if the group $[U, U] /[U, U] \cap Z$ is a simple group. For instance if $V$ is an Euclidean space (in the sense of E. Artin) of finite dimension $\geq 3$ then by Artin [1, Theorem 5.3, p. 17] the preceding group is a simple group. If, 
contrary to the non-isotropic requirement, $V$ is any non-degenerate unitary space with index $\geq 2$, where the ground division ring $D$ is deprived of the valuation $w$, but $D$ contains at least 25 elements, then by J. Dieudonné [6, Theorems 1 and 4], the cited group is again a simple group. If $V$ is any non-isotropic elliptic space which is orthogonal (e.g. $*=$ identity mapping of $D$ ) and of finite dimension $n \geq 3$ then by Artin [6, Theorem 5.8, p. 184] the group $[U, U] /[U, U] \cap Z$ is not simple. Treatment of the general projective group $U^{(r)} / U^{(r)} \cap Z(r=0,1,2, \ldots)$ for the considered space $V$ arises as a conjunction of the cited results for, at least, $r \leq 1$. In $\S 4$, Theorem 4.4.7, I will establish that $U^{(r)} / U^{(r)} \cap Z$ does not verify the descending chain conditon for normal subgroups at the exception of the obvious cases where $V$ is the 2-dimensional orthogonal space or $V$ is 1-dimensional and $\operatorname{dim}_{Z} D \leq 4$. As an explanation of this negative fact $I$ will propose a positive one, namely, the positive cone $G^{+}$can be dually embedded in the lattice of normal subgroups of the group $U^{(r)} / U^{(r)} \cap Z$ for at least $r \leq 1$. This is done in Theorems 4.2.2 and Theorem 4.3.5, under a certain assumption familiar to $\S 3$, and a different assumption about the residue division ring $\bar{D}$, namely, the dimension of $\bar{D}$ over its own center exceeds 4. As an application of the foregoing theorems, I will derive that every torison normal subgroup of $U$ is central; when $D$ is finite-dimensional or $*$ is of the first kind then the same conclusion will hold for $U$ replaced by $U^{(r)} / U^{(r)} \cap Z$. (See Theorems 4.4 .7 and 4.4.8.)

Concerning assumption (1). In Artin's Geometric Algebra, it is stated that the old principle valid for Euclidean space $V$, namely, "small displacements on the unit sphere can be combined to give arbitrary displacements" (local cit. [1, Chapter V, Sect. 3, p. 179]) fails badly when the ground field $D$ is equipped with a non-archimedean absolute value or, equivalently, a non-archimedean exponential valuation; it suffices to take $V$ to be an orthogonal space of finite dimension $n \geq 3$ with assumption (1). The term used by Artin for such a space $V$ is an elliptic space (see [1, Def. 5.2, p. 180]) - I suggest no explanation of this term. My interest in assumption (1) arises more specifically from Artin's [1, Theorem 5.6, 5.7, pp. 18-183] which asserts that assumption (1) implies and is implied by the requirement some (and, hence, all) congruence subgroup $U_{g}=(1+$ $\left.L_{g}\right) \cap U \neq 1$. Loosely speaking these theorems tell us that (1) has thus a ring-theoretic formulation and, by way of consequence, (1) serves the function of measuring the ring-theoretic incidence of (2). To close, let me add that as of the writing of this article no example of elliptic space $V$ not 
0-elliptic is known to me. $\left({ }^{1}\right)$ As a matter of fact, I will establish in a continuation of this work that if $w$ is of rank 1 then $(1) \Leftrightarrow(2)$, which yields a full ring-theoretic formulation of (2) for such valuations $w$.

1. The module of finite vectors. I will begin with: a review of some of the undefined terms used in the Introduction; the main definitions and conventions; brief comments about non-isotropy, the valuation $w$, and assumption (1) in Introduction. The rest of this section specializes to the modulo $V_{0}$ (Definition 1.1 onward).

(a) Involutorial division ring $D$. Hereafter, $D$ stands for a non-commutative skew-field or division ring with $\operatorname{charac}(D) \neq 2$. Let $x \rightarrow x^{*}$ be any fixed anti-automorphism of $D$ of period 2 if $D$ is not a field and period 1 or 2 otherwise. Let

$$
\begin{gathered}
Z=Z(D) \quad(=\{z \in D \mid z x=x z \vee x \in D\}), \\
\mathscr{U}(D ; *)=\left\{w|x \in D| x x^{*}=1\right\},
\end{gathered}
$$

be the center and unitary group of $D$ respectively. If $x \in D$, denote by $x_{\mathscr{R}}$, the right translation of $D$ induced by $x$ and let

$$
D_{\mathscr{R}}=\left\{x_{\mathscr{R}} \mid x \in D\right\},
$$

be the division ring of right translations of $D$ (regular right representation of $D$ ). Given the symmetric element $s=s^{*}$ in $D, s \neq 0$, let $(s)$ stand for the new involution defined by

$$
x^{(s)}=s x^{*} s^{-1} .
$$

(b) Group with $\infty$ adjoined $G^{\#}$. Hereafter, $G$ stands for an additive group, $G \neq 0$, which is abelian and ordered. Denote the linear ordering on $G$ by $\cdots \leq \cdots$. Extend the addition and the ordering to the set $G^{\#}=G \cup\{\infty\}(\infty \notin G)$ by the laws

$$
\begin{gathered}
g<\infty, \quad \text { for every } g \in G, \quad \text { and } \\
g+\infty=\infty+g=\infty=\infty+\infty, \quad \text { for every } g \in G .
\end{gathered}
$$

(c) *-Valuation ([11, Sect. 2, p. 221]). Following Holland, by *-valuation, I mean a mapping $w: D \rightarrow G^{\#}$ verifying the following.

$$
\text { If } \begin{gathered}
D^{\times}=\{x \in D \mid x \neq 0\}, \text { then } w\left(D^{\times}\right)\left(=\left\{w(x) \mid x \in D^{\times}\right\}\right)=G . \\
w(x y)=w(x)+w(y)(x, y \in D) .
\end{gathered}
$$

\footnotetext{
${ }^{1}$ In a private communication (letter to me of October 3,1984 ) $\mathrm{H}$. Gross, University of Zurich, has elaborated on this matter, where he attributes examples using 2-adic valuation to his student URS-Martin Künzi (Ph.d. dissertation). He also points out that the equivalence $(1) \Leftrightarrow(2)$ has been indeed established by his student in a slightly more general set up.
} 


$$
\begin{gathered}
w(x+y) \geq \operatorname{Min}(w(x), w(y)) . \\
w\left(x^{*}\right)=w(x) .
\end{gathered}
$$

(d) Elliptic space. Let $V$ be any unitary space over the involutorial division ring $D$. Hereafter, $D$ carries a $*$-valuation $w$.

Definition 1.0. I will call $V$ an elliptic space (resp. 0-elliptic space) if for some $\varepsilon_{0}$ in $G$ (resp. $\varepsilon_{0}=0$ )

$$
2 w(u \cdot v) \geq w(u \cdot u)+w(v \cdot v)+2 \varepsilon_{0},
$$

for every pair $u, v$ in $V$.

(e) Concerning the valued division ring $D$. By definition, $w$ is a non-archimedean exponential valuation (in the sense of Jacobson) of $D$, which is abelian since $G$ is abelian and $w$ is non-trivial since $G \neq 0$. Let

$$
\begin{aligned}
& R=\{x \in D \mid w(x) \geq 0\} ; \\
& J=\{x \in D \mid w(x)>0\} .
\end{aligned}
$$

Then $R$ is the valuation ring in $D$. Indeed, $R$ is a subring of $D$, which is preserved under conjugation. Every one-sided ideal of $R$ is 2-sided. Every finite set of ideals of $R$ has a largest member. $J=\operatorname{Jac}(R)$ is the largest non-zero ideal $\neq R$ in $R$. For $g \in G$, let

$$
J_{g}=\{x \in D \mid w(x) \geq g\} .
$$

Then $J_{g}$ is an additive subgroup of $D$; if, further, $g \geq 0$, then $J_{g}$ is an ideal of $R$.

Although 2 is invertible in $D$ it need not be so in $R$. Of course, $2 \in R$ since $R$ has unity. The factor ring $R / J$, a division ring by the preceding, is denoted by $\bar{D}$ (residue division ring):

$$
\bar{D}=R / J \text {. }
$$

(f) Concerning the *-valuation $w$. Inequality (6) carries over to algebraic sums:

$$
w\left(\sum_{i=1}^{n} x_{i}\right) \geq \underset{i=1, \ldots, n}{\operatorname{Min}}\left(w\left(x_{i}\right)\right) \quad \text { (strong triangle inequality). }
$$

In the special case $w\left(x_{l}\right) \neq w\left(x_{j}\right)$ for every pair $i \neq j$, one has the equality

$$
w\left(\sum_{i=1}^{n} x_{i}\right)=\underset{i=1, \ldots, n}{\operatorname{Min}}\left(w\left(x_{i}\right)\right) \quad \text { (special triangle equality), }
$$

Law (7) follows automatically from (5) plus $D$ is finite-dimensional over $Z$ with $*$ of the first kind. This is an immediate corollary to Dieudonné [6, Theorem 5]. 
(g) Non-isotropic form. Hereafter, $V$ stands for any non-degenerate elliptic space. From the elliptic assumption alone follows that if $\operatorname{Rad}(V)$ $=\{v \in V \mid v \cdot V=0\}$, then

$$
\operatorname{Rad} V=\{v \mid v \cdot v=0\} .
$$

From non-degeneracy follows thus non-isotropy of the space $V$ :

$$
v \cdot v=0 \Rightarrow v=0 \quad(v \in V) \text {. }
$$

(h) Constant of ellipticity. If $V \neq 0$ is elliptic then clearly the element $\varepsilon_{0}$ appearing in (8) is not positive. Hence $\varepsilon_{0} \leq 0$. In the sequel, I refer to $\varepsilon_{0}$ as to index of ellipticity without insisting that $\varepsilon_{0}$ be the largest (as a negative element). Throughout this work $V$ stands for a non-degenerate elliptic space and from Theorem 1.6 until the end of this section $V$ is 0-elliptic.

\section{Definition 1.1. Call $v$ a finite vector if for each $u \in V$}

$$
2 w(u \cdot v) \geq(u \cdot u) .
$$

Denote by $V_{0}$, the set of all finite vectors.

Clearly the linear inequality (17) implies

$$
w(v \cdot v) \geq 0
$$

it suffices to set $u=v$ in (17). The quadratic inequality (18) implies back (17) under the 0-elliptic assumption. For the general assumption (1), the penalty is the constant $\varepsilon_{0}$ :

$$
2 w(u \cdot v) \geq w(u \cdot u)+2 \varepsilon_{0} .
$$

Call $v \in V_{0}$ infinitesimal, if

$$
2 w(u \cdot v)>w(u \cdot u) \quad(u \cdot u \neq 0) .
$$

Again, (20) implies

$$
w(v \cdot v)>0,
$$

which implies back (20) up to $\varepsilon_{0}$ :

$$
2 w(u \cdot v)>(u \cdot u)+2 \varepsilon_{0} \quad(u \cdot u \neq 0) .
$$

In the sequel, the subset of all infinitesimals $v$ in $V$ is denoted by $V_{0}^{+}$. In symbols:

(23) $v \in V_{0}^{+} \Leftrightarrow\left(v \in V_{0}\right.$ and $\left.u \cdot u \neq 0 \Rightarrow 2 w(u \cdot v)>w(u \cdot u)\right)$.

THEOREM 1.2. Let $V$ be any unitary space over the $*$-valued division ring $D$-ellipticity of $V$ not required. Then:

(1) If $V_{0}$ is the set of finite vectors on $V$ then $V_{0}$ is a submodule of the $R$-module $V$, where $R$ is the valuation ring in the valued division ring $(D ; w)$. 
(2) If $V_{0}^{+}$is the subset of infinitesimal vectors then $V_{0}^{+}$is a submodule of $V_{0}$.

(3) $V_{0} \cdot V_{0} \subset R$ and, hence, $V_{0}$ can be turned into a unitary module, relative to the form $(\cdot)$.

(4) $V_{0}, V_{0}^{+}$and $J(=\mathrm{Jac}(R))$ are such that

(i) $J V_{0} \subset V_{0}^{+}$,

(ii) $V_{0} \cdot V_{0}^{+} \subset J$, and

(iii) $V_{0}^{+} \cdot V_{0} \subset J$.

Proof. (1) If $v$ is a finite vecor and $\lambda \in R$, then for a given $u \in V$,

$$
\begin{aligned}
2 w(u \cdot(\lambda v)) & =2 w\left((u \cdot v) \lambda^{*}\right)=2 w(u \cdot v)+2 w\left(\lambda^{*}\right) \\
& =2 w(u \cdot v)+2 w(\lambda) \geq 2 w(u \cdot v) \geq w(u \cdot u) ;
\end{aligned}
$$

as this holds for each $u \in V, \lambda v \in V_{0}$ follows. For $u, v$ as before, if $v^{\prime}$ is another finite vector, then

$$
\begin{aligned}
2 w\left(u \cdot\left(v+v^{\prime}\right)\right) & =2 w\left(u \cdot v+u \cdot v^{\prime}\right) \geq 2 \operatorname{Min}\left(w(u \cdot v), w\left(u \cdot v^{\prime}\right)\right) \\
& =\operatorname{Min}\left(2 w(u \cdot v), 2 w\left(u \cdot v^{\prime}\right)\right) \geq w(u \cdot u)
\end{aligned}
$$

implies $v+v^{\prime} \in V_{0}$.

(2) is a trivial adaptation of (1).

(3) If $v, v^{\prime} \in V_{0}$, then from $v \in V_{0}$, and $v^{\prime} \in V_{0} \subset V$, follows $2 w\left(v \cdot v^{\prime}\right) \geq w\left(v^{\prime} \cdot v^{\prime}\right) \geq 0$ so that $w\left(v \cdot v^{\prime}\right) \geq 0$ or, $v \cdot v^{\prime} \in R$, for every pair $v, v^{\prime} \in V$.

(4) (i) If $0 \neq j \in J$, and $0 \neq v \in V_{0}$, then for every $0 \neq u \in V$,

$$
2 w(u \cdot j v)=2 w(u \cdot v)+2 w(j) \geq w(u \cdot u)+2 w(j)>w(u \cdot u)
$$

placing $j v$ in $V_{0}^{+}$.

(ii) If $v \in V_{0}$ and $0 \neq u \in V_{0}^{+}$, then from $u \in V_{0}^{+} \subset V_{0}$ follows $2 w(u \cdot v) \geq w(u \cdot u)$. From $u \in V_{0}^{+}$follows $w(u \cdot u)>0$. Equivalently, $w(u \cdot v)>0$ placing $u \cdot v$ in $J$.

(iii) $V_{0}^{+} \cdot V_{0}=\left(W_{0} \cdot W_{0}^{+}\right)^{*} \subset J^{*}=J$.

As a side remark, if $V \neq 0$ then $V_{0} \neq 0$. This follows trivially from the elliptic axiom. Conversely, in $§ 2$ Theorem 2.18 it is shown that if $V_{0} \neq 0$ then $V$ is elliptic by parts (e.g. every finite dimensional subspace of $V$ satisfies some inequality (1)). I turn to questions about the unitary $R$-module $V_{0}$. As a rule of thumb everything that will follow relies on the following extra assumption, which necessitates the 0-elliptic axiom (2):

$$
V_{0} \backslash V_{0}^{+}=\{v \in V \mid w(v \cdot v)=0\} .
$$


Equivalently, (21) asserts that if $v$ is finite but not infinitesimal then and only then $w(v \cdot v)=0$ (medial vector $v)$.

Definition 1.2. The family $\left(\lambda_{i}\right)_{i \in I}$, where $\lambda_{i} \in D$, is said to be a nullary row vector over $D$ if all $\lambda_{i}=0$ except for finitely many indices in $I$.

In the sequel, by basis of $V$ (resp. $V_{0}$ ) over $D$ (resp. $R$ ) I mean any family $\left(f_{i}\right)_{i \in I}$ in $V$ (in $V_{0}$ ) such that given $v \in V$ (resp. $v \in V_{0}$ ) there is a unique nullary vector $\left(\lambda_{i}\right)$ over $D$ (resp. over $R$ ) such that

$$
v=\sum_{c \in I} \lambda_{i} f_{i}
$$

Can one say that $V_{0}$ is a free module (e.g. $V_{0}$ has some basis)? orthogonally free module (e.g. $V_{0}$ has some orthogonal basis)? The following examples will give some feeling for these questions.

EXAMPLE 1.4. (i) Up to isometry every unitary $R$-module $V_{0}$ where $V$ is 1-dimensional is of the form

$$
V_{0}=\mathscr{I}_{g_{0}}=\left\{x \in R \mid 2 w(x) \geq g_{0}\right\},
$$

where $g_{0} \in w(\mathscr{S}(D ; *))$ and $g_{0} \geq 0$.

(ii) Conversely, for any such $g_{0}$, the set $\mathscr{I}_{g_{0}}$ is a left (in fact, 2-sided) ideal of the valuation ring $R$, which can be realized as the $R$-module $V_{0}$ of some non-degenerate 0 -elliptic space $V$.

(iii) To say that $\mathscr{I}_{g_{0}}$ viewed as a unitary $R$-module (in the sense of (ii)) is orthogonally free it is the same as saying that $\mathscr{I}_{g_{0}}$ is a principal left ideal of the ring $R$ ).

Proof. (1). Let $V$ be any non-degenerate unitary space, which is 1-dimensional. Pick any $v \in V$ such that $v \cdot v \neq 0$. If $s_{1}=v \cdot v$, then $s_{1}=s_{1}^{*} \neq 0$. Replacing $v$ by $\lambda v$ changes $s_{1}$ to $\lambda s_{1} \lambda^{*}$. It follows that one can find some $0 \neq s=s^{*} \notin R$, which is represented by the form (e.g. $s=u \cdot u$, some $u \in V$ ). Then $V$ is isometric to the space $D$ relative to the coordinate form

$$
x \cdot{ }_{s} y=x s y^{*} .
$$

Here, the valuation $w$ is analytic, that is,

$$
w(v \cdot v)=w(\underline{v} \cdot s \underline{v})
$$

where $\underline{v} \in D$ is defined by the equation

$$
v=\underline{v} u \quad(u \cdot u=s) .
$$


Since the space ${ }_{D} D$ is evidently 0 -elliptic relative to $\left({ }_{s}\right)$ it follows that $V$ is 0 -elliptic. Then $V_{0}$ corresponds to $V_{0}\left({ }_{D} D ;\left({ }_{s}\right)\right)$. Now,

$$
x \in V_{0}\left({ }_{D} D ;\left(\cdot{ }_{s}\right)\right) \Leftrightarrow w\left(x s x^{*}\right) \geq 0 \Leftrightarrow 2 w(x) \geq-w(s) .
$$

Thus if $g_{0}=-w(s)$, then $g_{0} \in w(\mathscr{S}(D ; *)), g_{0} \geq 0$, and

$$
V_{0} \cong V_{0}\left({ }_{D} D ;(\cdot s)\right)=\mathscr{I}_{g_{0}} .
$$

(2) Pick any $s=s^{*}$ such that $w(s)=-g_{0}$. Equip ${ }_{D} D$ with the form

$$
x \cdot y=x s y^{*} .
$$

By construction, $V={ }_{D} D$ is a non-degenerate 0 -elliptic space with $V_{0}=\mathscr{I}_{g_{0}}$.

(3) Since $\mathscr{I}_{g_{0}}$ is an $R$-submodule of a 1-dimensional non-degenerate unitary space it is clear that $\mathscr{I}_{g_{0}}$ cannot contain any two non-zero orthogonal vectors. Hence, $\mathscr{J}_{g_{0}}$ is 1-dimensional. Equivalently, the left ideal $\mathscr{I}_{g_{0}}$ is a principal left ideal.

EXAMPLE 1.5. (a) If $\left(f_{\imath}\right)$ is any orthogonal basis of the space $V$, then for every $v \in V$

$$
v=\sum_{\iota \in I}\left(v \cdot f_{\iota}\right) \frac{1}{f_{\imath} \cdot f_{\iota}} f_{\iota} .
$$

(b) If $V$ possesses some orthogonal basis and if every $g \in G$ that is represented by the form (e.g. there is $v \in V$ such that $v=w(v \cdot v)$ ) is divisible by 2 then the $R$-module $V_{0}$ is orthogonally free.

Proof. (a) For let $\left(\lambda_{\iota}\right)$ be the nullary vector over $D$ such that $v=\sum \lambda_{\phi i}$. By construction, there is a unique finite subset $I_{1}$ of $I$ such that $\lambda_{\iota} \neq 0$ for each $\iota \in I_{1}$ and $\cdot v=\sum_{\iota \in I_{1}} \lambda_{\iota} f_{\iota}$. If

$$
v^{\prime}=\sum_{\iota \in I_{1}}\left(v \cdot f_{\iota}\right) \frac{1}{f_{\iota} \cdot f_{\iota}} f_{\iota}
$$

then $v-v^{\prime}$ is orthogonal to each $f_{l}$. Hence $v=v^{\prime}$.

(b) Let $\left(f_{\iota}\right)$ be any orthogonal basis of $V$ over $D$. Without disturbing the orthogonality relations each $f$ can be replaced by $g_{\imath}$ with $w\left(g_{\imath} \cdot g_{\imath}\right)=0$. The new basis $(g)$ is in $V_{0}$. If $v \in V_{0}$ then by (a),

$$
v=\sum_{\imath \in I}\left(v \cdot g_{\imath}\right) \frac{1}{g_{\imath} \cdot g_{\imath}} g_{\imath}
$$

follows. If $v \cdot g_{\imath} \neq 0$ then from 0 -ellipticity follows

$$
2 w\left(v \cdot g_{\iota}\right) \geq w(v \cdot v)+w\left(g_{\imath} \cdot g_{\iota}\right)=w(v \cdot v) \geq 0
$$

giving $w\left(v \cdot g_{\iota}\right) \geq 0$ or, $v \cdot g_{i} \in R$. Hence, $\left(g_{\iota}\right)$ is a basis of $V_{0}$ over $R$. 
The treatment of bases of the $R$-module $V_{0}$ (if any) necessitates, of course, treatment of those $v \in V_{0}$ such that $\lambda v \in V_{0}$ implies $\lambda \in R$. Before I deal with such vectors $v$ let me observe that if in the value group $G$ there is some $g_{0}>0$ such that $g_{0}<2 g$ for every $g>0$ then $G$ has a first positive element $\varepsilon$ which is precisely $g_{0}$. Indeed, if $g<0$ but $g<g_{0}$, then $g_{0}-g>0$ so that $g_{0}<2\left(g_{0}-g\right)$, that is, $2 g<g_{0}$, a contradiction.

THEOREM 1.6. Let $V$ be any nondegenerate 0 -elliptic space, let $v \in V$ and let $g_{0}=w(v \cdot v)$. The following requirements are equivalent.

(1) $g_{0}<2 g$ for every $g \in G^{+}(=\{h \in G \mid h>0\})$.

(2) $g_{0}=0$ or $g_{0}=\varepsilon=1$ st positive element of $G$.

(3) $g_{0} \geq 0$ and if $\lambda v \in V_{0}$, then $\lambda \in R$,

(4) $g_{0} \geq 0$ and if $u \in V_{0}$, then $w(u \cdot u) \geq g_{0}$.

If one (and, hence all) requirements are verified then $I$ will call $v a$ pseudo-medial vector.

Proof. (1) $\Rightarrow(2)$. If $v$ is not medial then $0<g_{0}<2 g$ for every $g \in G^{+}$. From this $g_{0}=\varepsilon$.

(2) $\Rightarrow$ (3) From $\lambda v \in V_{0}$ follows that if $g=w(\lambda)$ then $2 g+g_{0} \geq 0$ or $\varepsilon=g_{0} \geq-2 g$; equivalently, $-g \leq 0$, that is, $g \geq 0$ or $\lambda \in R$.

(3) $\Rightarrow$ (4) Given $u \in V_{0}$ if $u_{1}$ is the projection of $u$ on $v$ :

$$
u_{1}=(u \cdot v) \frac{1}{v \cdot v} v
$$

then $w\left(u_{1} \cdot u_{1}\right)=2 w(u \cdot v)-w(v \cdot v) \geq w(u \cdot u) \geq 0$ placing $u_{1}$ in $v_{0}$. If $\lambda=(u \cdot v)(v \cdot v)$ then since $\lambda v=u_{1} \in V_{0}, \lambda \in R$ follows or $w(u \cdot v) \geq$ $w(v \cdot v)$.

(4) $\Rightarrow$ (1) Let $g \in G$. If $g_{0} \geq 2 g$ then if $x \in D$ is such that $w(x)=$ $g$ then $w\left(x^{-1} v \cdot x^{-1} v\right)=g_{0}-2 g \geq 0$ placing $x^{-1} v$ in $V_{0}^{+} \subset V_{0}$. Thus $w\left(x^{-1} v \cdot v\right)=g_{0}-g \geq w(v \cdot v)=g_{0}$ or $g \leq 0$. The contrapositive of this is that if $g>0$ then $g_{0}<2 g$, as desired.

THEOREM 1.7. Let $V$ be any non-degenerate 0-elliptic space, which has some denumerable basis. The following requirements are equivalent.

(1) The $R$-module $V_{0}$ is a free module (e.g. $V_{0}$ has some basis over $R$ )

(2) The $R$-module $V_{0}$ is orthogonally free (e.g. $V_{0}$ has some orthogonal basis over $R$ ). 
(3) For every $g \in G$ which is represented by the form (e.g. $g=w(v \cdot v)$ for some $v \in V$ ) there is $g^{\prime} \in G$ and $\varepsilon \in G$ such that $g=2 g^{\prime}+\varepsilon$, where either $\varepsilon=0$ or else $\varepsilon$ is the first positive element in $G$.

(4) Given $0 \neq v \in V$ there is $\lambda \in D$ such that $\lambda v$ is a pseudo-medial vector ( $V$ contains enough pseudo-medial vectors).

Proof. (1) $\Rightarrow$ (2) Evidently the $R$-module $V$ is an essential extension of the $R$-module $V_{0}$ in that if $v \in V$ there is $0 \neq \lambda \in D$ such that $\lambda v \in V_{0}$. Equivalently, $V=D V_{0}$. Then every basis of $V_{0}$ is a basis of $V$ over $D$. Hence $V_{0}$ contains a denumerable basis, say, $\left(e_{i}\right)_{i=1,2, \ldots}$. Now $V$ is non-isotropic. Thus by induction on $n$ one can define the following sequence $\left(f_{i}\right)_{i=1,2, \ldots}$ :

$$
f_{1}=e_{1}, \quad f_{n}=e_{n}-\sum_{i=1}^{n} e_{n} \cdot f_{i}\left(\frac{1}{f_{i} \cdot f_{i}}\right) f_{i} \quad(n>1) .
$$

Put:

$$
\alpha_{n, i}=\left(e_{n} \cdot f_{i}\right) \frac{1}{f_{i} \cdot f_{i}} \quad(i<n),
$$

$v_{n, i}=\alpha_{n, i} f_{i}$. It is to be shown that $v_{n, i} \in V_{0}$. For since $V$ is 0 -elliptic it follows that $2 w\left(e_{n} \cdot f_{i}\right)-w\left(f_{i} \cdot f_{i}\right) \geq w\left(e_{n} \cdot e_{n}\right) \geq 0\left(e_{n} \in V_{0}\right)$. Now, by a straight calculation $w\left(v_{n, i} \cdot v_{n, i}\right)=2 w\left(\alpha_{n, i}\right)+w\left(f_{i} \cdot f_{i}\right)=2 w\left(e_{n} \cdot f_{l}\right)-$ $w\left(f_{i} \cdot f_{i}\right)$. The relations $v_{n, i} \in V_{0}$ will be used to show that, in turn, $\alpha_{n, i} \in R$ and $f_{n} \in V_{0}$ for every pair $i, n$ with $i<n$. From the preceding $\alpha_{n, i} f_{i}=v_{n, i} \in V_{0}$. By a straight induction on $n$ follows $f_{n}=e_{n}$ $\sum_{i=1}^{n-1} \alpha_{n i} f_{i} \in V_{0}$. For the relations $\alpha_{n i} \in R$, where $i<n$, use the $n-1$ first equations of the $f_{j}$

$$
\begin{aligned}
f_{1}= & e_{1}, \\
f_{2}= & e_{2}-\alpha_{2,1} f_{1}, \\
& \ldots \\
f_{n-1}= & e_{n-1}-\sum_{i=1}^{n-2} \alpha_{n-1, i} f_{i},
\end{aligned}
$$

to express the $f_{i}, 1 \leq i \leq n-1$, in terms of the $e_{j}, 1 \leq j \leq n-1$. Since the $f_{i}$ with $1 \leq i \leq n-2$ evidently do not use $e_{n-1}$ it follows that

$$
f_{n-1}=\beta_{1} e_{1}+\cdots+\beta_{n-2} e_{n-2}+1 \cdot e_{n-1},
$$

for some $\beta_{i} \leq D$. In the equation of $f_{n}$,

$$
f_{n}=e_{n}-\alpha_{n, 1} f_{1}-\cdots-\alpha_{n, n-1} f_{n-1},
$$


substituting for the $f_{i}$ with $1 \leq i \leq f_{n-1}$ one obtains

$$
f_{n}=\gamma_{1} e_{1}+\cdots+\gamma_{n-2} e_{n-2}-\alpha_{n, n-1} e_{n-1}+e_{n},
$$

where the $\gamma_{i} \in D, 1 \leq i \leq n-2$. Since $e, \ldots, e_{n}, \ldots$ is a basis of the $R$-module $V_{0}$ it follows that all coefficients in the preceding equation are in $R$, particularly $-\alpha_{n, n-1} \in R$. Hence,

$$
f_{n}+\alpha_{n, n-1} f_{n-1}=f_{n}^{\prime}=e_{n}-\alpha_{n 1} f_{1}-\cdots-\alpha_{n, n-2} f_{n-2}
$$

where $f_{n}^{\prime} \in V_{0}^{\prime}$. Repeating the preceding argument for $f_{n}$ replaced by $f_{n}^{\prime}$ shows that $\alpha_{n, n-2} \in R$. Step by step $\alpha_{n, n-3}, \ldots, \alpha_{n, 1} \in R$ follow.

Since the $\alpha_{n i}$ are all in $R$ it follows that the $e_{j}$ are $R$-expressible in terms of the $f_{i} \in V_{0}$. Since the $e_{j}$ span $V_{0}$ so will be the $f_{i}$ and since the $f_{i}$ are orthogonal they form hence an orthogonal basis of $V_{0}$ over $R$.

$(2) \Rightarrow(3)$. I will first establish the following identity, which was suggested to me by the referee:

$$
\begin{aligned}
w\left(\sum_{i=1}^{r} v_{i} \cdot v_{i}\right)= & \operatorname{Min}_{j=1, \ldots, r}\left(w\left(v_{j} \cdot v_{j}\right)\right) \\
& \left(0 \neq v_{1}, \ldots, v_{r} \text { orthogonal vectors in } V\right) .
\end{aligned}
$$

One-half of (22) follows automatically from the generalized triangle inequality. Conversely, for any fixed $i$,

$$
2 w\left(v_{i} \cdot v_{i}\right)=2 w\left(v_{i} \cdot \sum_{j=1}^{r} v_{j}\right) \geq w\left(v_{i} \cdot v_{i}\right)+w\left(\sum_{j=1}^{r} v_{j} \cdot v_{j}\right)
$$

giving after cancellation by $w\left(v_{i} \cdot v_{i}\right), w\left(v_{i} \cdot v_{i}\right) \geq w\left(\sum_{j=1}^{r} v_{j} \cdot v_{j}\right)$. As this holds for every $i$ it follows that $\operatorname{Min}_{i=1, \ldots, r}\left(w\left(v_{i} \cdot v_{i}\right)\right) \geq w\left(\sum_{j=1}^{r} v_{j} \cdot v_{j}\right)$. Next, I observe that if $\left(f_{i}\right)$ is any orthogonal basis of the $R$-module $V_{0}$ then by a straight adaptation of the preceding if $\left(\lambda_{l}\right)$ is any nullary row vector over $D$, then

$$
w\left(\sum_{i \in I} \lambda_{i} f_{i} \cdot \lambda_{i} f_{i}\right)=\operatorname{Min}_{i \in I} w\left(\lambda_{\iota} f_{\iota} \cdot \lambda_{\iota} f_{\iota}\right)
$$

$\left(\left(f_{i}\right)\right.$ any orthogonal family in $\left.V\right)$. Now since $\left(f_{i}\right)$ is a basis of $V_{0}$ over $R$ it follows that for each fixed $i \in I, f_{i} \in V_{0}$ and if $\lambda f_{i} \in V_{0}$ then $\lambda \in R$. In view of Theorem 1.6, $f_{i}$ is then a pseudo-medial vector of $V$ for every $i \in I$. If then $\infty \neq g$ is represented by the form, say, $g=w(v \cdot v)$ then for some $0 \neq \lambda^{\prime} \in D, \lambda^{\prime} v \in V_{0}$. If $v^{\prime}=\lambda^{\prime} v$ then $v^{\prime}=\sum \lambda_{i} f_{i}$ for a certain nullary row vector $\left(\lambda_{i}\right)$ over $R$. From (30), follows

$$
w\left(v^{\prime} \cdot v^{\prime}\right)=w\left(\lambda_{0}\left(f_{i_{0}} \cdot f_{i_{0}}\right) \lambda_{0}^{*}\right)
$$


for some $i_{0} \in I$ and some $\lambda_{0} \in R$. Since $v \neq 0$ and $\lambda^{\prime} \neq 0$ it follows that $v^{\prime} \neq 0$ so that $w\left(v^{\prime} \cdot v^{\prime}\right) \neq \infty$ and, hence, $\lambda_{0} \neq 0$. Then

$$
\begin{aligned}
g & =w\left(v^{\prime} \cdot v^{\prime}\right)-2 w\left(\lambda^{\prime}\right)=w\left(\lambda_{0}\left(f_{i_{0}} \cdot f_{i_{0}}\right) \lambda_{0}^{*}\right)-2 w\left(\lambda^{\prime}\right) \\
& =2 w\left(\lambda_{0} \lambda^{\prime-1}\right)+\varepsilon
\end{aligned}
$$

where $\varepsilon=f_{i_{0}} \cdot f_{i_{0}}=0$ or else $\varepsilon$ is the first positive element in $G$ (Theorem 1.6).

(3) $\Rightarrow(4)$ This is a corollary to Theorem 1.6.

(4) $\Rightarrow(1)$ That $V$ possesses an orthogonal basis as soon as $V$ possesses a denumerable basis was shown for the $R$-submodule $V_{0}$ itself so, $V$ has some orthogonal basis. Given any orthogonal basis $\left(g_{i}\right)_{i \in I}$ for the space $V$ over $D$, scale this basis to an orthogonal basis of $V$ over $D$, where the new $g_{i}$ are pseudo-medial vectors. I assert that these $g_{i}$ form a basis of $V_{0}$ over $R$. For to begin with the $g_{i} \in V_{0}$. They are linearly independent over $D$ and, hence, over $R$. To see that the $g_{i} \operatorname{span} V_{0}$ over $R$ proceed as follows. Given $0 \neq v \in V_{0} \subset V$, since $\left(g_{i}\right)_{i \in I}$ is an orthogonal basis in $V$, it follows (Example 1.5) that

$$
v=\sum_{i \in I}\left(v \cdot g_{i}\right)\left(\frac{1}{g_{i} \cdot g \cdot i}\right) g_{i},
$$

where it is understood that all the $\left(v \cdot f_{l}\right)\left(f_{\imath} \cdot f_{\imath}\right)=0$ but for a finite number of indices. Since each $g_{\imath}$ is pseudo-medial $w\left(v \cdot g_{\imath}\right) \geq w\left(g_{i} \cdot g_{l}\right)$ follows (Theorem 1.7, point 4.) or, $\left(v \cdot g_{i}\right)\left(g_{\imath} \cdot g_{\imath}\right) \in R$. Hence the preceding equation shows that $v$ is $R$-expressible in terms of the $g_{i}$, as desired.

As stated earlier in Introduction, the equivalence $1 \Leftrightarrow 2$ holds for any unitary $R$-module $V_{0}$, where $R$ is any valuation ring containing $1 / 2$ and $V_{0}$ is finite-dimensional (cf. [13; Theorems 37, 38]). Note, however, that the argument as given in [13] makes essential use of these two extra assumptions (extra assumptions for Theorem 1.7).

Using arguments similar to the argument in Theorem 1.7 and standard arguments one can show the following corollaries.

COROLlaRY 1.8. Let $V$ be any non-degenerate 0-elliptic space. Then:

(1) If $V$ has some orthogonal basis then $V_{0}$ is orthogonally free if and only if $V$ contains enough pseudo-medial vectors.

(2) If the subgroup $2 G$ is of index $\leq 2$ in $G$ (in particular if $G$ is isomorphic to the ordered additive group of integers) then given $g \in G$ there 
is $g^{\prime} \in G$ and $\varepsilon$ such that $g=2 g^{\prime}+\varepsilon$, where either $\varepsilon=0$ or $\varepsilon$ is the first positive element in $G$ so that $V$ contains enough pseudo-medial vectors.

(3) If $V$ is spanned by pseudo-medial vectors it does not generally follow that $V$ contains enough pseudo-medial vectors.

COROllary 1.9. Let $V$ be as in Corollary 1.8. Then:

(1) If $V_{0}^{+}$is an orthogonal summand in $V_{0}$ then, in fact, $V_{0}=V_{0}^{+}$.

(2) If: $V_{0}^{+}$has denumerable basis as an $R$-module then so must be $V_{0}$.

To find orthogonal summands of the $R$-module $V_{0}$ less forbidding than $V_{0}^{+}$, I will first recall a construction due to Holland of the residue space $\bar{V}$ ([11, Theorem 5.4]). Let $\bar{V}=V_{0} / V_{0}^{+}$. Then annihilator in $R$ of $\bar{V}$ can be turned into a left space over $\bar{D}=R / J$. By Theorem 1.2, again,

$$
V_{0}^{+} \cdot V_{0} \subset J
$$

so that $\bar{V}$ can be equipped with the form

$$
\left(v+V_{0}^{+}\right) \cdot\left(u+V_{0}^{+}\right)=v u+J\left(v, u \in V_{0}\right) .
$$

Then $(\bar{V} ;(\cdot))$ is a unitary space over $\bar{D}$, relative to the induced involution of $\bar{D}$. By construction, if $\bar{v} \cdot \bar{v}=0$ then for $\bar{v}=v+V_{0}^{+}, v \cdot v \in J$ follows so that $v \in V_{0}^{+}$or $\bar{v}=0$. Thus $\bar{v}$ is non-isotropic. This is the

REMARK 1.10 (Holland [11, Theorem 5.4]). Let $V$ be any unitary space over the $*$-valued division ring $D$. Then the factor $R$-module $V_{0} / V_{0}^{+}$can be turned into a non-isotropic unitary space over the residue division ring $\bar{D}$ (residue space $\left.\bar{V}=\left(V_{0} / V_{0}^{+} ;(\cdot)\right)\right)$, relative to the form

$$
\left(v+V_{0}^{+}\right) \cdot\left(u+V_{0}^{+}\right)=v \cdot u+J \text {. }
$$

THEOREM 1.11. Let $V$ be any non-degenerate 0-elliptic space having some orthogonal basis. Let $\bar{V}^{(1)}$ be any non-zero finite dimensional subspace of the residue space $V$. Then $\bar{V}^{(1)}$ can be lifted to an $R$-submodule $V_{0}^{(1)}$ of the $R$-module $V_{0}$ such that:

(1) $V_{0}^{(1)} / V_{0}^{+}=\bar{V}^{(1)}$.

(2) $V_{0}^{(1)}$ possesses an orthogonal basis formed by medial vectors with cardinality equal to $\operatorname{dim}_{\bar{D}} \bar{V}^{(1)}$.

(3) $V_{0}^{(1)}$ is an orthogonal summand of $V_{0}$ with orthogonal complement $V_{0}^{(2)}$ mapping onto the orthogonal of $\bar{V}^{(1)}$ in $\bar{V}$.

Proof 1. and 2. Let $\bar{f}_{1}, \ldots, \bar{f}_{n}$ be a basis of $\bar{V}^{(1)}$ over $\bar{D}$ and let $f_{1}, \ldots, f_{n} \in V_{0}$ map onto $\bar{f}_{1}, \ldots, \bar{f}_{n}$ respectively. Denote by $V_{0}^{(1)}$ the $R$-submodule of $V_{0}$ that is spanned by the $f_{i}$. By construction, $V_{0}^{(1)} / V_{0}^{+}=\bar{V}^{(1)}$. 
Also, the $f_{i}$ form a basis of the $R$-module $V_{0}^{(1)}$. For let $\lambda_{1}, \ldots, \lambda_{n} \in R$ be such that $\sum \lambda_{i} f_{i}=0$. Then $\sum \bar{\lambda}_{i} \bar{f}_{i}=0$. Thus $\lambda_{i} \in J$ for every $i$. If some $\lambda_{i} \neq 0$ pick one of least value, say, $\lambda_{1}$. Then $f_{1}+\sum_{i>1} \lambda_{1}^{-1} \lambda_{i} f_{i}=0$. By construction, $\lambda_{1}^{-1} \lambda_{i} \in R$. The preceding argument shows that $\bar{f}_{1}+$ $\sum_{\iota>1} \overline{\lambda_{1}^{-1} \lambda_{i}} \bar{f}_{i}=0$, which is nonsense. This shows that all $\lambda_{i}=0$. Therefore $\left(f_{i}\right)$ is a basis $V_{0}^{(1)}$ over $R$. I proceed to show that the basis $\left(f_{i}\right)$ can be transformed to an orthogonal basis $\left(g_{i}\right)$ of $V_{0}^{(1)}$, where each $g_{i}$ is a medial vector. Put:

$$
\begin{aligned}
& g_{1}=f_{1}, \\
& g_{2}=f_{2}-\left(f_{2} \cdot g_{1}\right) \frac{1}{g_{1} \cdot g_{1}} g_{1}, \\
& \quad \ldots \\
& g_{n}=f_{n}-\sum_{i<n}\left(f_{n} \cdot g_{i}\right) \frac{1}{g_{l} \cdot g_{i}} g_{i} .
\end{aligned}
$$

I claim that $\left(g_{i}\right)$ is the desired basis. The agrument goes by induction on $n$. For $n=1, g_{1}=f_{1} \in V_{0}^{(1)} \subset V_{0}$ and $g_{1} \notin V_{0}^{+}$. Thus $g_{1}$ is pseudo-medial. Assume that $g_{1}, \ldots, g_{n-1}$ are medial vectors. Since for each $i<n$, $g_{i}$ is medial and since $f_{n} \in V_{0}^{(1)} \subset V_{0}$, it follows by Theorem 1.7, that $\left(f_{n} \cdot g_{i}\right)\left(g_{\imath} \cdot g_{i}\right) \in R$. Hence, $\sum_{i<n}\left(f_{n} \cdot g_{i}\right)\left(1 /\left(g_{i} \cdot g_{i}\right) g_{i} \in V_{0}^{(1)}\right)$. Thus $g_{n} \in V_{0}^{(1)} \subset V_{0}$. Assume that $g_{n} \in V_{0+\cdot}$. In the equation

$$
g_{n}=f_{n}-\sum_{i<n}\left(f_{n} \cdot g_{i}\right) \frac{1}{g_{i} \cdot g_{i}} g_{\iota}
$$

substituting for all the $g_{i}$ with $i<n$ in terms of the $f_{j}$ will not disturb the term $f_{n}$ since each $g_{i}$ is $R$-expressible in terms of $f_{j}$ with $j \leq i \leq n$. Going down to the residue space $\bar{V}$, one has

$$
0=\bar{f}_{n}+\sum_{i<n} \bar{\gamma}_{i} \bar{f}_{i} \quad\left(\gamma_{\iota} \in \bar{D}\right)
$$

contrary to the fact that the $\left(\bar{f}_{j}\right)_{j \in I}$ form a basis over $\bar{D}$. This shows that $g_{n} \notin V_{0+}$ and, hence, $g_{n} \in V_{0}^{(1)} \subset V_{0}$ is a medial vector. By construction, the sequence $g_{1}, \ldots, g_{n}$ is an orthogonal sequence of medial vectors in $V_{0}^{(1)}$. Since the $f_{i}$ are $R$-expressible in terms of the $g_{j}$ and since the $f_{\iota}$ span $V_{0}^{(1)}$ it follows that $g_{1}, \ldots, g_{n}$ is a basis of $V_{0}^{(1)}$ over $R$.

(3) It is to be shown that if $V^{(2)}=V_{0}^{(1)^{\perp}}$ is the orthogonal of $V_{0}^{(1)}$ in $V$ then $V_{0}^{(2)}=V_{0}^{(1)^{\perp}} \cap V_{0}$ is an orthogonal complement of $V_{0}^{(1)}$ in $V_{0}$.

Since $V_{0}^{(1)^{\perp}}=\left\{g_{1}, \ldots, g_{n}\right\}^{\perp}$ it follows that $V^{(2)} \oplus D V_{0}^{(1)}=V$. Since $V_{0}^{(1)}$ has an orthogonal basis $g_{1}, \ldots, g_{n}$ as an $R$-module it follows that the projection of any given $v \in V_{0} \subset V$ on $D V_{0}^{(1)}$ is

$$
v_{1}=\sum_{i=1}^{n}\left(v \cdot g_{\iota}\right)\left(1 /\left(g_{\imath} \cdot g_{\iota}\right) g_{i}\right) \text {. }
$$


Thus

$$
v=v_{1} \oplus v_{2}, \quad\left(v_{1} \in D V_{0}^{(1)} ; v_{2} \in V^{(2)}\right) .
$$

Now the $g$ are medial vectors and $v \in V_{0}$. By Theorem 1.7, again, follows $\left(v \cdot g_{\imath}\right)\left(g_{\imath} \cdot g_{\imath}\right) \in R$. Hence

$$
v_{1}=\sum_{i}\left(v \cdot g_{\imath}\right) \frac{1}{g_{\imath} \cdot g_{\imath}} g_{\imath} \in V_{0}^{(1)} .
$$

Thus $v_{2}=v-v_{1} \in V_{0}$ so that $v_{2} \in V_{0}^{(2)}$. Therefore $V_{0}=V_{0}^{(1)} \oplus V_{0}^{(2)}$.

(4) $V_{0}^{(2)}$ maps onto the orthogonal $\bar{V}^{(2)}$ of $V^{(1)}$ in $\bar{V}$.

Clearly, $\bar{V}^{(1)} \oplus \bar{V}^{(2)}=\bar{V}$. Since $V_{0}^{(1)} \oplus V_{0}^{(2)}=V_{0}$ it follows that $\bar{V}_{0}^{(1)}$ $\oplus \bar{V}_{0}^{(2)}=\bar{V}_{0}=\bar{V}$. By construction, $\bar{V}_{0}^{(1)}=\bar{V}^{(1)}$. Hence, $\overline{V^{(1)}} \oplus \overline{V_{0}^{(2)}}=\bar{V}$. Then $\bar{V}_{0}^{(2)} \subset \bar{V}^{(1)^{\perp}}=\bar{V}^{(2)}$ forces $\bar{V}_{0}^{(2)}=V^{(2)}$.

THEOREM 1.12. Let $V$ be any non-degenerate 0-elliptic space, which is finite-dimensional. Then:

(1) Any two maximal orthogonal families of medial vectors have equal cardinalities $n_{1}$.

(2) Any two maximal orthogonal families of truly pseudo-medial vectors have equal cardinalities $n_{2}$ provided $V$ contains enough pseudo-medial vectors.

(3) $n_{1}+n_{2}=\operatorname{dim}_{D} V$.

Proof 1. Let $f_{1}, \ldots, f_{n_{1}}$ be any maximal orthogonal family of medial vectors. Denote by $\bar{V}^{(1)}$ the span of the $\bar{f}_{i}$ in $\bar{V}$. Clearly $\bar{f}_{1}, \ldots, \bar{f}_{n_{1}}$ is a basis of $\overline{V^{(1)}}$ over $\bar{D}$. If $V_{0}^{(1)}$ is the span of the $f_{i}$ over $R$ then by Theorem 1.11, $V_{0}^{(1)}$ is an orthogonal summand of $V_{0}$. Assume that $n_{1} \neq \operatorname{dim}_{\bar{D}} \bar{V}$. by the cited theorem, the orthogonal of $V_{0}^{(1)}$ in $V_{0}$ maps onto $\bar{V}^{(1)^{\perp}}$. Since $n_{1} \leq \operatorname{dim}_{\bar{D}} \bar{V}$ and since $\bar{V}$ is non-isotropic and, hence, non-degenerate, it follows that $\overline{V^{(1) \perp}} \neq 0$ so that $\overline{V^{(1)^{\perp}}}$ contains some non-zero vector $\bar{f}$. If $f \in\left(V_{0}^{(1)}\right)^{\perp}$ maps onto $\bar{f}$ then $f$ is a medial vector. Now $f_{1}, \ldots, f_{n_{1}}$, is an orthogonal family consisting solely of medial vectors, a contradiction. This shows that $n_{1}=\operatorname{dim}_{\bar{D}}(\bar{V})$.

(2) Let $g_{1}, \ldots, g_{n_{2}}$ be any maximal orthogonal family of truly pseudomedial vectors. Let $V_{0}^{(2)}$ be the span of the $g_{i}$ over the ring $R$. I claim that $V_{0}^{(2)}$ is an orthogonal summand of the $R$-module $V_{0}$. For let $v \in V_{0}$. As a vector in $V, v=v_{1} \oplus v_{2}$, where $v_{2}$ is the projection of $v$ onto the subspace $D V_{0}^{(2)}$, which has the orthogonal basis $g_{n_{1}}, \ldots, g_{n_{2}}$. Now,

$$
v_{2}=\sum_{i=1}^{n_{2}}\left(v_{2} \cdot g_{i}\right)\left(\frac{1}{g_{\imath} \cdot g_{i}}\right) g_{i} \text {. }
$$


Since $g_{i}$ is pseudo-medial it follows by Theorem 1.7 that $\left(v_{2} \cdot g_{i}\right)\left(g_{\imath} \cdot g_{i}\right)$ $\in R$. Hence, $v_{2} \in V_{0}^{(2)}$. From this $v_{1}=v-v_{2} \in V_{0}$. Hence $V_{0}^{(2)}$ is an orthogonal summand in $V_{0}$. Let $V_{0}^{(1)}$ be its orthogonal complement. Every $0 \neq v \in V_{0}^{(1)}$ can be scaled to a pseudo-medial vector $\lambda v \in V_{0}$. By construction, $\lambda v \in V_{0}^{(1)}$. By maximality of the family $g_{1}, \ldots, g_{n_{2}}$ follows $\lambda v$ is a medial vector. Therefore, $V_{0}^{(1)}$ possesses an orthogonal basis of medial vectors $f_{1}, \ldots, f_{n_{1}^{*}}$. Going down to the residue space $\bar{V}$ one finds that $n_{1}^{*}=\operatorname{dim}_{\bar{D}} \bar{V}=n_{1}$. Therefore $n_{2}=n-n_{1}$.

2. Ring of linear transformations increasing lengths. The goal of this section is two-fold. Firstly, a parallel between the valuation ring $R$ in $D$ and a certain subring $L_{0}$ of the full ring $L$ of linear transformations of the space $V$ is drawn. Here $V$ is any non-degenerate elliptic space and the parallel is obtained by means of a certain binary relation $\cdots w \geq \cdots$ (hereafter called *-prevaluation) between $L$ and the value group $G$. Secondly, the ideal structure of the ring $L_{0}$ is dealt with with a view to apply the results to the normal subgroup structure of the group $U$. At the end of the section certain torsion-free congruence subgroup of $U$ is pointed out. I will begin with recalling one or two facts about $L$.

(a) Notation. Members of $L$ generally written $\phi, \sigma, \tau, \ldots$ always operate on the right of their domain, which is the space $V$.

(b) Ideal of linear transformations of finite rank. If $\phi \in L$ is such that $V \phi$ is finite-dimensional then $\phi$ is a linear transformation of finite rank. Let

$$
\mathscr{F} L=\{\phi \in L \mid \operatorname{dim} V \phi<\infty\} .
$$

Then $\mathscr{F} L$ is a 2 -sided ideal of the ring $L$. By a result of Jacobson, every subring $A$ of $L$ containing $L$ is a primitive ring $A$.

(c) Ring of linear transformations having adjoints. For $V$ any nondegenerate unitary space, the adjoint $\phi^{*}$ (if any) of $\phi \in L$ is defined to be the linear transformation $\phi^{*}$ of $V$ such that

$$
u \cdot v \varphi=u \phi \cdot v \quad(u, v \in V) .
$$

As is well-known, every linear transformation in $\mathscr{F} L$ has adjoint in $\mathscr{F} L$. Generally, if $\phi_{1}, \phi_{2} \in L$ have adjoints in $L$, then so must be $\phi_{1}^{*}, \phi_{1}+\phi_{2}$ and $\phi_{1} \phi_{2}$. Then:

$$
\begin{gathered}
\phi_{1}^{* *}=\phi_{1} \\
\left(\phi_{1}+\phi_{2}\right)^{*}=\phi_{1}^{*}+\phi_{2}^{*} \\
\left(\phi_{1} \phi_{2}\right)^{*}=\phi_{2}^{*} \phi_{1}^{*} .
\end{gathered}
$$


Hence, the set of linear transformations $\phi \in L$ having adjoints is a primitive subring of $L$. This ring is the domain of the partial operator $\phi \rightarrow \phi^{*}$. I refer to the partial mapping $\phi \rightarrow \phi^{*}$ as to a partial involution and to $(L ; *)$ as to partial involutorial ring.

(d) Unitary group $U-U(V)$. The partial involutorial ring $(L ; *)$ has a unitary group (general notation: $\mathscr{U}(L ; *)$ ), which is the group of elements $\phi \in L$ such that (i) $\phi$ is invertible (ii) $\phi^{*}$ is defined and (iii) $\phi^{*}=\phi^{-1}$. This group I will denote apart by $U=U(V)$.

(e) Center of $L$. Recall that $Z=$ center of $D$. Then $L$ can be turned into an algebra over $Z$, where the scalar multiplication $z \in Z, \phi \in L \rightarrow$ $z \phi \in L$ is:

$$
z \phi=v \rightarrow z(v \phi)
$$

The algebra $L$ is central in that $Z(L)=Z 1$, where $1=$ unity of $D$. Incidentally, if $z \in Z$, then taking $*$ in $D$ or $*$ in $L$ agree in that

$$
(z \cdot 1)^{*}=z^{*} \cdot 1 \text {. }
$$

Definition 2.1. Let $A$ be any central algebra over the valued field $(Z ; w)$. Assume that $A$ carries a partial involution $x \rightarrow x^{*}$ (partial antiautomorphism whose domain is a subring of $A$ such that $x^{* *}=x$ for every $x \in A$ and $\left.(z \cdot 1)^{*}=z^{*} \cdot 1\right)$. The binary relation $\mathscr{P}$ between $A$ and the group with infinity adjoined $G^{\#}$ is said to be a (*-prevaluation) (resp. strict $*$-prevaluation) if:

(i) For each pair $0 \neq z \in Z$ and $g \in G^{\#}, z \cdot 1 \mathscr{P} g \Leftrightarrow w(z) \geq g$ (resp. $w(z)>g)$.

(ii) For each triple $x \in A, g, g^{\prime} \in G^{\#}$, if $x \mathscr{P} g$ and $g \geq g^{\prime}$ then $x \mathscr{P} g^{\prime}$.

(iii) For each quadruple $x, x^{\prime} \in A, g, g^{\prime} \in G^{\#}$, if $x \mathscr{P} g$ and $x^{\prime} \mathscr{P} g^{\prime}$ then $x x^{\prime} \mathscr{P}\left(g+g^{\prime}\right)$.

(iv) For each quadruple as in (iii), $x+x^{\prime} \mathscr{P} \operatorname{Min}\left(g, g^{\prime}\right)$.

(v) For each pair $x, g \in A, G^{\#}$ with $x^{*}$ defined if $x \mathscr{P} g$ then $x * \mathscr{P} g$.

(vi) $x \mathscr{P} g$ for every $g \in G$ if, and only if, $x \mathscr{P} \infty$ if, and only if $x=0$.

The following examples will motivate Definition 2.1 .

ExAmple 2.2. Let $A$ be the $Z$-algebra $D$, relative to the ground involution $*$. Denote by $\cdots w \geq \cdots$ (resp. $\cdots \underline{w}>\cdots$ ) the binary relation between $D$ and $G^{\#}$ that is defined by

$$
\begin{gathered}
x \underline{w} \geq g \Leftrightarrow w(x) \geq g \\
\text { (resp. } x w>g \Leftrightarrow w(x)>g) .
\end{gathered}
$$

Then $\cdots \underline{w} \geq \cdots($ resp. $\cdots \underline{w}>\cdots)$ ) is a $*$-prevaluation (resp. a strict *-prevaluation). 
EXAMPLE 2.3. Let $D_{\mathscr{R}}$ be the division ring of right translations of $D$ viewed as an algebra over $Z$, relative to the involution

$$
x_{\mathscr{R}}^{*}=x_{\mathscr{R}}^{(s)},
$$

where $0 \neq s=s^{*}$ is fixed and $x^{(s)}=s x^{*} s^{-1}$. Denote by $\cdots w \geq \cdots$ (resp. $\cdots w>\cdots$ ) the binary relation between $D_{\mathscr{R}}$ and $G^{\#}$ that is defined by

$$
x_{\mathscr{R}} w \geq g \Leftrightarrow 2 w\left(u \cdot v x_{\mathscr{R}}\right) \geq w(u \cdot u)+w(v \cdot v)+2 g,
$$

for every pair $u, v$ in $D$, where $u \cdot v=u s v^{*}$.

$$
\text { (resp. } x_{\mathscr{R}} w>g \Leftrightarrow 2 w\left(u \cdot u v_{\mathscr{R}}\right)>w(u \cdot u)+w(v \cdot v)+2 g \text { ), }
$$

for every pair $u, v$ in $D$ such that $u \cdot u \neq 0$ and $v \cdot v \neq 0$ ). Then $\cdots w \geq$ $\cdots$ (resp. $\cdots w>\cdots)$ is a $*$-prevaluation (resp. a strict $*$-prevaluation).

Example 2.1 is formal. Example 2.2 reduces to the preceding example since, in effect, $x w \geq g \Leftrightarrow x_{\mathscr{R}} \underline{w} \geq g$ and $x_{\mathscr{R}} w>g \Leftrightarrow x \underline{w}>g$. Every 1-dimensional unitary space $V$, which is non-degenerate, can be identified to ${ }_{D} D$, relative to the coordinate form $u \cdot v=u s v^{*}$, where $0 \neq s$ is any element that is represented by the form in the sense $s=v \cdot v$, some $v \in V$. Then $L(V)=D_{\mathscr{R}}$ and the adjoint involution in $L$ is precisely the one in Example 2.2. By analogy with that example one is led to the

THEOREM 2.4. Let $V$ be any non-degenerate elliptic space and let $L$ be the ring of linear transformations of $V$ considered as an algebra over the center $Z$ of the ground division ring. Equip $L$ with the partial adjoint involution $\phi \rightarrow \phi^{*}$ and $Z$ with the ground valuation $w$. Then:

(1) The binary relation $\cdots w \geq \cdots$ between $L$ and $G^{\#}$ that is defined by

$$
\phi w \geq g \Leftrightarrow 2 w(u \cdot v \phi) \geq w(u \cdot u)+w(v \cdot v)+2 g,
$$

for every pair $u, v \in V$, is a $*$-prevaluation.

(2) The binary relation $\cdots w>\cdots$ between $L$ and $G^{\#}$ that is defined by

$$
\phi w>g \Leftrightarrow 2 w(u \cdot v \phi)>w(u \cdot u)+w(v \cdot v)+2 g ;
$$

for every pair $u, v \in V$ with $u \cdot u \neq 0, v \cdot v \neq 0$, is a strict $*$-prevaluation.

Theorem 2.4 is essentially evident. It is appropriate to add that the linear inequality in (12) and its strict version in (13) contain as particular cases the quadratic inequalities

$$
w(u \phi \cdot u \phi) \geq w(u \cdot u)+2 g,
$$


(15)

$$
w(u \phi \cdot u \phi)>w(u \cdot u)+2 g(u \cdot u \neq 0),
$$

respectively. Under the assumption $V$ is 0-ellitpic, (14) (resp. (15)) implies back

$$
\begin{aligned}
& 2 w(u \cdot v \phi) \geq w(u \cdot u)+w(v \cdot v)+2\left(g+\varepsilon_{0}\right) \\
&\left(\operatorname{resp.} 2 w(u \cdot v \phi)>w(u \cdot u)+w(v \cdot v)+2\left(g+\varepsilon_{0}\right),\right. \\
&u \cdot u \neq 0, v \cdot v \neq 0) .
\end{aligned}
$$

For future reference notice that (12) (resp. (13)) implies equally

$$
\begin{gathered}
w(u \cdot u \phi) \geq w(u \cdot u)+g \\
(\text { resp. } w(u \cdot u \phi)>w(u \cdot u)+g, u \cdot u \neq 0) .
\end{gathered}
$$

Definition 2.5. Let:

$$
\begin{gathered}
L_{g}=L_{g}(V ; w \geq)=\{\phi \in L \mid \phi w \geq g\} \quad\left(g \in G^{\#}\right) ; \\
L_{g}^{+}=L_{g}^{+}(V ; w \geq)=\{\phi \in L \mid \phi w>g\} \quad(g \in G) ; \\
L_{-\infty}=\bigcup_{g \in G} L_{g}=\left\{\phi \in L \exists g \in G^{\#} \mid \phi w \geq g\right\} .
\end{gathered}
$$

Refer to $L_{g}$ (resp. $L_{g}^{+}$) as to a congruence ideal if, further, $g \geq 0$.

The following results are, again, formal results.

$$
L_{g}\left(\text { resp. } L_{g}^{+}\right) \text {is an additive subgroup of } L,
$$

which is closed under $*$.

$$
\begin{gathered}
\left.g \geq g^{\prime} \Rightarrow L_{g} \subset L_{g^{\prime}}, \quad \text { (resp. } L_{g}^{+} \subset L_{g^{\prime}}^{+}\right) . \\
\bigcap_{g \in G} L_{g}=\bigcap_{g \in G} L_{g}^{+}=L_{\infty}=0 .
\end{gathered}
$$

$L_{-\infty}$ is a $*$-closed subring of $L$ containing $Z$ and evidently $L_{g}$ for every $g \in G$.

$$
\begin{gathered}
L_{g} \supset L_{g}^{+} \quad(g \in G) . \\
z L_{g}=L_{g+w(z)} \quad(z \in Z) . \\
z L_{g}^{+}=L_{g+w(z)}^{+} \quad(g \in G, 0 \neq z \in Z) . \\
L_{g}+L_{g^{\prime}} \subset L_{\operatorname{Min}\left(g, g^{\prime}\right)} . \\
L_{g} L_{g^{\prime}} \subset L_{g+g^{\prime}} \\
L_{g}+L_{g^{\prime}}^{+} \subset L_{\operatorname{Min}\left(g, g^{\prime}\right)}^{+} \quad\left(g \in G^{\#}, g^{\prime} \in G\right) .
\end{gathered}
$$




$$
\begin{array}{ll}
L_{g} L_{g^{\prime}}^{+} \subset L_{g+g^{\prime}}^{+} & \left(g \in G^{\#}, g^{\prime} \in G\right) . \\
L_{g}^{+} L_{g^{\prime}} \subset L_{g+g^{\prime}}^{+} & \left(g \in G, g^{\prime} \in G^{\#}\right) .
\end{array}
$$

For the rest of this section I will specialize to the congruence ideals $L_{g}$ or $L_{g}^{+}$, where $g \geq 0$. They are indeed 2-sided ideals of the ring $L_{0}$. By construction, the members $\phi \in L_{0}$ are the linear transformations $\phi$ such that

$$
2 w(u \cdot v \phi) \geq w(u \cdot u)+w(v \cdot v) .
$$

I refer to such a $\phi$ as to a linear transformation increasing lengths. Indeed as a special case of (15) one has the quadratic inequality

$$
w(u \varphi \cdot u \phi) \geq w(u \cdot u) \quad(u \in V) .
$$

In $L_{0}$ sits the 2-sided ideal $L_{0}^{+}$of linear transformations $\phi$ strictly increasing lengths since by construction $\phi \in L_{0}^{+}$if, and only, if,

$$
2 w(u \cdot v \phi)>w(u \cdot u)+w(v \cdot v)
$$

for every pair $u, v$ with $u \cdot u \neq 0, v \cdot v \neq 0$. Relationship between $L_{0}^{+} \subset$ $L_{0} \subset L_{-\infty}$ will follow. Let me first recapitulate some of the facts:

THEOREM 2.5. Let $V$ be any non-degenerate elliptic space. Then:

(1) The set $L_{0}$ of linear transformations $\phi$ that increase lengths

$$
2 w(u \cdot v \phi) \geq w(u \cdot u)+w(v \cdot v) \quad(u, v \in V),
$$

is a subrng of $L$, which is $*$-closed.

(2) In $L_{0}$ sits the 2-sided ideal of linear transformations $\phi$ that strictly increase lengths:

$$
2 w(u \cdot v \phi)>w(u \cdot u)+w(v \cdot v) \quad(u \cdot u \neq 0, v \cdot v \neq 0)
$$

(3) For each $g \geq 0, L_{g}\left(\right.$ resp. $\left.L_{g}^{+}\right)$is a 2-sided ideal of the rng $L_{0}$, which is contained in $L_{0}^{+}$, if $g>0$.

(4) If $L_{-\infty}=\bigcup_{g \in G} L_{g}$, then $L_{-\infty}$ is a $*$-closed subring of $L$ containing $Z$ and evidently $L_{0}$.

A key result for the considered treatment of the tower $L_{0}^{+} \subset L_{0} \subset L_{-\infty}$ will be the

THEOREM 2.6. Let $\left(V_{\iota}\right)_{\iota \in I}$ be any orthogonal decomposition of $V$ and suppose that $\left(g_{\imath}\right)$ is a family in the value group such that $g_{i} \geq g$ for every $\iota \in I$. If , further, $V$ is a non-degenerate $\varepsilon_{0}$-elliptic space then

$$
L_{g+2 \varepsilon_{0}}(V) \supset \bigoplus_{\iota \in I} L_{g_{\imath}}\left(V_{\iota}\right) \text {. }
$$


Proof. Let $\phi_{\iota} \cap L_{g_{\imath}}\left(V_{\imath}\right), \iota \in I$. Let $\phi=\bigoplus_{\iota \in I} \phi_{\imath}$. It is to be shown that $\phi \in L_{g+2 \varepsilon_{0}}(V)$. If $v \in V$ there is $\left(v_{\imath}\right)$ such that $v=\sum v_{i}$, where all $v_{\iota}=0$ but for finitely many indices. From $\phi_{\iota} \in L_{g_{\iota}}\left(V_{\iota}\right)$ follows $w\left(v_{\iota} \phi_{\iota} \cdot v_{\iota} \phi_{\iota}\right) \geq$ $w\left(v_{\imath} \cdot v_{\iota}\right)+2 g_{\imath}$. Then

$$
\begin{aligned}
w(v \boldsymbol{\phi} \cdot v \boldsymbol{\phi}) & =2\left(\sum v_{\iota} \phi_{\iota} \cdot v_{\iota} \phi_{\iota}\right) \geq \operatorname{Min}_{\iota \in I}\left(v_{\iota} \phi_{\iota} \cdot v_{\iota} \phi_{\iota}\right) \\
& \geq \operatorname{Min}_{\iota \in I}\left(w\left(v_{\iota} \cdot v_{\iota}\right)\right)+2 g_{\iota} \geq \operatorname{Min}_{\iota \in I}\left(w\left(v_{\iota} \cdot v_{\iota}\right)\right)-2 g .
\end{aligned}
$$

Since $\left(v_{\iota}\right)$ is an orthogonal family in $V$ and since $V$ is $\varepsilon_{0}$-elliptic it follows by a trivial adaptation of $\$ 1$, (29) (Theorem 1.7, implication 2. $\Rightarrow$ 3.) that

$$
\underset{g i \in I}{\operatorname{Min}}\left(w\left(v_{\iota} \cdot v_{\iota}\right)\right) \geq 2\left(\sum_{\iota \in I} v_{\imath} \cdot v_{\iota}\right)+2 \varepsilon_{0}
$$

so that

$$
w(v \phi \cdot v \phi) \geq w(v \cdot v)+2 \varepsilon_{0}+2 g,
$$

for every $v$ in $V$. From $\varepsilon_{0}$-ellipticity of $V$ follows $\phi \in L_{g+2 \varepsilon_{0}}(V)$, as desired.

THEOREM 2.7. If the rng $L_{0}$ has some one-sided unity then $L_{0}$ is a subring of L. Equivalently, $V$ is 0 -elliptic.

Proof. For let $\phi_{0}$ be any right unity of the $\operatorname{rng} L_{0}$. If $\phi$ is any projection (e.g. $\phi=\phi^{*}=\phi^{2} \in L$ ) with $V_{\phi}$ is finite-dimensional I assert that $\phi \phi_{0}=\phi$. For to begin with if $V^{(1)}=V \phi$ and $V^{(2)}=V(1-\phi)$ then $V=V^{(1)} \oplus V^{(2)}$. From Theorem 2.6 follows

$$
L_{0} \supset L_{-2 \varepsilon_{0}}\left(V^{(1)}\right) \oplus L_{-2 \varepsilon_{0}}\left(V^{(2)}\right) .
$$

Now $V^{(1)}$ has some orthogonal basis $f_{i}, i=1, \ldots, n$. Using this basis one can turn $V^{(1)}$ into a bi-space over $D$, relative to the right scalar multiplication

$$
v=\sum_{i=1}^{n} x_{i} f_{i}, \quad \lambda \in D \rightarrow v \lambda=\sum_{i=1}^{n} x_{i} \lambda f_{\iota} .
$$

Then $L\left(V^{(1)}\right)$ is a right vector space over $D$, relative to the scalar multiplication

$$
\phi \in L\left(V^{(1)}\right) \quad \lambda \in D \rightarrow \phi \cdot \lambda=v \rightarrow(v \phi) \lambda .
$$

If $\tilde{\lambda}=1_{V_{1}} \cdot \lambda$, then $\tilde{\lambda} \in L\left(V^{(1)}\right)$. I assert that in the ring $L\left(V^{(1)}\right)$

$$
\tilde{\lambda}_{w} \geq\left(2 \varepsilon_{0}+w(\lambda)\right)
$$


follows. For if $v \in V^{(1)}$, then

$$
v \tilde{\lambda} \cdot v \tilde{\lambda}=\sum_{j=1}^{n} \lambda_{j} \lambda s_{j}\left(\lambda_{j} \lambda\right)^{*} \quad\left(s_{j}=f_{j} \cdot f_{j}\right) .
$$

Thus

$$
w(v \tilde{\lambda} \cdot v \tilde{\lambda}) \geq 2 w(\lambda)+\operatorname{Min}\left(w\left(\lambda_{j} s_{J} \lambda_{J}^{4}\right)\right) \geq 2 w(\lambda)+2 \varepsilon_{0}+w(v \cdot v)
$$

giving

$$
2 w(u \cdot v \tilde{\lambda}) \geq w(u \cdot u)+w(v \cdot v)+2\left(2 \varepsilon_{0}+w(\lambda)\right),
$$

as desired.

Define $\lambda \phi$ to be the linear transformation on $V$

$$
v=v_{1} \oplus v_{2} \rightarrow v(\lambda \phi)=v_{1} \tilde{\lambda} \oplus 0 .
$$

For $\lambda \neq 0$ with $w(\lambda)$ large enough it is clear that $\lambda \phi \in L_{-2 \varepsilon_{0}}\left(V_{1}\right) \oplus$ $L_{-2 \varepsilon_{0}}\left(V_{2}\right)$ hence, $\lambda \phi \in L_{0}(V)$. Thus $(\lambda \phi) \phi_{0}=\lambda \phi$. Hence if $v=v_{1} \oplus v_{2}$, then

$$
v(\lambda \phi) \phi_{0}=\left(v_{1} \tilde{\lambda}\right) \phi_{0}=v_{1} \tilde{\lambda} .
$$

Since $\lambda$ is evidently an onto transformation of $V^{(1)}$ it follows that $v_{1} \phi_{0}=v_{1}$ for every $v_{1} \in V^{(1)}$ or, $\phi \phi_{1}=\phi$, for every projection $\phi$ of the space $V$ with $\phi$ of finite rank. Now, for every non-zero vector $u$ in $V$ if $\phi_{0}$ : $V \rightarrow V$ is defined by

$$
v \phi_{u}=(v \cdot u) \frac{1}{u \cdot u} u
$$

then as is well-known and easy, $\phi_{u}$ is a projection of rank 1. Hence, $\phi_{u} \phi_{0}=\phi_{u}$ or $u \phi_{0}=u$. As this holds for every $u, \phi_{0}=1$ follows; since $1 \in L_{0}, V$ is evidently 0 -elliptic.

THEOREM 2.7. Let $V$ be any non-degenerate elliptic space. Then:

(1) $L_{-\infty}$ is a primitive ring since, in fact, $L_{-\infty}$ contains all linear transformations of finite rank.

(2) If $V$ possesses some orthogonal basis then $L_{0}$ is an order in the ring $L_{-\infty}$ so that $L_{0}$ is a prime rng.

Proof. (1) (§3, Theorem 3.5, required). I will assume in what will follow that if $V$ is finite-dimensional then $L_{-\infty}(V)=L$. For general $V$ proceed as follows. Pick $\phi \in \mathscr{F} L$. Put: $\phi_{1}=\phi-\phi^{*}, \phi_{2}=\phi-\phi^{*}, V_{\iota}=$ $V_{\phi_{\iota}}, \iota=1,2$. Here, $V_{\iota}^{\perp}=\operatorname{Ker}\left(\phi_{\iota}\right)$ and since $V_{\iota}$ is finite-dimensional it follows that $V=V_{\iota} \oplus V_{\iota}^{\perp}$. If $\phi_{\iota 1}=\phi_{\iota} / V_{\iota}$ and $\phi_{\iota 2}=0_{V_{\iota}^{\perp}}$ then evidently 
$\phi_{\iota}=\phi_{\iota 1} \oplus \phi_{\iota 0}$. Since $V_{\iota}$ is finite-dimensional it follows that $\phi_{\iota_{1}} w \geq g$ for some $g \in G(i=1,2)$. By Theorem 2.5 follows $\phi_{\iota} \in L_{-\infty}$ so that $2 \phi=\phi_{1}$ $+\phi_{2} \in L_{-\infty}$. Since evidently $1 / 2 \in L_{-\infty}, \phi \in L_{-\infty}$ follows.

(2) Define a right scalar multiplication via the basis $\left(f_{\iota}\right)$ of $V$ by

$$
v=\sum x_{\iota} f_{\iota}, \quad \lambda \in D \rightarrow v \lambda=\sum_{\iota \in I} x_{\iota} \lambda f_{\iota} .
$$

Then define the right scalar multiplication

$$
\phi \in L \quad \lambda \in D \rightarrow \phi \lambda v \rightarrow(v \phi) \lambda .
$$

By a trivial adaptation of the calculation in Theorem 2.7 point 2 , follows that if $\tilde{\lambda}=v \rightarrow v \lambda$ then $\tilde{\lambda} w \geq\left(2 \varepsilon_{0}+w(\lambda)\right)$. Thus

$$
\tilde{\lambda} w \geq g \Rightarrow \phi \lambda w \geq\left(g+w(\lambda)+2 \varepsilon_{0}\right) .
$$

Given $\phi \in L_{-\infty}$ there is $g \in G$ such that $\phi w \geq g$. Then for $g^{\prime}$ large enough $g+g^{\prime}+2 \varepsilon_{0} \geq 0$. If $\lambda \in d$ is such that $w(\lambda)=g^{\prime} \neq \infty$ then $\lambda \neq 0$ and by the preceding $\phi \lambda \in L_{0}$. Then for $w(\lambda)$ large enough follows

$$
\phi=(\phi \lambda) \lambda^{-1}
$$

where both $\phi \lambda, \lambda \in L_{0}$. Hence $L_{0}$ is an order in $L_{-\infty}$ so that $L_{0}$ is a prime rng.

Definition 2.8. Call $\phi \in L$ medial or say $\phi$ preserve lengths, if

$$
w(v \phi \cdot v \phi)=w(v \cdot v)
$$

for every $v \in V$.

EXAMPLE 2.9. Let $V$ be the left space ${ }_{D} D$, relative to the coordinate form

$$
x \cdot y=x s y^{*} .
$$

To say that $\phi \in L$ is medial is to say that $\phi=x_{\mathscr{R}}$, where $x$ is a unit in the valuation ring $R$.

THEOREM 2.10. The set $\bar{M}=\bar{M}(V)$ of all medial transformations has the following features.

(1) For each $0 \neq z \in Z, z \cdot 1 \in \bar{M}$ if and only if $w(z)=0$.

(2) $\bar{M}$ is a multiplicative monoid consisting only of right invertible transformations $\phi$.

(3) $\bar{M}$ contains inverses (e.g. if $\phi \in \bar{M}$ and if $\phi$ is invertible in $L$ then $\left.\phi^{-1} \in M\right)$

(4) For each $\sigma \in L_{0}^{+}$, if $\phi=1+\sigma$, then $\phi \in \bar{M}$. 
(5) For each $g \in G, M L_{g} \subset L_{g}$ (resp. $M L_{g}^{+} \subset L_{g}^{+}$).

(6) If $\phi^{*}$ exists in $\bar{M}$ then $L_{g} \phi \subset L_{g}$ (resp. $L_{g}^{+} \subset L_{g}^{+}$).

(7) If $V$ is finite-dimensional then $\vec{M}$ is a group.

(8) If $V$ is 0-elliptic then the group of invertible elements in $\bar{M}$ is also the group of units of the ring $L_{0}$.

Proof. (1) To say that $z \cdot 1$ is medial is to say that $w(z v \cdot z v)=w(v \cdot v)$ for each $v \in V$; if, and only if, $2 w(z)=0$ or $w(z)=0$ (if $v \neq 0$ ).

(2) This is evident.

(3) This is, again, evident.

(4) From $\sigma \in L_{0}^{+}$follows for each $0 \neq v \in V$ :

$$
w(v \sigma \cdot v \sigma) £(v \cdot v) ; \quad w(v \sigma \cdot v)=w(v \cdot v \sigma)>w(v \cdot v) .
$$

By the strong triangle inequality follows

$$
w(v \cdot v \sigma)+v \sigma \cdot v+(v \sigma \cdot v \sigma)>w(v \cdot v),
$$

and by the special triangle equality follows

$$
w(v \cdot v+(v \cdot v \sigma+v \sigma \cdot v+v \sigma \cdot v \sigma))=w(v \cdot v)
$$

or,

$$
w(v(1+\sigma) \cdot v(1+\sigma))=w(v \sigma \cdot v \sigma)=w(v \cdot v),
$$

as desired.

(5) This is the same as showing that

$$
\tau w \geq g \Rightarrow \phi \tau w \geq g(\operatorname{resp} . \tau w>g \Rightarrow \phi \tau w>g)
$$

for every triple $\tau, \phi, g \in L, M, G$. For instance, if $\tau w>g$ then for every pair $u, v \neq 0$,

$$
2 w(u \cdot v(\phi \tau))=2 w(u \cdot(v \phi) \tau) .
$$

If $v \phi=0$ then $v \phi \tau=0$ so that

$$
2 w(u \cdot v(\phi \tau)) \geq w(u \cdot u)+w(v \cdot v)+2 g .
$$

If $v \phi \neq 0$ then,

$$
\begin{aligned}
2 w(u \cdot v(\phi \tau)) & =2 w(u \cdot(v \phi) \tau) \geq 2(u \cdot u)+w(v \phi \cdot v \phi)+2 g \\
& =w(u \cdot u)+w(v \cdot v)+2 g
\end{aligned}
$$

placing $\phi \tau$ in $L_{g}$.

(6) Again, if $\tau w>g$ and $\phi^{*} \in M$ then for $u \phi^{*} \neq 0$,

$$
\begin{aligned}
2 w(u \cdot v \tau \phi) & =2 w\left(u \phi^{*} \cdot v \tau\right)>w\left(u \phi^{*} \cdot u \phi^{*}\right)+w(v \cdot v)+2 g \\
& =w(u \cdot u)+w(v \cdot v)+2 g
\end{aligned}
$$


while $u \phi^{*}=0$ implies $2 w(u \cdot v \tau \phi)=2 w\left(u \phi^{*} \cdot v \tau\right)=\infty>w(u \cdot u)+$ $w(v \cdot v)+2 g$.

(7) Let $\phi$ be any invertible medial transformation. Then for every $v \in V$,

$$
w(v \phi \cdot v \phi)=w(v \cdot v) \geq w(v \cdot v) .
$$

Since $V$ is 0-elliptic it follows that

$$
\begin{aligned}
2 w(u \cdot v \phi) & \geq w(u \cdot u)+w(v \phi \cdot v \phi)=w(u \cdot u)+w(v \cdot v) \\
& \geq w(u \cdot u)+w(v \cdot v),
\end{aligned}
$$

placing $\phi$ in $L_{0}$. Since $\phi^{-1}$ is medial then, again, $\phi^{-1} \in L_{0}$. Hence $\phi$ is a unit of $L_{0}$. Conversely, let $\phi$ be a unit of the ring $L_{0}$. For each $v \in V$,

$$
\begin{aligned}
w(v \cdot v) & =w\left(\left(v \phi^{-1} j\right) \phi \cdot\left(v \phi^{-1}\right) \phi\right) \geq w\left(v \phi^{-1} \cdot v \phi^{-1}\right) \quad\left(\phi \in L_{0}\right) \\
& \geq w(v \cdot v) \quad\left(\phi^{-1} \in L_{0}\right) .
\end{aligned}
$$

Hence $w(v \cdot v)=w\left(v \phi^{-1} \cdot v \phi^{-1}\right)$. By symmetry, $w(v \cdot v)=w(v \phi \cdot v \phi)$. Hence $\phi$ and $\phi^{-1}$ are medial transformations, as desired.

The preceding theorem suggests that the medial transformations behave as potential units of the ring $L_{0}$ for, at least, the finite dimensional 0 -elliptic spaces $V$.

THEOREM 2.11. Let $V$ be any finite-dimensional non-isotropic unitary space over the involutorial division ring $D$ with characteristic $\neq 2$. Then:

(1) If $\phi \in L=L(V)$ has adjoint $\phi^{*}$ and if $\phi \phi^{*}=0$ then $\phi=0$.

(2) If $\phi=\phi^{*}$ is one-to-one then $\phi$ is invertible in $L$.

(3) If $\phi=-\phi^{*}$ then $1-\phi$ is invertible in $L$.

Proof. (1) and (2) are well-known and easy.

(3) Let $\phi$ be as in the statement. If $v \in V$ is such that $v \phi=v$ then $v=v \phi^{2}$ follows so that

$$
v \cdot v=v \phi \cdot v \phi=v \cdot(v \phi) \phi^{*}=v \cdot\left(-v \phi^{2}\right)=-v \cdot v
$$

giving $v \cdot v=0$ or $v=0$. This shows that $1-\phi$ is one-to-one. Repeating for $\phi$ replaced by $-\phi$ one gets that $1+\phi$ is one-to-one. Hence, $1-\phi^{2}=$ $(1-\phi)(1+\phi)$ is one-to-one. Since $1-\phi^{2}$ is symmetric it follows by 2 . that $1-\phi^{2}$ is invertible and, hence, $1-\phi$ is invertible.

THEOREM 2.12. Let $V$ be any finite-dimensional non-degenerate elliptic space. Then:

(1) The subset $M$ of medial transformations $\phi$ having medial adjoints $\phi^{*}$ is a group (medial group) such that $L_{g} \phi=\phi L_{g}=L_{g}$ (resp. $L_{g}^{+} \phi=\phi L_{g}^{+}=$ $L_{g}^{+}$) for every $\phi \in M$ and $g \in G$. 
(2) If $\mathscr{I}$ is any right ideal of $L_{0}$ which is contained in $L_{0}^{+}$then $\mathscr{I} \subset \operatorname{Jac}\left(L_{0}\right)$.

Proof. (1) Since both $\phi, \phi^{*}$ are medial and, hence, one-to-one, it follows that $\phi^{*} \phi$ is one-to-one. From Theorem 2.11 follows that $\phi^{*} \phi$ is invertible. Then $\phi$ is invertible. Similarly, $\phi^{*}$ is invertible. Also $\phi^{-1}$ is medial and has medial adjoint. The rest of the assertion follows readily from Theorem 2.10 .

(2) Let $\sigma \in \mathscr{I}$. Since $\sigma \in \mathscr{I} \subset L_{0}^{+}$it follows that $1+\sigma$ is medial. Since $\sigma^{*}$ is defined, and, hence, $\sigma^{*} \in L_{0}^{+}$it follows that $(1+\sigma)^{*}=1+\sigma^{*}$ is medial. Thus $1+\sigma \in M$ has inverse in $M$. If $1+\sigma^{\prime}$ is the inverse of $1+\sigma$ then

$$
-\sigma^{\prime}=\left(1+\sigma^{\prime}\right) \sigma \in\left(1+\sigma^{\prime}\right) L_{0}^{+} \subset L_{0}^{+} l .
$$

Hence $\sigma^{\prime} \in L_{0}^{+}$is a quasi-inverse of $\sigma$. As this holds for every $\sigma \in \mathscr{I}$ it follows that $\mathscr{I} \subset \operatorname{Jac}\left(L_{0}\right)$.

Definitions 2.13. (a) By congruence subgroup of the unitary group I mean any subgroup $N$ of $U$ of the form

$$
N=U \cap(1+\mathscr{I}),
$$

for some 2-sided ideal $\mathscr{I}_{\text {of }}$ the rng $L_{0}$.

(b) The congruence subgroup

$$
N^{\prime}=U \cap\left(1+L_{g}\right) \quad(g \geq 0)
$$

is denoted by $U_{g}$.

(c) The congruence subgroup

$$
N^{\prime \prime}=U \cap\left(1+L_{g}^{+}\right) \quad(g \geq 0)
$$

is denoted by $U_{g}^{+}$.

Two important congruence subgroups:

$$
\begin{aligned}
& U_{0}=\{\phi \in U \mid(\phi-1) w \geq 0\} ; \\
& U_{0}^{+}=\{\phi \in U \mid(\phi-1) w>0\} .
\end{aligned}
$$

Evidently, $U_{0}=U$ if, and only if, $v$ is 0 -elliptic. One can, of course, extend formulas (45), (46) for $g \leq 0$; but, there is only little gain to do so for what will follow here or elsewhere.

THEOREM 2.14. Let $V$ be any non-degenerate elliptic space. The congruence subgroups $U_{1}$ (resp. $\left.U_{g}^{+}\right), g \geq 0$, form a chain of normal subgroups of the unitary group $U$ such that:

(i) $g \geq g^{\prime} \Rightarrow U_{g} \subset U_{g}$, (resp. $U_{g}^{+} \subset U_{g^{\prime}}^{+}$; 
(ii) $\cap U_{g}=\cap U_{g}^{+}=(1)$;

(iii) $\left[U_{g^{\prime}}, U_{g^{\prime}}\right] \subset U_{g+g^{\prime}}\left(\right.$ resp. $\left.\left[U_{g^{\prime}}, U_{g^{\prime}}^{+}\right] \subset U_{g+g^{\prime}}^{+}\right)$.

Proof. (0) $U_{g}$ (resp. $U_{g}^{+}$) is a normal subgroup of $U$. For let $\phi \in U_{g}$. Then $\phi-1 \in L_{g} \subset L_{0}$. Since $\phi$ has adjoint it follows that $\phi^{*}-1 \in L_{g}$ and since $\phi^{*}=\phi^{-1}, \phi^{-1} \in U_{g}$ follows. Let $\phi, \sigma \in U_{g}$. Then

$$
1-\phi \sigma=\phi(1-\sigma)+(1-\phi) \in \phi L_{g}+L_{g} .
$$

Since $\phi \in U \subset M$, it follows that $L_{g} \subset L$ (Theorem 2.12, point (1)) so that $1-\phi \sigma \in L_{g}$. Thus $\phi \sigma \in U_{g}$. Let $\tau \in U$. Then $\tau L_{g} \tau^{-1}=L_{g}$ (Theorem 2.12, point 1.). Hence $\tau U_{g} \tau^{-1}=U_{g}$ and $U_{g} \triangleleft U$. For the assertion $U_{g}^{+} \triangleleft U$ use similar argument.

(i), (ii) This is evident.

(iii) Let me show, say, that $\phi \in U_{g^{*}}, \tau \in U_{g}^{+}$, together imply $[\phi, \tau]$ $\left(=\phi^{-1} \tau^{-1} \phi \tau\right) \in U_{g+g^{*}}^{+}$. Indeed,

$$
\begin{aligned}
{[\phi, \tau] } & =\phi^{-1} \tau^{-1}(\phi \tau-\tau \phi) \\
& =\phi^{-1} \tau^{-1}((\phi-1)(\tau-1)-(\tau-1)(\phi-1)) \\
& \in \phi^{-1} \tau^{-1}\left(L_{g} L_{g^{*}}^{+} L_{g}\right) \\
& \subset \phi^{-1} \tau^{-1} L_{g+g^{*}}^{+} \subset L_{g+g^{*}}^{+} .
\end{aligned}
$$

THEOREM 2.15. (i) Each congruence subgroup $U_{g}^{+}$with $g \geq w(2)$, consists only of unitary transformations $\phi$ that can be Cayley parametrised.

(ii) Hence no $\phi \in U_{g}^{+}$can reverse $0 \neq v \in V$ and, consequently, $U_{g}^{+}$ excludes any involution $\sigma \neq 1$.

Proof. (i) It suffices to establish the theorem for $g=w(2)$. Given $\phi \in U_{w(2)}^{+}$and $v \in V$ if $v(1+\phi)=0$ but, $v \neq 0$ then since $\phi-1 \in L_{w(2)}^{+}$,

$$
w(v(\phi-1) \cdot v(\phi-1))>w(v \cdot v)+2 w(2),
$$

follows; but, the left member of this inequality is precisely $w(2 v \cdot 2 v)=$ $w(v \cdot v)+2 w(2)$. This shows that $1+\phi$ is one-to-one. Again $\phi^{*} \in U_{w(2)}^{+}$ forces $1+\varphi^{*}$ is one-to-one. By Theorem 2.12, $1+\phi$ is then invertible. Let $\tau=(1-\phi) /(1+\phi)$. By construction, $\tau^{*}$ is defined and $\tau^{*}=-\tau$. Here, $1+\tau$ is invertible as this follows from Theorem 2.12. Moreover, $\phi=(1-\tau) /(1+\tau)$, the usual Cayley parametrisation of the unitary $\phi$. (ii) This is partly shown in (i) and partly evident.

The following theorem is technical and will be used here and elsewhere. 
TheOReM 2.16. Let $V$ be any non-degenerate elliptic space. Let $\mathscr{C}$ be the partial operator of the ring $L$ which is defined at $\phi \in L$ if and only if $1+\phi$ is invertible and then

$$
\mathscr{C}(\phi)=2 \frac{1-\phi}{1+\phi} .
$$

If $\mathscr{C}^{-1}$ is the partial operator of $L$ which is defined at $\tau \in L$ if and only if $1+\tau / 2$ is invertible and then

$$
\mathscr{C}^{-1}(\tau)=\frac{1-\tau / 2}{1+\tau / 2}
$$

then:

(1) $\mathscr{C}\left(\mathscr{C}^{-1} \mid \tau\right)=\tau$ and $\mathscr{C}^{-1}(\mathscr{C}(\phi))=\phi$ for every pair $\tau$, $\phi$ with $\mathscr{C}^{-1}$ and $\mathscr{C}(\tau)$ defined.

(2) $\mathscr{C}\left(\right.$ resp. $\left.\mathscr{C}^{-1}\right)$ is one-to-one.

(3) $\mathscr{C}$ is entirely defined at $U_{g}$ (resp. $U_{g}^{+}$) for every $q>w(2)$ (resp. $g \geq w(2))$ and

$$
\mathscr{C}\left(U_{g}\right)=k_{g}(V)\left(\text { resp. }\left(U_{g}^{+}\right)=k_{g}^{+}\right)
$$

where $k_{g}(V)=\left\{\tau=-\tau^{*} \in L \mid \tau w \geq g\right\}$ provided $V$ is finite dimensional.

(4) $\mathscr{C}^{-1}$ is entirely defined at $k_{g}\left(\right.$ resp. $\left.k_{g}^{+}\right)$and

$$
\mathscr{C}^{-1}\left(k_{g}\right)=U_{g}\left(\text { resp. } \mathscr{C}^{-1}\left(k_{g}^{+}\right)=U_{g}^{+}\right),
$$

where $g \geq w(2)$ (resp. $g>w(2))$ provided $V$ is finite dimensional.

Proof. (1) and (2) are formal.

(3) and (4) If $g \geq w(2)$ then for each $\phi \in U_{g}^{+}$it is clear that $\phi \in U_{w(2)}^{+}$. From Theorem 2.15 follows that $1+\phi$ is invertible. Thus $\mathscr{C}(\phi)$ is defined. If

$$
\tau=\mathscr{C}(\phi)=2 \frac{1-\phi}{1+\phi}
$$

then by construction $\tau^{*}=-\tau$. Also,

$$
\tau=2 \frac{1-\phi}{2+(\phi-1)}=\frac{1-\phi}{1+((\phi-1) / 2)} .
$$

From $\phi \in U_{g}^{+}$follows $(\phi-1) \in L_{g}^{+}$so that $(\phi-1) / 2 \in L_{q-w(2)}^{+} \subset L_{0}^{+}$. Thus $1+(\phi-1) / 2 \in M$ so that

$$
\tau \in M L_{g}^{+}=L_{g}^{+}
$$

and, hence, $\tau \in k_{g}^{+}$.

Similarly if $g>w(2)$ then for each $\phi \in U_{g}, \tau=\mathscr{C}(\phi)$ is defined and $\tau \in k_{g}$. 
Conversely, if $\tau \in \mathfrak{k}_{g}^{+}$where $g \geq w(2)$ then evidently $\mathscr{C}^{-1}$ is defined at $\tau / 2$. (In fact, $1+\tau$ is invertible for every $\tau=-\tau^{*}$ ). If $\phi=\mathscr{C}^{-1}(\tau)$ then by construction $\phi$ is unitary and

$$
\phi-1=\frac{1-\tau / 2}{1+\tau / 2}-1=\frac{-\tau}{1+\tau / 2} \in M k_{g}^{+} \subset M L_{g}^{+}=L_{g}^{+} .
$$

Thus $\phi \in U_{g}$. Similarly if $\tau \in \mathscr{k}_{g}$ where $g>w(2)$ then $\tau \cdot \phi=\mathscr{C}^{-1}(\tau) \in$ $U_{g}$.

THEOREM 2.17. Let $V$ be any non-degenerate elliptic space, which is not the 1-dimensional orthogonal space. For every $g \in G$, the congruence subgroup $U_{g}$ (resp. $\left.U_{g}^{+}\right)$is not the identity subgroup.

Proof. By Theorem 2.16 it suffices to show that $k_{g}(V) \neq 0$ (resp. $k_{g}^{+}(V) \neq 0$ ). As a second reduction, since $k_{g}^{+} \subset k_{g}$ it suffices to show that $k_{g}^{+} \neq 0$. As a third reduction, still, since from a trivial adaptation of Theorem 2.6, follows that if $V^{(1)}$ is any orthogonal summand of $V$ then

$$
k_{g}^{+}(V) \supset k_{g-2 \varepsilon_{0}}\left(V^{(1)}\right) \oplus 0,
$$

one may assume, further, that $V$ is finite dimensional. By the same token as soon as a given subspace $V^{(2)}$ of $V$ verifies the theorem so will be all of $V$. If then $V$ is not orthogonal, then picking any 1-dimensional subspace $V^{(3)}$ of $V$ and observing that $k_{g}^{+}\left(V^{(3)}\right) \neq 0$ the theorem follows. If, on the other hand, $V$ is an orthogonal space one can quote Artin's [1, Theorem 5.7]. To motivate the reader, let me give a direct proof. From finite-dimensionality of $V$ follows that $L_{-\infty}(V)=L$. Pick any $0 \neq \tau=-\tau^{*}$ (possible since $\operatorname{dim}_{D} V \geq 2$ ). Then $\tau w \geq g$ for some $g_{0}$. Choose any $0 \neq z \in Z=D$ with $w(z)$ large enough so as $w(z)+g_{0}>g$. Then $z \tau \in L_{g_{0} w(z)}^{+} \subset L_{g}^{+}$. By construction, $0 \neq z \tau=-(z \tau)^{*}$.

THEOREM 2.18. Let $V$ be any non-isotropic unitary space over the *-valued division ring $(D ; * ; w)$, which is finite dimensional but other than the 1-dimensional orthogonal space. The following requirements are equivalent.

(1) There is $0 \neq v \in V$ and $g \in G$ such that if $u \in V$ then $2 w(v \cdot v) \geq$ $w(u \cdot u)+2 g$.

(2) There is some $0 \neq \phi \in L$ and $g \in G$ such that $\phi w \geq g$.

(3) $U_{g} \neq 1$ for some $g \in G$.

(4) $V$ is elliptic.

Proof. By Theorem 2.17, (4) $\Rightarrow$ (3). Evidently, (3) $\Rightarrow(2) \Rightarrow$ (1). Assume (1). Quoting [2, Theorem 1] or directly one can show that $U$ acts 
irreducibly on $V$. Hence there is a basis of $V$ of the form $v_{i}=v \phi_{l}$, where $\phi_{1}=1, \phi_{2}, \ldots, \phi_{n} \in U$. Given $u \in V$ there is a unique row vector $\underline{u}=$ $\left(x_{1}, \ldots, x_{n}\right)$ over $D$ such that $u=\sum x_{i} v_{i}$. I proceed to establish that, if $u_{1}, u_{2} \in V$ then

$$
2 w\left(u_{1} \cdot u_{2}\right) \geq w\left(u_{1} \cdot u_{1}\right)+2 \underline{w}\left(\underline{u}_{2}\right)+2 g,
$$

where $\underline{w}\left(x_{1}, \ldots, x_{n}\right)=\operatorname{Min}_{i=1, \ldots, n}\left(w\left(x_{i}\right)\right)$. For let $\underline{u}_{2}=\left(x_{1}, \ldots, x_{n}\right)$. Then

$$
\begin{aligned}
& 2 w\left(u_{1} \cdot x_{i} v_{i}\right)=2 w\left(u_{1} \cdot x_{i} v \phi_{i}\right)=2 w\left(u_{1} \phi_{i} \cdot x_{i} v\right) \\
&=2 w\left(u_{1} \phi_{i} \cdot v\right)+2 w\left(x_{i}\right) \geq w\left(u_{1} \phi_{i} \cdot u_{1} \phi_{i}\right)+2 w\left(x_{i}\right)+2 g \\
&=w\left(u_{1} \cdot u_{1}\right)+2 w\left(x_{i}\right)+2 g \geq w\left(u_{1} \cdot u_{1}\right)+2\left(\underline{w}\left(\underline{u}_{2}\right)+g\right) ; \\
& 2 w\left(u_{1} \cdot u_{2}\right)= 2 w\left(u_{1} \cdot \sum x_{i} v_{i}\right) \geq 2 \operatorname{Min}_{i}\left(w\left(u_{1} \cdot x_{i} v_{i}\right)\right) \\
& \geq w\left(u_{1} \cdot u_{1}\right)+2\left(\underline{w}\left(\underline{u}_{2}\right)+g\right),
\end{aligned}
$$

as desired. It remains then to show that for some $g^{\prime} \in G, 2 \underline{w}(\underline{u}) \geq$ $w(u \cdot u)+2 g^{\prime}$, for every $u \in V$. This can be readily seen from the observations:

$$
\underline{u}=\left(x_{1}, \ldots, x_{n}\right)=\left(u \cdot v_{1}, \ldots, u \cdot v_{n}\right) T,
$$

where $T=\left(S^{\text {tr.** }}\right)^{-1}$;

$$
\underline{w}(\underline{u}) \geq \underline{w}\left(u \cdot v_{1}, \ldots, u \cdot v_{n}\right)+\underline{w}(T),
$$

where $\underline{w}(T)$ is the minimum value of the entries in $T$.

As stated in Introduction, Theorem 2.18 is due to Artin in the orthogonal case and the proof is inspired by Artin's. The last item for this section will be the treatment of roots of unity in the congruence subgroup $L_{0}^{+}$.

THEOREM 2.19. Let $V$ be any non-degenerate elliptic space and let $P$ be the prime subfield of the ground division ring $D$. Then:

(1) If the valuation $w$ induces the trivial valuation on $P$, then $U_{0}^{+}$is torsion-free.

(2) If $w$ induces a non-trivial valuation on $P$ then if $p=\operatorname{charac}(\bar{D})$ then $U_{w(p)}^{+}$is torsion-free.

Proof. (1) It is to be shown first that if $\phi \in L_{0}^{+}$is algebraic over $P$ then, in fact, $\phi$ is nilpotent. For then there is a polynomial $p(t)$ over $P$ without constant term such that $\phi^{n}=p(\phi) \phi^{2 n}$ for some $n$. If $\tau=p(\phi) \phi^{n}$ then $\tau$ is an idempotent transformation. Since $w\left(P^{x}\right)=w\{r \mid 0 \neq r \in P\}$ is evidently contained in the medial group $M$ it follows that $\tau \in L_{0}^{+}$and, 
hence, $\tau$ strictly decreases lengths. If now $v \tau \neq 0$ for some $v$ it would follow

$$
w(v \tau \cdot v \tau)=w\left(v \tau^{2} \cdot v \tau^{2}\right)>w(v \tau \cdot v \tau),
$$

a contradiction. This shows that $V \tau=0$ so that $\tau=0$ and, hence, $\phi^{n}=0$.

Let then $\sigma \in U_{0}^{+}$be any root of unity. If $\phi=\sigma-1$ then by construction $\phi \in L_{0}^{+}$is algebraic over $P$. By the preceding $\phi$ is nilpotent. Now evidently $w(2)=0$ so that $L_{0}^{+}=L_{w(2)}^{+}$and, hence, $\sigma$ is Cayley parametrisable. If $\sigma=(1-\tau) /(1+\tau)$ with $\tau=-\tau^{*}$ then $\tau=(1-\sigma) /(1+\sigma)$ is nilpotent. In view of Theorem 2.11, $\tau=0$ or $\sigma=1$, as desired.

(2) To say that $w / p$ is not trivial is to say that $\operatorname{charac}(\bar{D})=p \neq 0$ (where $\bar{D}=R / J$ ) but, $\operatorname{charac}(D)=0$ so that $w(p)$ is a well-defined element in $G$.

Claim 1. Let $1 \neq \phi \in U_{0}^{+}$be any root of unity. The multiplicative order of $\phi$ is a power of $p$.

For let $m$ be the multiplicative order of $\phi$. Let $p^{r}$ be the highest power of $p$ dividing $m$. Then $m=p^{r} l$, where $p+l$. Suppose that $l \neq 1$. If $\sigma=\phi^{p^{r}}$, then $\sigma \neq 1$ and $\sigma$ has order $l$. Moreover, $\sigma \in U_{0}^{+}$for $\sigma$ is a power of $\phi \in U_{0}^{+}$. Let $\tau=\sigma-1$. From the relation $\sigma^{l}=1$ follows $((\sigma-1)+1)^{l}$ $=(\tau+1)^{l}=1$. Thus there are integers $r_{2}, r_{3}, \ldots, r_{l}$ such that

$$
l \tau+\sum_{i=2}^{l} r_{i} \tau^{i}=0
$$

Dividing through the preceding equation by $l$ this gives after factorisation by $\tau$

$$
\tau\left(1+\sum_{i=2}^{l} r_{l}^{\prime} \tau^{i-1}\right)=0 \quad\left(r_{i}^{\prime}=r_{i} / l\right) .
$$

Now, from $\sigma \in U_{0}^{+}$follows $\tau=\sigma-1 \in L_{0}^{+}$. Hence $\tau^{i-1} \in L_{0}^{+}(i \geq 2)$. Also,

$$
r_{i}^{\prime} \tau^{i-1} \in r_{i}^{\prime} L_{0}^{+} \in \frac{1}{l} r_{i} L_{0}^{+} \subset \frac{1}{l} L_{0}^{+}=L_{w(1 / l)}^{+}=L_{0}^{+} .
$$

Hence, $\sum_{i \geq 2}^{l} r_{i} \tau^{i-1} \in L_{0}^{+}$. From this, if $\tau_{0}=\sum_{i \geq 2}^{l} r_{i} \tau^{i-1}$, then $1+\tau_{0}$ is medial. By the basic equation, $\tau\left(1+\tau_{0}\right)=0$. Now $1+\tau$ is one-to-one and $\tau$ commutes with $1+\tau_{0}$. Hence $\tau=0$ or, $\sigma=1$, a contradiction. This shows that the order $m$ of $\phi$ is a power of $p$, as desired. 
Claim 2. If $w / P$ is the 2-adic valuation then $U_{w(2)}^{+}$is torsion-free.

Deny this claim. Pick $1 \neq \phi \in U_{w(2)}^{+}$and let $m \neq 1$ be the multiplicative order of $\phi$. By the preceding claim, $m=2^{r}$ for some integer $r \neq 0$. If $\phi_{1}=\phi^{2^{r-1}}$ then $\phi_{1} \neq 1$. Hence, $\phi_{1}$ is an involution belonging evidently to $U_{w(2)}^{+}$, contradicting Theorem 2.15 .

Claim 3. If $w / p$ is the $p$-adic valuation with $p$ odd then $U_{w(p)}$ is torsion-free so that $U_{w(p)}^{+}$is torsion-free.

Deny this claim. There is $1 \neq \phi \in U_{w(p)}$ with multiplicative order $p$. If $\tau=\phi-1$ and if the $\left(\begin{array}{l}p \\ l\end{array}\right)$ are the usual binomial coefficients then from $\phi^{p}=1$ follows

$$
p \tau+\left(\begin{array}{l}
p \\
2
\end{array}\right) \tau^{2}+\cdots+\left(\begin{array}{l}
p \\
p
\end{array}\right) \tau^{p}=0
$$

Since $p$ is prime, $p \mid\left(\begin{array}{l}p \\ l\end{array}\right)$ follows for $i=2, \ldots, p-1$. Dividing through by $p$ and isolating the last term this gives

$$
\frac{1}{p} \tau^{p}=\tau\left(1+\tau_{0}\right)
$$

where $\tau_{0}=-\left(r_{2} \tau+\cdots+r_{p-1} \tau^{p-2}\right)$ and $r_{2}, \ldots, r_{p-1}$ are integers. Now $w(p)>0$ for $w(p) \geq 0$ and $w / P$ is $p$-adic. Hence, $U_{w(p)} \subset U_{0}^{+}$. Thus, $\tau \in L_{0}^{+}$. From this $\tau_{0} \in L_{0}^{+}$so that $1+\tau_{0}$ is medial. In terms of lengths the factor $1+\tau_{0}$ can be thus neglected, that is,

$$
w\left(v\left(\tau\left(1+\tau_{0}\right)\right) \cdot v\left(\tau\left(1+\tau_{0}\right)\right)\right)=w(v \tau \cdot v \partial t) .
$$

Since $(1 / p) \tau^{p}=\tau\left(1+\tau_{0}\right)$ it follows that

$$
w\left(v \frac{1}{p} \tau^{p} \cdot v \frac{1}{p} \tau^{p}\right)=w(v \tau \cdot v \tau)
$$

or,

$$
w\left(v \tau^{p} \cdot v \tau^{p}\right)=w(v \tau \cdot v \tau)+2 w(p) .
$$

Restricting the preceding equality to $V^{(1)}=V \tau$ this gives

$$
w\left(v \tau^{p-1} \cdot v \tau^{p-1}\right)=w(v \cdot v)+2 w(p),
$$

for every $v \in V^{(1)}$. Now, the restriction $\tau^{(1)}$ of $\tau$ to $V^{(1)}$ is evidently a linear transformation of $V^{(1)}$. From $\tau \in L_{w(p)}$ follows $\tau^{(1)} \in L_{w(p)}\left(V^{(1)}\right)$. Hence $\left(\tau^{(1)}\right)^{p-1} \in L_{(p-1) w(p)}\left(V^{(1)}\right)$ (Equation (33)). Thus

$$
w\left(v \tau^{p-1} \cdot v \tau^{p-1}\right) \geq w(v \cdot v)+2(p-1) w(p)
$$


for every $v \in V^{(1)}$. Hence,

$$
w(v \cdot v)+2 w(p) \geq w(v \cdot v)+2(p-1) w(p) .
$$

If now $v \neq 0, v \in V^{(1)}$, this gives

$$
2 w(p) \geq 2(p-1) w(p) ; 2(p) \geq(p-1) w(p) ;(p-2) w(p) \leq 0 .
$$

However $p$ is an odd prime. Thus $p-2$ is a non-zero natural number. In view of the relation $w(p)>0,(p-2) w(p)>0$ follows, a contradiction. This shows that $V^{(1)}=0$ or $V \tau=0$, that is, $\tau=0$ or, $\phi=\tau+1=1$, a contradiction.

Question (open). Must $U_{0}^{+}$be always torsion-free?

To conclude this section, let me make two side remarks. The information about the normal subgroup structure of the unitary group as obtained in Theorem 2.14 can be carried over (almost verbatim) to the normal subgroup structure of the medial group $M$ (that is, the group of units of the medial semi-group $\bar{M}$ which have adjoints). The only difference arises in the fact that while $\sigma-1$ nilpotent with $\sigma \in U_{w(2)}^{+}$implies $\sigma=1$, one cannot decide as neatly in the case $\sigma \in M_{w(2)}^{+}$. As a result, in the preceding theorem, roots of unity in $M_{0}^{+}$can occur when $\operatorname{charac}(D)=p \neq 0$; these are, of course, $p$ roots of unity. If $\operatorname{charac}(\bar{D})=p$ and $\operatorname{charac}(D)=0$ then $M_{w(p)}^{+}$is torsion-free. The second remark is of different nature. I stated in the Introduction that my motivation in the ring $L_{0}$ arises equally from the ring $A$ that is spanned by $U$. In a continuation of this work I will show to what extent $A$ coincides with $L_{0}$ and/or $L_{0}$ and $A$ have essentially the same ideal structure.

3. Bounded orthogonal bases. In this section, the following situation will be analysed: Relative to a given orthogonal basis $\left(f_{i}\right)$ the non-isotropic space $V$ is such that for each unitary transformation $\phi$ the matrix of $\phi$ is with entries from a fixed additive subgroup $J_{g}$ of the valued division ring $D$. Special attention is given to the case $g=0$. In that case and assuming that $1 / 2 \in R$ the results will be quite complete. I begin with notations to be used throughout this section.

(a) Row finite matrix $\phi$. All matrices $\Phi$ are row-finite matrices. However, the number of rows of $\Phi$ is any cardinal number. Thus $\Phi=$ $\left[\phi_{i j}\right]_{i, j \in I}$ is such that:

$$
\phi_{i j}=\operatorname{entr}_{i, j}(\Phi) \in D ;
$$

$$
\text { For each } i \in I,\left(\phi_{j}\right)_{j \in I} \text { is a nullary row vector over } D \text {. }
$$


(b) Matrix of inner products $S$. Let $\left(f_{i}\right)_{i \in I}$ be a fixed orthogonal basis of $V$. I will consistently denote the inner product $f_{i} \cdot f_{i}$ by $s_{i}$. Thus the matrix of the inner products $f_{i} \cdot f_{j}$ is the diagonal matrix

$$
S=\operatorname{Diag}\left\{s_{i}\right\}_{i \in I} \quad\left(s_{i}=f_{i} \cdot f_{i}\right)
$$

(c) Matrix involutions. The standard matrix involution tr.* is the partial involution of the ring $D_{I \times I}$ of all $I \times I$ row-finite matrices $\Phi=$ $\left[\phi_{i j}\right]_{i, j \in I}$ over $D$, which is defined at $\Phi$ if, and only if, $\Phi^{\text {tr. }} \in D_{I \times I}$. Then

$$
\Phi^{\operatorname{tr} . *}=\left[\phi_{j i}^{*}\right]_{i, j \in I}
$$

The matrix of inner products $S=\operatorname{Diag}\left\{s_{\imath}\right\}_{\iota \in I}$ induces a new partial involution $(S)$ of $D_{I \times I}$ which is defined exactly when tr.* is defined and then

$$
\Phi^{(S)}=S \Phi^{\text {tr.**}} S^{-1}
$$

Explicitly,

$$
\operatorname{entr}_{i, j}\left(\Phi^{(S)}\right)=s_{i} \operatorname{entr}_{j, i}(\Phi)^{*} s_{j}^{-1} \quad(i, j \in I) .
$$

(d) Matrix prevaluation. Let $\cdots \underline{w} \geq \cdots$ be the binary relation between the $Z$-algebra $D_{I \times I}$ and the group with infinity adjoined $G^{\#}$, which is defined by

$$
\begin{aligned}
\Phi \underline{w} \geq g \Leftrightarrow \operatorname{entr}_{i, j}(\Phi) \underline{w} \geq g(\text { all } i, j \in I) \\
\quad\left(\text { e.g. } w\left(\operatorname{entr}_{i, j}(\Phi)\right) \geq g, \text { all } i, j \in I\right) .
\end{aligned}
$$

Then $\cdots \underline{w} \geq \cdots$ is a $*$-prevaluation relative to the involution tr.*; but, $\cdots \underline{w} \geq \cdots$ is generally not a $*$-prevaluation relative to the matrix involution $(S)$. Hence it is better to refer to this case as matrix prevaluation.

(e) Bounded basis (f). I shall say that the orthogonal basis $(f)$ is bounded if there is $g_{0} \in G$, which I call a bound for $\left(f_{i}\right)$, such that

$$
w\left(s_{\iota}\right)-w\left(s_{j}\right) \leq g_{0}
$$

for every pair $i, j \in I$ - Recall that $s=f \cdot f$. Evidently every finite-dimensional unitary space $V$ over the *-valued division ring $D$ has all its orthogonal bases bounded, the case $\varepsilon$ is the least bound for the given basis $(f)$ is noteworthy but not required in what will follow (unless otherwise specified).

(f) Matrix of a linear transformation. Throughout the rest of this section $V$ stands for any non-isotropic unitary space over the *-valued division ring. I will assume, further, that $V$ is equipped with the fixed 
orthogonal basis $\left(f_{i}\right)$. To the vector $v \in V$ associate the nullary row vector

$$
\underline{v}=\left(x_{i}\right) \Leftrightarrow v=\sum_{i \in I} x_{\iota} f_{i} .
$$

To $\phi \in L$ associate the matrix of $\phi, \operatorname{Mtrx}(\phi)$ (or $\underline{\phi}) \in D_{I \times I}$ determined by the equations

$$
\operatorname{Row}_{i}(\operatorname{Mtrx}(\phi))=\left(\phi_{i j}\right)_{j \in I} \Leftrightarrow f_{i} \phi=\left(\phi_{i j}\right)_{j \in I} \quad(\iota \in I) .
$$

Definition 3.1. I will call the basis $\left(f_{t}\right) \varepsilon_{0}^{\prime}$-elliptic, where $\varepsilon_{0}^{\prime} \in G$ is fixed, if

$$
\text { For each } \phi \in U=U(V), \operatorname{Mtrx}(\phi) \underline{w} \geq \varepsilon_{0}^{\prime} .
$$

THEOREM 3.2. Let $V$ be any non-isotropic unitary space and let $\left(f_{i}\right)$ be any orthogonal basis of $V$. The following requirements are equivalent.

(1) $\left(f_{i}\right)$ is an elliptic basis $\left(\right.$ e.g. $\left(f_{i}\right)_{i \in I}$ is $\varepsilon_{0}^{\prime}$-elliptic for some $\left.\varepsilon_{0}^{\prime} \in G\right)$

(2) $V$ is an elliptic space and $\left(f_{l}\right)$ is bounded.

$$
\operatorname{Proof}(1) \Rightarrow(2)
$$

Claim 1. There is $\varepsilon_{0} \in G$ such that if $\left(x_{i}\right)_{i \in I}$ is any nullary row vector over $D$, then

$$
\operatorname{Min}_{i \in I} w\left(x_{i} s_{i} x_{i}^{*}\right) \geq w\left(\sum_{j \in I} x_{j} s_{j} x_{j}^{*}\right)+\varepsilon_{0} .
$$

If $\left(x_{i}\right)=0$ there is nothing more to show. If $\left(x_{i}\right) \neq 0$ then if $v=\sum x_{i} f_{i}$ then $v \neq 0$ so that $v \cdot v \neq 0$. Hence, the linear transformation $\sigma=\sigma_{v}$ defined by

$$
u \sigma=u \cdot v \frac{1}{v \cdot v} v
$$

is a projection of $V$ (that is, $\sigma^{2}=\sigma=\sigma^{*}$ ). Thus $\phi=1-2 \sigma$ is a unitary transformation of $V$. Let then $\varepsilon_{0}^{\prime}$ be such that $\left(f_{i}\right)$ is $\varepsilon_{0}^{\prime}$-elliptic. By definition, if $\Phi$ is the matrix $\phi$ then $\Phi \underline{w} \geq \varepsilon_{0}^{\prime}$. Now, $\Phi=1-2 \operatorname{Mtrx}(\sigma)$. From this follows

$$
\operatorname{entr}_{i, j}(\underline{\sigma}) \geq \varepsilon_{0}^{\prime}-w(2) \quad(i, j \in I),
$$

where $\underline{\sigma}=\operatorname{Mtrx}(\sigma)$.

A simple calculation shows that

$$
\operatorname{entr}_{i, j}(\underline{\sigma})=\left(f_{i} \cdot x_{i} f_{i}\right) \frac{1}{v \cdot v} x_{j}
$$


If $\varepsilon_{0}=\varepsilon_{0}^{\prime}-w(2)$ then by the preceding,

$$
w\left(s_{\imath}\right)+w\left(x_{l}\right)+w\left(x_{j}\right) \geq w(v \cdot v)+\varepsilon_{0} ;
$$

for $i=j$, this gives

$$
w\left(x_{i} s_{l} x_{i}^{*}\right) \geq w(v \cdot v)+\varepsilon_{0} .
$$

As this holds for every $i \in I$, it follows that

$$
\operatorname{Min}_{i \in I} w\left(x_{\imath} s_{\iota} x_{\imath}^{*}\right) \geq w(v \cdot v)+\varepsilon_{0}=w\left(\sum_{i \in I} x_{\imath} s_{\imath} x_{\imath}^{*}\right)+\varepsilon_{0} .
$$

Claim 2. Let $\varepsilon_{0}$ be as in Claim 1. Then $V$ is $\varepsilon_{0}$-elliptic.

Let $\left(x_{l}\right),\left(y_{l}\right)$ be any two nullary vectors over $D$. For $i \in I$ fixed:

$$
\begin{aligned}
2 w\left(x_{i} s_{i} y_{l}^{*}\right) & =w\left(x_{i} s_{i} x_{i}^{*}\right)+w\left(y_{l} s_{l} y_{l}^{*}\right) \\
& \geq \operatorname{Min}_{j \in I} w\left(x_{j} s_{j} x_{J}^{*}\right)+\operatorname{Min}_{j \in I} w\left(y_{j} s_{j} y_{J}^{*}\right) .
\end{aligned}
$$

The preceding inequality holding true for every $i \in I$, it follows that

$$
\begin{aligned}
2 \operatorname{Min}_{i \in I} w\left(x_{l} s_{l} y_{l}^{*}\right) & =\operatorname{Min}_{i \in I}\left(2 w\left(x_{l} s_{l} y_{l}^{*}\right)\right) \\
& \geq \operatorname{Min}_{j \in I} w\left(x_{j} s_{J} x_{J}^{*}\right)+\operatorname{Min}_{J \in I} w\left(y_{j} s_{j} y_{J}^{*}\right) \\
& \geq w\left(\sum x_{J} s_{J} x_{J}^{*}\right)+w\left(\sum y_{j} s_{j} y_{J}^{*}\right)+2 \varepsilon_{0} .
\end{aligned}
$$

Given $u, v \in V$ it is clear that there are $\left(x_{l}\right),\left(y_{t}\right)$ such that $\underline{u}=\left(x_{i}\right)$, $\underline{v}=\left(y_{\imath}\right)\left(\right.$ e.g. $\left.u=\sum y_{i} f_{i}\right)$. Then

$$
\begin{aligned}
2 w(u \cdot v) & =2 w\left(\sum x_{i} s_{i} y_{l}^{*}\right) \geq 2 \operatorname{Min}\left(x_{\imath} s_{l} y_{l}^{4}\right) \\
& \geq w\left(\sum x_{j} s_{j} x_{j}^{*}\right)+w\left(\sum y_{j} s_{j} y_{j}^{*}\right)+2 \varepsilon_{0} \\
& =w(u \cdot u)+w(v \cdot v)+2 \varepsilon_{0},
\end{aligned}
$$

and $V$ is $\varepsilon_{0}$-elliptic.

Claim 3. $\left(f_{i}\right)_{t \in I}$ is bounded.

For suppose that $\left(f_{i}\right)$ is $\varepsilon_{0}^{\prime}$-elliptic. Taking up the calculation in Claim 1 , in the case $v=f_{l}+x f_{j}$, where $x$ is to be fixed later and $i, j \in I$ are such that $w(s) \leq w\left(s_{,}\right)$, then

$$
w\left(s_{i}\right)+w(x) \geq w\left(x_{i}+x s_{j} x^{*}\right)+\varepsilon_{0} \quad\left(\varepsilon_{0}=\varepsilon_{0}^{\prime}-w(2)\right) .
$$


Pick $\varepsilon_{1}>\left|\varepsilon_{0}\right|$, where as usual $\left|\varepsilon_{0}\right|=\varepsilon_{0}$ if $\varepsilon_{0} \geq 0$ and $\left|\varepsilon_{0}\right|=-\varepsilon_{0}$ if $\varepsilon_{0}<0$. Put $\varepsilon_{2}=2 \varepsilon_{1}$. I claim that $\infty \neq g \geq \varepsilon_{1}$ implies $w\left(s_{j}\right)-w\left(s_{i}\right) \leq 2 g$ $+\varepsilon_{1}$. For, otherwise, for such a $g \in G$, there is $0 \neq x \in D$ such that $w(x)=-g$. Then

$$
\begin{aligned}
w\left(x s_{j} x^{*}\right) & =w\left(s_{j}\right)-2 g>\left(w\left(s_{i}\right)+2 g+\varepsilon_{1}\right)-2 g \\
& =w\left(s_{i}\right)+\varepsilon_{1}>w\left(s_{\iota}\right) .
\end{aligned}
$$

By the special triangle equality follows

$$
w\left(s_{\imath}+x s_{j} x^{*}\right)=w\left(s_{\iota}\right)
$$

Then

$$
w\left(s_{i}\right)+2(x) \geq w\left(s_{\iota}\right)+\varepsilon_{0}
$$

$-g=w(x) \geq \varepsilon_{0}$. Equivalently, $q \leq-\varepsilon_{0}<\varepsilon_{1}$, contrary to the relation $g \geq \varepsilon_{1}$. Hence the asserted implication holds. If $g=\varepsilon_{1}$, this gives $w\left(s_{j}\right)-$ $w\left(s_{i}\right) \leq s \varepsilon_{1}+\varepsilon_{1}=3 \varepsilon_{1}=\varepsilon_{2}$, as desired. Hence $w\left(s_{j}\right) \geq w\left(s_{l}\right)$ implies $w\left(s_{j}\right)-w\left(s_{i}\right) \leq \varepsilon_{2}$. Now, if $w\left(s_{j}\right)<w\left(s_{i}\right)$ it is clear that since $\varepsilon_{2} \geq 0$, $w\left(s_{j}\right)-w\left(s_{i}\right) \leq \varepsilon_{2}$. Therefore $\left(f_{i}\right)$ is bounded with bound $\varepsilon_{2}$.

(2) $\Rightarrow(1)$. I will establish something more, namely,

$$
\phi w \geq g \Rightarrow \underline{\phi} \underline{w} \geq g+\varepsilon_{0}-\varepsilon^{\prime},
$$

where: $\varepsilon^{\prime}$ is a bound for $\left(f_{i}\right), \varepsilon_{0}$ is an index of ellipticity for $V ; \phi=$ $\operatorname{Mtrx}(\phi)$ - Recall that $\underline{\phi} w \geq g^{\prime}$ means $w\left(\operatorname{entr}_{l, j}(\underline{\phi})\right) \geq g^{\prime}$, for every pair $i, j \in I$.

Claim 1. If $V$ is $\varepsilon_{0}$-elliptic then for each nullary vector $\left(x_{i}\right)$ over $D$,

$$
2 \varepsilon_{0}+w\left(\sum_{\iota \in I} x_{\iota} s_{\iota} x_{\imath}^{*}\right) \geq \operatorname{Min}_{\iota \in I}\left(x_{\imath} s_{\iota} x_{\imath}^{*}\right) .
$$

It suffices to apply inequality (22), Theorem 2.6, $\S 2$, for $v_{l}=x_{i} f_{i}(i \in I)$.

Claim 2. $w \geq g \Rightarrow \underline{\phi} \underline{w} \geq g+\varepsilon_{0}-\varepsilon^{\prime}$.

Put $u_{i}=f_{i} \phi$. From $\phi w \geq g$ follows

$$
w\left(u_{i} \cdot u_{i}\right)=w\left(f_{i} \phi \cdot f_{i} \phi\right) \geq w\left(f_{i} \cdot f_{i}\right)+2 g=w\left(s_{i}\right)+2 g .
$$

Now,

$$
w\left(u_{\imath} \cdot u_{\iota}\right)=w\left(\sum_{j \in I} \phi_{\iota s} s_{j} \phi_{\iota j}^{\prime}\right)
$$


From Claim 1, follows

$$
\begin{gathered}
w\left(\phi_{i j^{\prime}} s_{j^{\prime}} \phi_{i j^{\prime}}^{*}\right) \geq \operatorname{Min}_{j \in I} w\left(\phi_{i j} s_{j} \phi_{i j}^{*}\right) \geq w\left(\sum \phi_{i j} s_{j} \phi_{i j}^{*}\right)+2 \varepsilon_{0} \\
=w\left(u_{i} \cdot u_{i}\right)+2 \varepsilon_{0} \geq w\left(s_{i}\right)+2 g+2 \varepsilon_{0} \\
2 w\left(\phi_{i j^{\prime}}\right) \geq 2\left(s_{i}\right)-w\left(s_{j}\right)+2 g+2 \varepsilon_{0} \\
\geq-\varepsilon^{\prime}+2 g+2 \varepsilon_{0} \geq-2 \varepsilon^{\prime}+2 g+2 \varepsilon_{0} \\
w\left(\phi_{i j^{\prime}}\right) \geq g+\varepsilon_{0}-\varepsilon^{\prime}
\end{gathered}
$$

as desired.

Claim 3. If $\phi \in U$ then $\underline{\phi} \underline{w} \geq 2 \varepsilon_{0}-\varepsilon^{\prime}$.

Since $V$ is $\varepsilon_{0}$-elliptic, it follows that

$$
2 w(u \cdot v \phi) \geq w(u \cdot u)+w(v \phi \cdot v \phi)+2 \varepsilon_{0},
$$

and since $\phi$ is unitary,

$$
\begin{aligned}
2 w(u \cdot v \phi) & \geq w(u \cdot u)+2(v \phi \cdot v \phi)+2 \varepsilon_{0} \\
& =w(u \cdot u)+w(v \cdot v)+2 \varepsilon_{0}
\end{aligned}
$$

so that,

$$
\phi w \geq \varepsilon_{0} .
$$

By Claim 2,

$$
\underline{\phi} \underline{w} \geq \varepsilon_{0}+\left(\varepsilon_{0}-\varepsilon^{\prime}\right)=2 \varepsilon_{0}-\varepsilon^{\prime}
$$

follows.

The proof of the preceding theorem revealed few facts that are appropriate to separate for future reference. This is the content of

REMARKS 3.3. Let $\left(f_{i}\right)$ be any orthogonal basis of the non-isotropic unitary space $V$. Then:

(a) If $V$ is $\varepsilon_{0}$-elliptic and if $\varepsilon_{1}$ is a bound for $\left(f_{i}\right)$, then

$$
\phi w \geq g \Rightarrow \underline{\phi} \underline{w} \geq\left(g+\varepsilon_{0}-\varepsilon_{1}\right) .
$$

(b) If $V$ is $\varepsilon_{0}$-elliptic then for every nullary vector $\left(x_{i}\right)$ over $D$,

$$
w\left(\sum_{i \in I} x_{i} s_{i} x_{i}^{*}\right)-2 \varepsilon_{0} \leq \operatorname{Min}_{i \in I}\left(w\left(x_{l} s_{l} x_{i}^{*}\right)\right) .
$$


(c) Conversely, if the preceding inequality holds then $V$ automatically verifies a generalized Cauchy-Schwarz inequality. In effect, $V$ is $\left(\varepsilon_{0}\right)$ elliptic.

I proceed to a converse of inequality (12) in Remarks 3.3.

THEOREM 3.4. Let $V$ be any non-degenerate $\varepsilon_{0}$-elliptic space with orthogonal basis $\left(f_{\iota}\right)$ bounded by $\varepsilon_{1}$. If $\phi \in L$ is such that $\underline{\phi} \underline{w} \geq g$, then $\phi w \geq g+2 \varepsilon_{0}-\varepsilon_{1}$ follows.

Proof. As a first reduction, one can replace the asserted relation by the quadratic inequality

$$
w(v \phi \cdot v \phi) \geq w(v \cdot v)+2\left(g+\varepsilon_{0}-\varepsilon_{1}\right) .
$$

This readily follows from $\varepsilon_{0}$-ellipticity of $V$. As a second reduction, one may assume $V$ is finite-dimensional. The argument to that effect runs as follows. If $\underline{v}$ is the row vector of $v \in V$ and $\underline{v \phi}$ that of $v \phi$, then for $\underline{u}=\underline{v \phi}$ it is clear that

$$
\underline{v} \underline{u}=\underline{u} .
$$

By construction, $\underline{u}$ is a nullary vector over $D$. If $\underline{F}^{(\iota, j)}$ is the usual $(i, j)$ standard matrix in $D_{I \times I}$ and $\phi_{i j}=\operatorname{entr}_{\iota, j}(\phi)$ then evidently there is a finite subset $I_{1}$ of $I$ such that if $\Phi^{\prime}=\sum_{i, j \in I_{1}} \bar{E}^{(i, j)} \phi_{\iota j}$ then

$$
\underline{u}=\underline{v} \phi^{\prime} .
$$

If $\phi^{\prime} \in L$ is such that $\Phi^{\prime}=\operatorname{Mtrx}\left(\phi^{\prime}\right)$, then $v \phi^{\prime}=v \phi$ follows. By construction, $\Phi^{\prime}=\phi^{\prime} \underline{w} \geq g$. If the assertion can be shown for $\phi$ replaced by $\phi^{\prime}$ then evidently

$$
w(v \phi \cdot v \phi)=w\left(v \psi^{\prime} \cdot v \phi^{\prime}\right) \geq w(v \cdot v)+2\left(g+\varepsilon_{0}-\varepsilon_{1}\right) .
$$

Hence one can replace $\phi$ by $\phi^{\prime}$. If $V^{(1)}$ is the subspace that is spanned by the $f_{i}$ as $i$ ranges over $I_{1}$, then without loss of generality $v \in V^{(1)}$, by construction $V^{(1)} \phi^{\prime} \subset V^{(1)}$, and $\left(f_{\imath}\right)_{\iota \in I_{1}}$ is, again, orthogonal with bound $\varepsilon_{1}$. Since $V^{(1)}$ is finite-dimensional the asserted reduction follows. Hence in what follows $I$ can be taken to be a finite set, say, $I=\{1,2, \ldots, n\}$. Let $S=\operatorname{Diag}\left\{s_{1}, \ldots, s_{n}\right\}$. Then

$$
v \phi \cdot v \phi=\underline{v \phi S}\left(\underline{v \phi}^{\mathrm{tr} . *}\right)
$$

so that

$$
w(v \phi \cdot v \phi)=w\left(\underline{v \phi} S(\underline{v \phi})^{\operatorname{tr} . *}\right) .
$$


Observing that if $\underline{w}(\Phi)$ is the minimum value of the entries of $\Phi$ then $\underline{w}$ is sub-multiplicative one gets

$$
w\left(\underline{v \phi} S(\underline{v \phi})^{\operatorname{tr} . *}\right)=\underline{w}\left(\underline{v \phi} S(\underline{v \phi})^{\operatorname{tr} . *}\right) \geq 2 \underline{w}(\underline{v \phi})+\underline{w}(S) .
$$

Hence,

$$
w(v \phi \cdot v \phi) \geq w \underline{w}(\underline{v \phi})+w(S) \geq 2 \underline{w}(\underline{v})+2 \underline{w}(\underline{\phi})+\underline{w}(S) .
$$

If $v=\sum x_{\imath} f_{\imath}$, then by Remarks 3.3b) follows

$$
\operatorname{Min} w\left(x_{\iota} s_{\iota} x_{\iota}^{*}\right) \geq w\left(\sum x_{\iota} s_{\iota} x_{\imath}^{*}\right)+2 \varepsilon_{0}=w(v \cdot v)+2 \varepsilon_{0} .
$$

If $\bar{w}(S)$ is the maximum value of the diagonal entries in $S($ all $\neq 0$ ), then

$$
2 \underline{w}(\underline{v})+\bar{w}(S) \geq \operatorname{Min} w\left(x_{\imath} s_{\imath} x_{\imath}^{*}\right) \geq w(v \cdot v)+2 \varepsilon_{0} .
$$

Hence,

$$
\begin{aligned}
w(v \phi \cdot v \phi) & \geq\left(w(v \cdot v)+2 \varepsilon_{0}-\bar{w}(S)\right)+2 \underline{w}(\underline{\phi})+\underline{w}(S) \\
& \geq w(v \cdot v)+2 \varepsilon_{0}-\overline{2}(S)+\underline{w}(S)+2 g \\
& =w(v \cdot v)+2 \varepsilon_{0}+(\underline{w}(S)-\bar{w}(S))+2 g \\
& \geq w(v \cdot v)+2 \varepsilon_{0}-\varepsilon_{1}+2 g \\
& \geq w(v \cdot v)+2 \varepsilon_{0}-2 \varepsilon_{1}+2 g \\
& =w(v \cdot v)+2\left(g+\varepsilon_{0}-\varepsilon_{1}\right),
\end{aligned}
$$

as desired.

Combining (12) with Theorem 3.4:

THEOREM 3.5. Let $V$ be any non-degenerate $\varepsilon_{0}$-elliptic space and let $\left(f_{i}\right)$ be any orthogonal basis, which is bounded by $\varepsilon_{1}$. Then:

1. $\phi w \geq \Rightarrow \underline{w} \underline{w} \geq \underline{g}\left(=g+2 \varepsilon_{0}-\varepsilon_{1}\right)$;

2. $\underline{\phi} \underline{w} \geq g \stackrel{\Rightarrow}{\Rightarrow} \underline{ } \geq \underline{g}$; where $\phi$ is the matrix of $\phi$ and $\underline{\phi} \underline{w} \geq g$ means $w\left(\operatorname{entr}_{i, j}(\underline{\phi})\right) \geq g$ for every pair $1, j \in I$.

COROLlaRy 3.6. Under the assumptions in Theorem 3.5, if $g_{1}, g_{2} \in G$ are such that $\left|g_{1}-g_{2}\right|>2\left(\varepsilon_{1}-2 \varepsilon_{0}\right)$ then $L_{g_{1}} \neq L_{g_{2}}$.

Proof. Assume, say, $g_{2}>g_{1}+2\left(\varepsilon_{1}-2 \varepsilon_{0}\right)$. If now $L_{g_{1}}=L_{g_{2}}$ define $\bar{g}_{1}=g_{1}-2 \varepsilon_{0}+\varepsilon_{1}$. If $\left(J_{\bar{g}_{1}}\right)_{I \times I}$ is the additive subgroup of $I \times I$ matrices $\Phi \in D_{I \times I}$ such that each entry of $\Phi$ belongs to $J_{\bar{g}}$ then by Theorem 3.5, point 2. follows $\operatorname{Mtrx}^{-1}\left(J_{\bar{g}_{1}}\right)_{I \times I} \subset L_{g_{1}}$. Also, by Theorem 3.5, point 1 . 
follows $\operatorname{Mtrx}^{-1}\left(J_{\underline{g}_{2}}\right) \supset L_{g_{2}}$. Thus

$$
\operatorname{Mtrx}^{-1}\left(J_{\underline{g}_{2}}\right)_{I \times I} \operatorname{Mtrx}^{-1}\left(J_{\bar{g}_{1}}\right)_{I \times I} .
$$

Hence,

$$
\left(J_{\underline{g}_{2}}\right)_{I \times I} \supset\left(J_{\bar{g}_{1}}\right)_{I \times I} \text {. }
$$

Thus,

$$
J_{g_{2}} \supset J_{\bar{g}_{1}} \cdot
$$

From this $\underline{g}_{2} \leq \bar{g}_{1}$. Recalling that $\underline{g}_{2}=g_{2}+2 \varepsilon_{0}-\varepsilon_{1}$ and $\bar{g}_{1}=g_{1}-2 \varepsilon_{0}$ $+\varepsilon_{1}$, then

$$
g_{2}-g_{1} \leq\left(-2 \varepsilon_{0}+\varepsilon_{1}\right)
$$

which is a contradiction.

In the course of the proof of the preceding corollary, certain matrix approximation of the additive subgroup $L_{g}$ was offered; namely,

$$
\operatorname{Mtrx}\left(L_{g}\right) \supset\left(J_{\bar{g}}\right)_{I \times I} \quad\left(\bar{g}=g-2 \varepsilon_{0}+\varepsilon_{1}\right) .
$$

One can offer related bound for the congruence subgroup $U_{g}$ of the unitary group $U$. For observe that the passage from $\phi \in(L ; *)$ to $\operatorname{Mtrx}(\phi)$ $\in\left(D_{I \times I} ;(S)\right)$ is an isomorphism of partial involutorial rings. Hence

$$
\operatorname{Mtrx}(U)=\mathscr{U}\left(D_{I \times I} ;(S)\right) \quad\left(=\text { Unitary group of }\left(D_{I \times I} ;(S)\right)\right) \text {. }
$$

Related to the congruence subgroup $U_{g}$ (resp. $U_{g}^{+}$) is the additive subgroup $\mathscr{K}_{g}(V)$ (resp. $\mathscr{K}_{g}^{+}(V)$ ) which, I recall, is the set of skew-symmetric transformations $\psi=-\psi^{*}$ such that $\psi \in L_{g}(V)$ (resp. $\psi \in L_{g}^{+}(V)$ ).

REMARK 3.7. In the partial involutorial matrix ring $D_{I \times I}$ relative to $(S)$, the matrix $\Phi$ is skew-symmetric if, and only if, $\Phi$ is of the form

$$
\Phi=S \Sigma,
$$

where $\Sigma$ is a row and column finite matrix such that

$$
\Sigma^{\text {tr.** }}=-\Sigma \text {. }
$$

Proof. From (18) follows

$$
\Phi^{(S)}=(S \Sigma)^{(S)}=S\left(\Sigma^{\mathrm{tr} . *} S\right) S^{-1}=-S \Sigma .
$$

Conversely, if $\Phi^{(S)}$ is defined then $\Phi$ is both row and column finite and then

$$
\Phi^{(S)}=S \Phi^{\text {tr.**}} S^{-1}=-\Phi .
$$


If $\Sigma=S^{-1} \Phi$, then by construction $\Phi=S \Sigma$ and,

$$
\Sigma^{\text {tr.**}}=\Phi^{\text {tr.** }} S^{-1}=-S^{-1} \Phi \quad(\text { from }(19))=-\Sigma .
$$

THEOREM 3.9. Let $V$ be as in Theorem 3.5. Assume, further, that $V$ is not the 1-dimensional orthogonal space. If $g_{1}, g_{2} \in G$ are such that $\left|g_{1}-g_{2}\right|$ $>3 \varepsilon_{1}-4 \varepsilon_{0}$ and if $g_{1}, g_{2}>w(2)$ then $U_{g_{1}} \neq U_{g_{2}}$.

Proof. Let $\mathscr{K}_{g}\left(D_{I \times I} ;(S)\right)$ be the additive subgroup of skew-symmetric matrices $\Phi$ relative to $(S)$ such that $\Phi \underline{w} \geq g$. From Theorem 3.5, point 2., follows

$$
\operatorname{Mtrx}\left(\mathscr{K}_{g}(V)\right) \supset_{\bar{g}}\left(D_{I \times I} ;(S)\right),
$$

where $\bar{g}=g-2 \varepsilon_{0}+\varepsilon_{1}$. From the cited theorem point 1 . follows

$$
\operatorname{Mtrx}\left(\mathscr{K}_{g}(V)\right) \subset \mathscr{K}_{\underline{g}}\left(D_{I \times I} ;(S)\right),
$$

where $\underline{g}=g+2 \varepsilon_{0}-\varepsilon_{1}$. Assume that $\mathscr{K}_{g_{1}}(V)=\mathscr{K}_{g_{2}}(V)$. Applying (20) to $g=g_{1}$ and (21) to $g=g_{2}$ this gives

$$
\mathscr{K}_{\bar{g}_{1}}\left(D_{I \times I} ;(S)\right) \subset \operatorname{Mtrx}\left(\mathscr{K}_{g_{1}}(V)\right)=\operatorname{Mtrx}\left(\mathscr{K}_{g_{2}}(V)\right) \subset \mathscr{K}_{g_{2}}\left(D_{I \times I} ;(S)\right) .
$$

From Remark 3.7 follows

$$
\mathscr{K}_{g}\left(D_{I \times I} ;(S)\right)=\left\{S \Sigma \mid \Sigma^{\mathrm{tr} . *}=-\Sigma(S \Sigma) \underline{w} \geq g\right\} .
$$

When the ground involution $*$ in $D$ is the identity mapping then by hypothesis $\operatorname{dim}_{D} V \geq 2$. In that case choose $\Sigma$ of the form

$$
\Sigma=\left|\begin{array}{rr}
0 & x \\
-x & 0
\end{array}\right| \oplus 0
$$

where $w\left(s_{1} x\right)$ and $w\left(s_{2} x\right) \geq \bar{g}_{1}$. By construction, $S \Sigma \in \mathscr{K}_{\bar{g}_{1}}\left(D_{I \times I} ;(S)\right)$. Hence, $S \Sigma \in \mathscr{K}_{g_{2}}\left(D_{I \times I} ;(S)\right)$ and so, $w\left(s_{1} x\right)$ and $w\left(s_{2} x\right) \geq \underline{g}_{2}$. What I have shown is that

$$
\left\{\begin{array} { l } 
{ w ( s _ { 1 } x ) \geq \overline { g } _ { 1 } } \\
{ w ( s _ { 2 } w ) \geq \overline { g } _ { 1 } }
\end{array} \quad \Rightarrow \left\{\begin{array}{l}
w\left(s_{1} x\right) \geq \underline{g}_{2} \\
w\left(s_{2} x\right) \geq \underline{g}_{2}
\end{array} \quad(x \in D) .\right.\right.
$$

Now $\left|w\left(s_{1}\right)-w\left(s_{2}\right)\right| \leq \varepsilon_{1}$. If then $x$ is such that $w(x)=\bar{g}_{1}-w\left(s_{1}\right)+\varepsilon_{1}$, then

$$
w\left(s_{1} x\right)=\bar{g}_{1}+\varepsilon_{1}>\bar{g}_{1}
$$

and

$$
\begin{aligned}
w\left(s_{2} x\right) & =w(x)+w\left(s_{2}\right)=\bar{g}_{1}+\varepsilon_{1}+\left(w\left(s_{2}\right)-w\left(s_{1}\right)\right) \\
& \geq \bar{g}_{1}+\varepsilon_{1}-\varepsilon_{1}=\bar{g}_{1}
\end{aligned}
$$


From (22) follows $w\left(s_{1} x\right)=\bar{g}_{1}+\varepsilon_{1} \geq \underline{g}_{2}$. Hence,

$$
\bar{g}_{1}+\varepsilon_{1}=g_{1}-2 \varepsilon_{0}+2 \varepsilon_{1} \geq \underline{g}_{2}+2 \varepsilon_{0}-\varepsilon_{1} \text {. }
$$

or,

$$
g_{1}-g_{2} \geq 4 \varepsilon_{0}-3 \varepsilon_{1} ; g_{2}-g_{1} \geq 3 \varepsilon_{1}-4 \varepsilon_{0} .
$$

By symmetry,

$$
g_{1}-g_{2} \leq 3 \varepsilon_{1}-4 \varepsilon_{0}
$$

contradicting the hypothesis $\left|g_{1}-g_{2}\right|>3 \varepsilon_{1}-4 \varepsilon_{0}$.

When the ground involution $*$ in $D$ is not the identity mapping one can choose in that case $\Sigma$ to be of the form

$$
\Sigma=[x] \oplus 0,
$$

where $x^{*}=-x$. By a trivial adaptation of the preceding argument follows $\left|g_{1}-g_{2}\right| \leq 2 \varepsilon_{1}-4 \varepsilon_{0}$, which contradicts again the hypothesis. This shows that $\mathscr{K}_{g_{1}}(V) \neq \mathscr{K}_{g_{2}}(V)$. Since $g_{1}, \iota_{2}>w(2)$ it follows from Theorem 2.16, §2, that $U_{g_{1}} \neq U_{g_{2}}$.

The proof of the preceding theorem evidenced the fact that the smaller the bounds $\varepsilon_{0}, \varepsilon$ the better the matrix counterparts of $L_{g}, U_{g}$ will be. Hence, it seems desirable to work out still further the special case where the basis $\left(f_{l}\right)$ is 0 -elliptic which means, I recall, every unitary transformation $\phi$ of $V$ has matrix over the valuation ring $R$. From Theorem 3.2, the space $V$ automatically verifies a generalized CauchySchwarz inequality where the index is not less than $-w(2)$. When $1 / 2 \in R$, then $V$ becomes 0 -elliptic and the bound $\varepsilon$ for $\left(f_{\iota}\right)$ should be quite small since by the cited theorem $\left|w\left(s_{\imath}\right)-\left(s_{j}\right)\right| \leq 2 g$ for each positive $g \in G$. In the theorem to follow it is shown that indeed $\varepsilon$ can be taken to be either 0 or else, $\varepsilon$ is the first positive element in $G$. This is the

THEOREM 3.10. Let $V$ be any non-isotropic unitary space with orthogonal $\operatorname{basis}\left(f_{\imath}\right)$. If $1 / 2 \in R$ and if the basis is 0 -elliptic then:

(1) $V$ is 0 -elliptic.

(2) The basis $\left(f_{i}\right)$ is bounded by $\varepsilon$, where either $\varepsilon=0$ or else $\varepsilon$ is the first positive element in the value group $G$.

Proof (of (2)). If the basis $\left(f_{i}\right)$ is uniform in the technical sense all the $f_{i}$ have same lengths there is nothing more to prove. If to the contrary there is a pair $i, j \in I$ such that $f_{i}$ and $f_{j}$ have distinct lengths then $\left|w\left(s_{t}\right)-w\left(s_{j}\right)\right| \neq 0$. Since $\left|w\left(s_{i}\right)-w\left(s_{j}\right)\right| \leq 2 g$ for every $g>0$ it follows 
that if $\varepsilon_{1}=\left|w\left(s_{i}\right)-w\left(s_{j}\right)\right|$ then $\varepsilon_{1}$ is the first positive element of $G$ if $\varepsilon_{1} \notin 2 G$; otherwise, $1 / 2 \varepsilon_{1}$ is the first positive element of $G$. In both cases, $G$ has definitely a first positive element $\varepsilon$ and what is left to show is that the case $\varepsilon_{1} \in 2 G$ can be dismissed. For assume, say, $w\left(s_{j}\right)=w\left(s_{i}\right)+2 \varepsilon$. Evidently $V$ has dimension at least 2 . Pass to the 2-dimensional subspace $V^{(1)}$ spanned by $f_{j}$ and $f_{i}$. Then $V^{(1)}$ has orthogonal complement in $V$. by standard argument, $\left(f_{i}, f_{j}\right)$ is then a 0 -elliptic basis for the space $V^{(1)}$. Put $v=x_{1} f_{i}+x_{2} f_{j}$, where $x_{1}, x_{2} \in D$ are to be fixed later. Writing out the matrix of the projection $\tau$ induced by $v$ one gets for matrix of $\tau$ the following matrix:

$$
\operatorname{Mtrx}(\tau)=\left|\begin{array}{ll}
s_{i} x_{1}^{*} \frac{1}{v \cdot v} x_{1} & s_{i} x_{1}^{*} \frac{1}{v \cdot v} x_{2} \\
s_{j} x_{2}^{*} \frac{1}{v \cdot v} x_{1} & s_{j} x_{2}^{*} \frac{1}{v \cdot v} x_{2}
\end{array}\right|
$$

Since by hypothesis $w(2)=0$ it follows as observed earlier that $\operatorname{Mtrx}(\tau) \underline{w} \geq 0$ and, hence, $w\left(\operatorname{entr}_{1,2}(\operatorname{Mtrx}(\tau))\right) \geq 0$. Since $V$ is a 0 -elliptic space one knows in that case that (Theorem 1.7, $\$ 1$, equality $(29)^{\prime}$ )

$$
w(v \cdot v)=w\left(x_{1} s_{i} x_{1}^{*}+x_{2} s_{j} x_{2}^{*}\right)=\operatorname{Min}\left(w\left(x_{1} s_{i} x_{1}^{*}\right), w\left(x_{2} s_{j} x_{2}^{*}\right)\right) .
$$

If $x_{1}, x_{2}$ are such that $w\left(x_{1}\right)=0, w\left(x_{2}\right)=-\varepsilon$ then one should get for such $x_{i}, \operatorname{entr}_{1,2}\left(\underline{\tau}^{(1)}\right) \in R$. Equivalently,

$$
w\left(s_{i}\right)+w\left(x_{1}\right)+w\left(x_{2}\right) \geq w(v \cdot v) .
$$

Now,

$$
\begin{aligned}
w\left(x_{2} s_{j} x_{2}^{*}\right) & =2 w\left(x_{2}\right)+2\left(s_{j}\right)=-2 \varepsilon+w\left(s_{j}\right) \\
& =(-2 \varepsilon)+\left(2\left(s_{i}\right)+2 \varepsilon\right)=w\left(s_{i}\right) \\
& =w\left(s_{i}\right)+2 w\left(x_{1}\right)=w\left(x_{1} s_{i} x_{1}^{*}\right) .
\end{aligned}
$$

Hence,

$$
\operatorname{Min}\left(w\left(x_{1} s_{i} x_{1}^{*}\right), w\left(x_{2} s_{j} x_{2}^{*}\right)\right)=w\left(s_{i}\right)
$$

Thus,

$$
w(v \cdot v)=w\left(s_{i}\right) .
$$

Substituting for $w(v \cdot v)$ in the preceding inequality this gives

$$
w\left(x_{1}\right)+w\left(x_{2}\right) \geq 0 \text { or } 0-\varepsilon \geq 0,
$$

which is nonsense.

THEOREM 3.11. Conversely, let $V$ be any non-degenerate 0-elliptic space $-w(2)$ need not be 0 . If $\left(f_{i}\right)_{i \in I}$ is any orthogonal basis with bound $\varepsilon$, where $\varepsilon=0$ or $\varepsilon$ is the first positive element in $G$, then $\left(f_{i}\right)_{i \in I}$ is a 0 -elliptic basis for $V$. 
Proof. It is to be shown that if $\phi \in U$ then $\operatorname{Mtrx}(\phi) \in R_{I \times I}$. I will show something more, namely, if $\phi$ is integral (e.g. $\left.\phi \in L_{0}\right)$ then $\operatorname{Mtrx}(\phi)$ $\in R_{I \times I}$. For let $\phi \in L_{0}$. Then $\phi$ increases lengths. Hence, for each $i \in I$,

$$
w\left(f_{i} \phi \cdot f_{i} \phi\right) \geq w\left(s_{i}\right) .
$$

If $\phi_{i j}$ is the $(i, j)$ entry in the matrix $\underline{\phi}$ of $\phi$ then as in the proof of Theorem 3.2, point 1, Claim 2, one has

$$
\begin{aligned}
w\left(\phi_{i j} s_{j} \phi_{i j}^{*}\right) & \geq \operatorname{Min}_{j_{0} \in I} w\left(\phi_{i j_{0}} s_{i j_{0}} \phi_{i j_{0}}^{*}\right) \\
& =w\left(\sum_{j_{0} \in I} \phi_{i j_{0}} s_{j 0} \phi_{i j_{0}}^{*}\right)=w\left(f_{i} \phi \cdot f_{i} \phi\right) \geq w\left(s_{i}\right) .
\end{aligned}
$$

Hence,

$$
2 w\left(\phi_{i j}\right) \geq w\left(s_{i}\right)-w\left(s_{j}\right) \geq-\varepsilon .
$$

If $\varepsilon=0$ from $2 w\left(\phi_{i j}\right) \geq 0$ follows $w\left(\phi_{i j}\right) \geq 0$. If $\varepsilon$ is the first positive element of $G$, then by the usual arithmetic argument from $2 w\left(\phi_{i j}\right) \geq-\varepsilon$ follows $w\left(\phi_{i j}\right) \geq 0$, as desired.

If now $\phi$ is any unitary matrix then from 0 -ellipticity of $V$ follows $\phi \in L_{0}$ so that $\operatorname{Mtrx}(\phi) \in R_{I \times I}$.

To recapitulate the foregoing theorems in the special case under consideration:

THEOREM 3.12. Let $V$ be any non-isotropic unitary space. Suppose that $1 / 2 \in R$ and let $\left(f_{\iota}\right)$ be any orthogonal basis of $V$. The following requirements are equivalent.

(1) $V$ is 0 -elliptic and $\left(f_{\iota}\right)$ has bound $\varepsilon$ with $\varepsilon$ either 0 or else $\varepsilon$ is the first positive element in $G$.

(2) If $\phi$ is unitary transformation then the matrix of $\phi$ is over the valuation ring $R$ in $D$.

(3) If $\phi$ increases lengths $(e . g \cdot w(v \phi \cdot v \phi) \geq w(v \cdot v)$ for every $v \in V)$ then $\operatorname{Mtrx}(\phi)$ is over $R$.

(4) $(\phi w \geq \imath \Rightarrow \operatorname{Mtrx}(\phi) \underline{w} \geq g)$ and $(\operatorname{mtrx}(\phi) \underline{w} \geq g \Rightarrow \phi w \geq g-\varepsilon)$.

Although Theorem 3.12 point 4 . asserts that the prevaluations $\cdots w$ $\geq \cdots$ and $\cdots \underline{w} \geq \cdots$ can be identified up to $\varepsilon$, still, in the inequality

$$
\operatorname{Mtrx}(\phi) \underline{w} \geq g \Rightarrow \phi w \geq g-\varepsilon,
$$

it would be wrong to drop the term $-\varepsilon$. For if one neglects it, then $w$ and $\underline{w}$ would be isomorphic $*$-prevaluations. Hence $\underline{w}$ would be an $(S)$-prevaluation; by inspection, this happens exactly when $S$ has all its diagonal 
entries with same values. Equivalently, the basis $(f)$ is uniform (i.e. all $f_{i}$ have same lengths). Because of the residual term $-\varepsilon$ one has less control on the strict prevaluation $\cdots w>\cdots$ and it is again wrong to infer that $\phi w>g$ implies $\phi \underline{w}>g$. The kind of information which is lacking here is how one can decide whether or not $\phi \in L_{0}$ looking at the matrix of $\phi$. This problem will be solved in what will follow; but, first some additional notations and definitions.

(g) Uniform and mixed bases.

Without loss of generality the index set $I$ can be turned into a linearly ordered set such that

$$
i \geq j \Rightarrow w\left(s_{i}\right) \geq w\left(s_{j}\right) .
$$

By construction, if $E=\left\{w\left(s_{\iota}\right)\right\}_{\iota \in I}$, then either

$$
\# E=1 \text { (uniform basis), }
$$

or

$$
\# E=2 \quad \text { (mixed basis) }
$$

In the case the basis is uniform, put

$$
I_{0}=I, \quad I_{\varepsilon}=\varnothing .
$$

In the case the basis is mixed, put

$$
I_{0}=\left\{i \in I \mid w\left(s_{i}\right)=\operatorname{Min}(E)\right\}, \quad I_{\varepsilon}=\left\{i \in I \mid w\left(s_{i}\right)=\operatorname{Min}(E)\right\} .
$$

By construction, $I$ is the ordinal product of the ordered subsets $I_{0}$ and $I_{\varepsilon}$. As a memonic device for the considered matrix constructions to follow let me make the

(h) Convention. Given $g=-\varepsilon$, 0 , or $\varepsilon$ let

$$
J^{g}= \begin{cases}R, & \text { if } g=-\varepsilon \text { or } 0, \\ J, & \text { if } g=\varepsilon\end{cases}
$$

For instance, if $G$ is the ordered additive group of integers then:

$$
J^{0}=R ; \quad J^{1}=J
$$

and by convention,

$$
J^{-1}=R \text {. }
$$

There shall not be confusion in what will follow with inverses of fractional ideals. 
(i) $w$-Matrix. By $w$-matrix, I mean any $I \times I$ matrix $W$ over the value group $G$ such that if $w_{i j}=\operatorname{entr}_{i, j}(W)$, then:

(i) If $G$ has no first positive element then $w_{i j}=0$ for every pair $i, j \in I .(0=$ zero of $G)$;

(ii) If $G$ has a first positive element $\varepsilon$, then $w_{i j}=-\varepsilon, 0$ or $\varepsilon$, and;

(iii) $i \geq j \Rightarrow w_{i j} \geq 0$;

(iv) $w_{j i}=-w_{i j}(i, j \in I)$.

(j) The ring $R_{W}$. Given the $w$-matrix $W$ over $G$ denote by $R_{W}$ the subset of matrices $\Phi=\left[\phi_{i j}\right]_{i, j \in I}$ such that

$$
\left\{\begin{array}{l}
\phi_{i j} \in R^{i j} \quad(\text { all } i, j \in I), \\
\Phi \in D_{I \times I}
\end{array}\right.
$$

By inspection, $R_{W}$ is a subring of $D_{I \times I}$, which is closed under the partial matrix involution $(S)$.

(k) The ideal $J_{W}$. Given the $w$-matrix $W$, denote by $W^{+}$the $I \times I$ matrix over $G$ such that if $w_{i j}^{+}=\operatorname{entr}_{i, j}(W)$ then

$$
w_{i j}^{+}= \begin{cases}w_{i j}, & \text { if } w_{i j} \neq 0, \\ w_{i j}^{+}, & \text {if } w_{i j}=0 .\end{cases}
$$

Define $J_{W}$ to be the subset of matrices $\Phi=\left[\phi_{i j}\right]_{i, j \in I}$ such that

$$
\left\{\begin{array}{l}
\phi_{i j} \in R^{w_{i j}^{+}} \\
\Phi \in D_{I \times I}
\end{array} \quad(\text { all } i, j \in I),\right.
$$

By inspection, $J_{W}$ is a $(S)$-closed 2-sided ideal of the ring $R_{W}$.

(1) Illustration. Every orthogonal basis $\left(f_{i}\right)$ of the non-isotropic space $V$ such that $\left(f_{i}\right)$ is bounded by the considered $\varepsilon$ gives rise to the $w$-matrix $w=w\left(\left(f_{i}\right)\right)$, where

$$
w_{i j}=w\left(s_{i}\right)-w\left(s_{j}\right) \quad\left(s_{k}=f_{k} \cdot f_{k}, k \in I\right) .
$$

Hence, if $I$ is the ordered segment $I=\{1,2,3,4\}, I_{0}=\{1,2\}$ and $I_{\varepsilon}=$ $\{3,4\}$, then:

$$
\begin{aligned}
W & =\left|\begin{array}{cc:cc}
0 & 0 & -\varepsilon & -\varepsilon \\
0 & 0 & -\varepsilon & -\varepsilon \\
\hdashline \varepsilon & \varepsilon & 0 & 0 \\
\varepsilon & \varepsilon & 0 & 0
\end{array}\right| ; & W^{+}=\left|\begin{array}{cc:cc:c}
\varepsilon & \varepsilon & -\varepsilon & -\varepsilon \\
\varepsilon & \varepsilon & -\varepsilon & -\varepsilon \\
\hdashline \varepsilon & \varepsilon & \varepsilon & \varepsilon \\
\varepsilon & \varepsilon & \varepsilon & \varepsilon
\end{array}\right| ; \\
R_{W} & =\left|\begin{array}{lllll}
R & R & R & R \\
R & R & R & R \\
\hdashline J & J & R & R \\
J & J & R & R
\end{array}\right| ; & J_{W}=\left|\begin{array}{lllll}
J & J & R & R \\
J & J & R & R \\
\hdashline J & J & J & J \\
J & J & J & J
\end{array}\right| .
\end{aligned}
$$


That, again, $R_{W}$ is an $(S)$-closed subring of $D_{I \times I}$ and $J_{W}$ is an $(S)$-closed 2-sided ideal of $R_{W}$ this is also a consequence of the

THEOREM 3.13. Let $V$ be any non-degenerate 0-elliptic space with orthogonal basis $\left(f_{i}\right)_{i \in I}$ and suppose that either $\left(f_{i}\right)$ is uniform or $\left(f_{i}\right)$ is mixed (e.g. $\left(f_{i}\right)$ is bounded by $\varepsilon$ where either $\varepsilon=0$ or $\varepsilon=1$ st positive element of the value group $G)$. If $W$ is the corresponding $w$-matrix $\left(w_{i j}=\right.$ $\left.\operatorname{entr}_{\iota, j}(W)=w\left(s_{i}\right)-w\left(s_{j}\right), s_{k}=f_{k} \cdot f_{k}\right)$ then:

(1) The ring $R_{W}$ of all row-finite $I \times I$ matrices $\Phi=\left[\phi_{i j}\right]$ with $\phi_{i j} \in J^{w_{i j}}$ is isomorphic to the ring $L_{0}$ of linear transformations $\phi$ increasing lengths.

(2) The ideal $J_{W}$ of all row-finite $I \times I$ matrices $\Phi=\left[\phi_{i j}\right]$ with $\phi_{i j} \in J^{w_{i j}^{+}}$ is isomorphic to the ideal $L_{0}^{+}$of linear transformations $\phi$ strictly increasing lengths.

\section{Proof. Claim 1. $\operatorname{Mtrx}\left(L_{0}\right) \subset R_{W}$.}

From Theorem 3.2 implication (2) $\Rightarrow(1)$, claim 2, follows that if $\phi \in L_{0}$ then if $\operatorname{Mtrx}(\phi)=\left[\phi_{i j}\right]$ then $2 w\left(\phi_{i j}\right) \geq w\left(s_{i}\right)-w\left(s_{j}\right)=w_{i j}$.

If $w_{i j} \leq 0$ then $J^{w_{i j}}=R$ and, hence, $\phi_{i j} \in J^{w_{i j}}$. If $w_{i j}>0$ then $w_{i j}=\varepsilon$ so that $2 w\left(\phi_{i j}\right) \geq \varepsilon$. From this $w\left(\phi_{i j}\right) \geq \varepsilon$ and, consequently, $\phi_{i j} \in J=$ $R^{w_{t}}$.

Claim 2. Turn $V$ into a right vector space over the division ring $D$ using the basis $\left(f_{i}\right)$ as follows:

$$
v=\sum_{i \in I} x_{i} f_{i}, \quad \lambda \in D \rightarrow v \lambda=\sum_{i \in I} x_{i} \lambda f_{i} .
$$

Given $0 \neq \lambda \in D$, let $\tilde{\lambda}: V \rightarrow V$ be the linear transformation sending $v$ to $v \lambda$. Then $w(\lambda) \geq g \Rightarrow \tilde{\lambda} w \geq g$.

This was established in $\S 2$, Theorem 2.7 , point 2 . inequality (27)'.

Claim 3. For $0 \neq \lambda \in D, i, j \in I$ fixed let $\phi^{(i, j)}: V \rightarrow V$ be the linear transformation sending $v=\sum x_{\iota} f_{\iota}$ onto $v \phi_{\lambda}^{(i, j)}=x_{i} \lambda f_{j}$. Then $\operatorname{Mtrx}\left(\phi_{\lambda}^{(i, j)}\right)$ $=E^{(i, j)} \lambda$, and $\phi_{\lambda}^{(i, j)} \in L_{0}$ (resp. $\phi^{(i, j)} \in L_{0}^{+}$) as soon as $\lambda \in J^{w_{i j}}$ (resp. $\left.\lambda \in J^{w_{i \jmath}^{+}}\right)$.

That the matrix of $\phi_{\lambda}^{(i, j)}$ is the standard $(i, j)$ matrix $E^{(i, j)}$ times $\lambda$ this is formal. Choose $\lambda \in J^{w_{i}}$ (resp. $\lambda \in J^{w_{i j}^{+}}$). The assertion is equivalent to $\phi_{\lambda}^{(i, j)}$ increases (strictly increases) lengths. Let then $0 \neq v \in V$. If $v=$ $\sum_{\iota \in I} x_{i} f_{\iota}$ then

$$
w(v \cdot v)=w\left(\sum_{k \in I} x_{k} s_{k} x_{k}^{*}\right)=\underset{k \in I}{\operatorname{Min}} w\left(x_{k} s_{k} x_{k}^{*}\right) \leq w\left(x_{i} s_{i} x_{i}^{*}\right)
$$




$$
\begin{aligned}
& w\left(v^{(i, j)} \cdot v^{(i, j)}\right)=w\left(x_{i} \lambda f_{j} \cdot x_{i} \lambda f_{j}\right) \\
& \quad=w\left(x_{i} \lambda\left(f_{j} \cdot f_{j}\right)\left(x_{i} \lambda\right)^{*}\right)=2 w\left(x_{i}\right)+2 w(\lambda)+w\left(s_{j}\right) \\
& \quad=w\left(x_{i} s_{i} x_{i}^{*}\right)+2 w(\lambda)+w\left(s_{j}\right)-w\left(s_{i}\right) \\
& \quad=w\left(x_{i} s_{i} x_{i}^{*}\right)+2 w(\lambda)+w_{j i} \geq w(v \cdot v)+\left(2 w(\lambda)-w_{i j}\right) .
\end{aligned}
$$

From the preceding inequality all that is left to show is that $2 w(\lambda)-$ $w_{i j} \geq 0$ (resp. $2 w(\lambda)-w_{i j}>0$ ). Now, in the case $\lambda \in J^{w_{i j}}, w_{i j} \leq 0$, then

$$
2 w(\lambda)-w_{i j} \geq 2 w(\lambda) \geq 0 ;
$$

in the case $\lambda \in J^{w_{i}}, w_{i j}>0$, then $w(\lambda) \geq \varepsilon$ so that

$$
2 w(\lambda)-w_{i j} \geq 2 \varepsilon-\varepsilon=\varepsilon>0 .
$$

This shows that $\phi^{(i, j)}$ increases lengths. In the case $\lambda \in J^{w_{i j}^{+}}$when $w_{i j}=0$, $2 w(\lambda)-w_{i j}=2 w(\lambda)$ follows and then $w_{i j}^{+}=\varepsilon$ so that $w(\lambda) \geq \varepsilon$, whence $2 w(\lambda)-w_{i j}>0$; when $w_{i j}=-\varepsilon$ then $2 w(\lambda)-w_{i j}=2 w(\lambda)+\varepsilon \geq \varepsilon>0$; when $w_{i j}=\varepsilon$, then $2 w(\lambda)-w_{i j} \geq \varepsilon>0$. This shows that $\phi^{(i, j)}$ strictly increases lengths, as desired.

Claim 4. $R_{W} \subset \operatorname{Mtrx}\left(L_{0}\right)$ so that $R_{W}=\operatorname{Mtrx}\left(L_{0}\right)$.

Let $\phi \in R_{W}$. There is $\phi \in L$ with $\Phi=\operatorname{Mtrx}(\phi)$. It is to be shown that $\phi \in L_{0}$ or, it is the same, $\phi$ increases lengths. Put $v=\sum_{\iota} x_{i} f_{\imath}$. The equation $v \phi=v^{\prime}, v^{\prime} \in V$ translated into matrix equation is now $\underline{v}=\underline{v}^{\prime}$. Since $\underline{v}^{\prime}$ is nullary vector over $D$ there is a finite set $I_{1}$ of $I$ such that if $\Phi^{\prime}=\sum_{i, j \in I} E^{(i, j)} \phi_{i j}$ then $\underline{v} \Phi^{\prime}=\underline{v}^{\prime}$. By construction, $\phi_{i j} \in J^{w_{\imath j}}, i, j \in I_{1}$. If $\phi^{(i, j)}=\operatorname{Mtrx}^{-1}\left(E_{\phi_{i j}}^{(i, j)}\right)$ then $\phi^{(i, j)}=\phi_{\lambda_{t, j}}^{(i, j)}$, where $\lambda_{i j}=\phi_{\imath j} \in J^{w_{\imath}}$. By the preceding claim, $\phi^{(i, j)} \in L_{0}$. If $\phi^{\prime}=\operatorname{Mtrx}^{-1}\left(\Phi^{\prime}\right)$ then $\phi^{\prime}=\sum_{i, j \in I_{1}} \phi_{\lambda_{t, j}}^{(i, j)} \in$ $L_{0}$. Since $v=v \phi^{\prime}$ it follows that $w(v \phi \cdot v \phi)=w\left(v \phi^{\prime} \cdot v \phi^{\prime}\right) \geq w(v \cdot v)$, for every $v \in V$, as desired.

\section{Claim 5. $J_{W}=\operatorname{Mtrx}\left(L_{0}^{+}\right)$.}

For the inclusion $J_{W} \subset \operatorname{Mtrx}\left(L_{0}^{+}\right)$use a similar argument as in the preceding claim based on claim 3. For the inclusion $\operatorname{Mtrx}\left(L_{0}^{+}\right) \subset J_{W}$ proceed as follows. From $\phi \in L_{0}^{+}$follows

$$
2 w\left(\phi_{i j}\right)>w\left(s_{i}\right)-w\left(s_{j}\right)=w_{\iota j} \text {. }
$$

When $w_{i j}=0$ then $w\left(\phi_{i j}\right)>0$ follows giving $w\left(\phi_{i j}\right) \geq \varepsilon$ so that $\phi_{i j} \in J=$ $J^{w_{\imath j}^{+}}$. When $w_{i j}<0$ then $\phi_{i j} \in R=J^{w_{\imath j}}=J^{w_{t j}^{+}}$. When $w_{i j}<0$ then $2 w\left(\phi_{i j}\right)$ $<w_{i j}$ implies $2 w\left(\phi_{i j}\right) \geq 2 \varepsilon$ so that $w\left(\phi_{i j}\right) \geq \varepsilon$ whence $\phi_{i j} \in J=J^{w_{\imath j}}=$ $J^{w_{\imath}^{+}}$. 
DeFinition 3.14. (Residue matrix module $W$.) Let $W$ be any $w$-matrix over $G$. Given $\Phi \in R_{I \times I}$ denote by $\bar{\Phi}_{W}$ the $I \times I$ matrix over the residue division ring $\bar{D}$ such that

$$
\begin{cases}\operatorname{entr}_{l, j}\left(\bar{\Phi}_{W}\right)=\overline{0}, & \text { if } w_{l j} \neq 0 \\ \operatorname{entr}_{i, j}\left(\bar{\Phi}_{W}\right)=\operatorname{entr}_{l, j}(\Phi)+J, & \text { if } w_{l J}=0\end{cases}
$$

I refer to $\bar{\Phi}_{W}$ as to residue matrix modulo $W$.

For instance, if $W$ is as in Illustration $(l)$, then if $\Phi=\left[\phi_{l J}\right]_{1 \leq i, J \leq 4}$, then

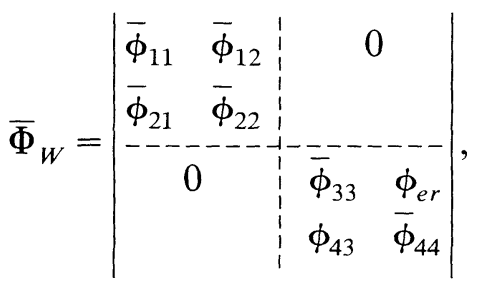

where $\bar{\phi}_{i j}=\phi_{i j}+J$. Hence,

$$
\left(\overline{R_{I \times I}}\right)_{W}=\left|\begin{array}{cc:c}
\bar{D} & \bar{D} & 0 \\
\bar{D} & \bar{D} & \\
\hdashline 0 & \frac{\bar{D}}{D} & \overline{\bar{D}}
\end{array}\right|=\bar{D}_{2 \times 2} \oplus \bar{D}_{2 \times 2}
$$

where $\oplus$ is the block diagonal sum and $\operatorname{Diag}_{2}(\bar{D})$ is the ring of $2 \times 2$ diagonal matrices over $\bar{D}(=R / J)$.

THEOREM 3.15. Let $V$ be as in Theorem 3.13. Denote by $\bar{D}_{W}$, the block diagonal sum of the ring $\bar{D}_{I_{0} \times I_{0}}$ of row-finite $I_{0} \times I_{0}$ matrices over the residue division ring $\bar{D}$, and the ring $\bar{D}_{I_{\varepsilon} \times I_{\varepsilon}}$ of row-finite $I_{\varepsilon} \times I_{\varepsilon}$ diagonal matrices over $\bar{D}$ (if $I_{\varepsilon}=\varnothing$ then discard the right summand $\operatorname{Diag}_{I_{\varepsilon}}(\bar{D})$ ). Then:

(1) $R_{W} / J_{W} \cong(R / J)_{W}=\bar{D}_{W}$.

(2) $L_{0} / L_{0}^{+} \cong \bar{D}_{W}$.

Proof. Given $\phi \in L_{0}$ define $\bar{\phi}_{W}$ to be the residue matrix modulo $W$ of the matrix of $\phi$. As $\phi$ ranges over $R_{W}$ the matrix of $\phi$ ranges over $R_{W}$ (Theorem 3.13)). Hence $\bar{\phi}_{W}$ ranges over $\bar{D}_{W}$. Now, $\bar{\phi}_{W}=0 \Leftrightarrow \operatorname{Mtrx}(\phi) \in$ $J_{W} \Leftrightarrow(\phi) \in L_{0}^{+} \quad$ (Theorem 3.13). Since $\phi \rightarrow \bar{\phi}_{W}$ is a ring homomorphism

$$
L_{0} / L_{0}^{+} \cong \bar{D}_{W}
$$

follows, which also shows that $R_{W} / J_{W} \cong \bar{D}_{W}$, as this can be checked directly. 
From the preceding theorem one can deduce several new facts about the pair $L_{0} \supset L_{0}^{+}$, some of which are of global meaning. Here are some.

COROLlaRY 3.16. (i) $L_{0}^{+}$is an intersection of maximal ideals in $L_{0}$ and, consequently, $L_{0}^{+} \supset \mathrm{Jac}\left(L_{0}\right)$. (ii) Hence, $L_{0}^{+} \cap \mathscr{F} \mathscr{L}=\operatorname{Jac}\left(L_{0}\right) \cap \mathscr{F} L$.

Proof. (i) by Theorem 3.15,

$$
\begin{aligned}
L_{0} / L_{0}^{+} & =R_{W} / J_{W}=(R / J)_{W}=\bar{D}_{W}=\bar{D}_{I_{0} \times I_{0}} \oplus \bar{D}_{I_{\varepsilon} \times I_{\varepsilon}} \\
& =\bar{D}_{I_{0} \times I_{0}} \times \bar{D}_{I_{\varepsilon} \times I_{\varepsilon}}
\end{aligned}
$$

Now $\bar{D}_{I_{0} \times I_{0}}$ is primitive and so is $\bar{D}_{I_{\varepsilon} \times I_{\varepsilon}}$. Thus $\bar{D}_{W}$ is semi-primitive. Hence $L_{0} / L_{0}^{+}$is semi-primitive. Equivalently, $L_{0}^{+}$is an intersection of maximal ideals. (ii) follows from the preceding and §2, Theorem 2.12.

COROllary 3.17. $L_{0}^{+}$is a prime ideal if, and only if $L_{0}^{+}$is primitive. Equivalently, some (and, hence, all) orthogonal basis of $V$ which is bounded by $\varepsilon$ is uniform.

Proof. If $L_{0}^{+}$is a prime ideal then $L_{0} / L_{0}^{+}$is a prime ring. Hence, $\bar{D}_{W} \cong \bar{D}_{I_{0} \times I_{0}} \times \bar{D}_{I_{\varepsilon} \times I_{\varepsilon}}$ is prime; if, and only if, $I_{\varepsilon}=\varnothing$. Equivalently, $\left(f_{i}\right)$ is uniform.

COROLlARY 3.18. $L_{0}^{+}$is a maximal ideal if and only if $V$ is finite-dimensional and every orthogonal basis of $V$ which is bounded by $\varepsilon$ is uniform.

Proof. If $L_{0}^{+}$is maximal then $\bar{D}_{W} \cong \bar{D}_{I_{0} \times I_{0}}=\bar{D}_{I \times I}$ is a simple ring. Hence, $I$ is finite so that $V$ is finite-dimensional.

Corollary 3.19. (i) If $V$ is finite-dimensional then $L_{0}^{+}=\operatorname{Jac}\left(L_{0}\right)$. (ii) Conversely, if $L_{0}^{+}=\operatorname{Jac}\left(L_{0}\right)$ and if $V$ possesses a uniform basis necessarily $V$ is finite-dimensional.

Proof. (i) follows from Corollary 3.16. (ii) From the equality $L_{0}^{+}=$ $\operatorname{Jac}\left(L_{0}\right)$ and the isomorphisms $L_{0} \cong R_{I_{0} \times I_{0}}, L_{0}^{+} \cong J_{I_{0} \times I_{0}}=\operatorname{Jac}(R)_{I_{0} \times I_{0}}$ follows the equality $\operatorname{Jac}\left(R_{I_{0} \times I_{0}}\right)=\operatorname{Jac}(R)_{I_{0} \times I_{0}}$. Since evidently $R$ contains no nilpotent elements it follows by Patterson's [14, Theorem 1] that $I_{0}$ is a finite set. Equivalently, $V$ is finite-dimensional.

Question (Open). If $L_{0}^{+}=\operatorname{Jac}\left(L_{0}\right)$ must $V$ be finite-dimensional? 
COROllary 3.20. Let $V$ be any non-degenerate 0-elliptic space with some orthogonal basis $\left(f_{i}\right)$ which is bounded by $\varepsilon$, where as usual $\varepsilon=0$ or else $\varepsilon$ is the first positive element in the value group $G$. Then the prime ring $L_{0}$ contains in its lattice of 2-sided ideals an isomorphic copy of the positive cone of $G$.

Proof. When the basis $\left(f_{i}\right)$ is uniform the result is evident. Assume to the contrary that $\left(f_{i}\right)$ is mixed so that $\varepsilon$ is the first positive element in $G$. Extend the convention about $J^{\varepsilon}$ to $J_{g}^{\varepsilon}$, specifically, put:

$$
J_{g}^{0}=J_{g}^{-\varepsilon}=J_{g}, \quad J_{g}^{\varepsilon}=J_{g+\varepsilon} \quad(g \geq 0) .
$$

Define $\left(J_{g}\right)_{W}$ (and $\left(J_{g}\right)_{W}^{+}$) similarly to $R_{W}$ (and $J_{W}$ respectively). Thus $\Phi=\left[\phi_{i j}\right] \in\left(J_{g}\right)_{W}$ if, and only if,

$$
\left\{\begin{array}{l}
\Phi \in R_{I \times I}, \\
\operatorname{entr}_{l, j}(\Phi) \cap J_{g}^{w_{l j}} \quad(\iota, j \in I) .
\end{array}\right.
$$

Exactly as for the case $g=0$ one can show that $\operatorname{Mtrx}\left(L_{g}\right)=$ $\left(J_{g}\right)_{W}\left(\operatorname{Mtrx}\left(L_{g}^{+}\right)=\left(J_{g}\right)_{W}^{+}\right)$. Since $\left(J_{g}\right)_{W}=\left(J_{g^{\prime}}\right)_{W} \Leftrightarrow g=g^{\prime}$ this makes it clear that $g \in G^{+} \rightarrow L_{g}$ is a dual isomorphism of ordered sets from $G^{+}$ into Lat. $\left(L_{0}\right)$.

Since this section began with an assumption about the unitary group $U=U(V)$ it seems appropriate to close it with information that can be derived about this group. In what will follow I will deal with a certain matrix representation of $U$ over the residue division ring $\bar{D}=R / J$, where I will assume that, again, $V$ has some orthogonal basis $\left(f_{i}\right)$, which is bounded by $\varepsilon$ with either $\varepsilon=0$ or $\varepsilon=1$ st positive element of $G$. The unitary version of Corollary 3.22 will be left to upcoming $\$ 4$.

THEOREM 3.21. Let $V$ be any non-degenerate 0-elliptic space possessing some orthogonal basis $\left(f_{\iota}\right)$, which is bounded by $\varepsilon$, where either $\varepsilon=0$ or $\varepsilon$ is the first positive element in the value group $G$. If $W$ is the w-matrix associated to $\left(f_{i}\right)$ and if $\mathscr{M}^{\times}: U \rightarrow \bar{D}_{W}$ is the mapping sending $\phi \in U$ onto $\operatorname{Mtrx}(\phi)_{W}$, the residue matrix modulo $W$ of the matrix of $\phi$ relative to the basis, then $\mathscr{M}^{\times}$is a representation of $U$ over $\bar{D}$ such that:

(1) $\operatorname{Ker}\left(\mathscr{M}^{\times}\right)=U_{0}^{+}$;

(2) $\operatorname{Im}\left(\mathscr{M}^{\times}\right)$is a subgroup of unitary matrices of the unitary group of $\bar{D}_{W}$, relative to the partial matrix involution $(\bar{S})$ of $\bar{D}_{W}$ induced by $(S)$.

Proof (1) Recall that $\sigma \rightarrow \sigma_{W}=\operatorname{Mtrx}(\sigma)_{W}$ is a ring homomorphism from $L_{0}$ onto $\bar{D}_{W}$ with kernel precisely $L_{0}^{+}$(Theorem 3.15). Since $V$ is 
0-elliptic, it follows that $U \subset L_{0}$ so that $\mathscr{M}^{\times}$is the restriction of $\sigma \rightarrow \sigma_{W}$ to $U$ and, hence, $\mathscr{M}^{\times}$is a group homomorphism from $U$ into the group of invertible matrices in $\bar{D}_{W}$. Now

$$
\operatorname{Ker}\left(\mathscr{M}^{\times}\right)=\left(1+\operatorname{Ker}\left(\sigma \rightarrow \sigma_{W}\right)\right) \cap U=\left(1+L_{0}^{+}\right) \cap U=U_{0}^{+} .
$$

(2) The ring $L_{0}$ carries a partial involution $*$ which can be identified to the partial matrix involution $(S)$ of the ring $R_{W}$. Since $J_{W}$ is $(S)$-closed it follows that $\bar{D}_{W} \cong R_{W} / J_{W}$ carries a partial involution $(S)$. By construction $\mathscr{M}^{\times}$takes $U$ onto $\mathscr{U}\left(R_{W}: S\right)$ ) and $\mathscr{U}\left(R_{W} ;(S)\right)$ is mapped onto a subgroup of $\mathscr{U}\left(\bar{D}_{W} ;(\bar{S})\right)$ under the residue homomorphism $\Phi \rightarrow \Phi_{W}$.

The following theorem is fairly formal.

THEOREM 3.22. The unitary group of the ring $\bar{D}_{W}$ relative to the induced involution $(\bar{S})$ is of the form

$$
\mathscr{U}\left(\bar{D}_{W} ;(\bar{S})\right)=\mathscr{U}\left(\bar{D}_{I_{0} \times I_{0}} ; \quad\left(\bar{S}_{0}\right)\right) \times \mathscr{U}\left(\bar{D}_{I_{\varepsilon} \times I_{\varepsilon}} ;\left(\bar{S}_{\varepsilon}\right)\right),
$$

where $\left(\bar{S}_{0}\right)$ takes $\bar{\Phi}_{(0)}=\left[\phi_{i j}+J\right]_{i, j \in I_{0}}$ onto $\bar{\Phi}_{(0)}^{\left(S_{0}\right)}=\left[s_{i} \phi_{j, i}^{*} s_{j}^{-1}+J\right]_{i, j \in I_{0}}$ (when $\bar{\Phi}_{0}$ is both row and column finite) and, similarly, $\left(\bar{S}_{\varepsilon}\right)$ takes $\Phi_{(1)}=$ $\left[\phi_{i, j}^{*}+J\right]_{i, j \in I_{\varepsilon}}$ onto $\bar{\Phi}_{(1)}^{\left(S_{\varepsilon}\right)}=\left[s_{i} \phi_{j, i}^{*} s_{J}^{-1}+J\right]_{i, j \in I_{\varepsilon}}$.

THEOREM 3.23. Let $V$ be any non-degenerate 0-elliptic space and suppose that the $R$-module $V_{0}$ has an orthogonal basis $\left(f_{\iota}\right)$ all of whose members are medial vectors. If, further, $1 / 2 \in R$, then the normal subgroup $\mathscr{C} \mathscr{F}(U)$ of unitary transformations $\phi$ such that $\phi-1$ is of finite rank maps onto the corresponding subgroup $\mathscr{C} \mathscr{F}(\bar{U})$ of $\bar{D}$.

Proof. It is to be shown first that the group homomorphism $\phi \in U \rightarrow$ $\bar{\phi} \in \bar{U}$ extends to an onto homomorphism $\sigma \in L_{0} \rightarrow \bar{\sigma} \in \bar{L}=L(\bar{V})$. For if $\sigma \in L_{0}$ it is clear that $V_{0} \sigma \subset V_{0}$ and $V_{0}^{+} \sigma \subset V_{0}^{+}$. Hence if

$$
\overline{\boldsymbol{\sigma}}=v+V_{0}^{+} \rightarrow v \boldsymbol{\sigma}+V_{0}^{+}
$$

then $\sigma \rightarrow \bar{\sigma}$ is a ring homomorphism from $L_{0}$ into $\bar{L}$ with correspondence of the adjoint involution in $L_{0}$ and $\bar{L}$ respectively. To show that $\sigma \rightarrow \bar{\sigma}$ is onto proceed as follows. If $\bar{\sigma} \in \bar{L}$ then using the basis $\left(\bar{f}_{i}\right)=\left(f_{i}+V_{0}^{+}\right)$of $\bar{V}$ lift $\bar{\sigma}$ to a linear transformation $\sigma_{0}$ of the $R$-module $V_{0}$ sending $f_{i}$ onto $\bar{f}_{i} \bar{\sigma}$. By construction $\sigma_{0}$ maps $V_{0}^{+}$into $V_{0}^{+}$. Extend $\sigma_{0}$ to a linear transformation $\sigma$ of $V$ by the rule

$$
(\lambda v) \sigma=\lambda\left(v \sigma_{0}\right) \quad\left(\lambda \in D, v \in V_{0}\right) .
$$


Given any $u \in V$, there is $\lambda_{0} \in D$ such that $\lambda_{0} u$ is medial ( $\$ 1$, Theorem 1.7). Then $\left(\lambda_{0} u\right) \sigma \in V_{0}$ so that

$$
w\left(\left(\lambda_{0} u\right) \sigma \cdot\left(\lambda_{0} u\right) \sigma\right) \geq 0
$$

or,

$$
w(u \sigma \cdot u \sigma) \geq-2 w\left(\lambda_{0}\right)=w(u \cdot u) .
$$

Thus $\sigma \in L_{0}$. Hence $\sigma \rightarrow \bar{\sigma}$ maps $L_{0}$ onto $\bar{L}$, as desired.

Next I will show that each $\phi \in \mathscr{C} \mathscr{F}(U)$ maps onto $\bar{\phi} \in \mathscr{C} \mathscr{F}(\bar{U})$. Indeed, $\phi$ maps onto $\bar{\phi} \in \bar{U}$. To say that $\phi \in \mathscr{C} \mathscr{F}(U)$ is to say $\phi-1$ is of finite rank. Equivalently, $\operatorname{Mtrx}(\phi-1)$ has all its entries zeros but for a finite subset of $I \times I$ ( finite matrix). Relative to the induced basis $\left(\bar{f}_{i}\right)$ it is clear that $\operatorname{Mtrx}(\bar{\phi}-1)$ is, again, co-finite. Thus $\bar{\phi} \in \mathscr{C} \mathscr{F}(\bar{U})$.

It remains to show that each $\bar{\phi} \in \mathscr{C} \mathscr{F}(\bar{U})$ lifts to a unitary transformation $\phi \in \mathscr{C} \mathscr{F}(U)$.

Let $\bar{V}^{(1)}$ be the image of $\bar{\phi}-1$. By construction, $\bar{V}^{(1)}$ is a finite-dimensional subspace of $\bar{V}$. By $\S 1$ Theorem $1.11, \bar{V}^{(1)}$ lifts to a finite-dimensional orthogonal summand $V_{0}^{(1)}$ of $V_{0}$. Denote by $\bar{V}^{(2)}$ the orthogonal complement of $\bar{V}^{(1)}$ and by $V_{0}^{(2)}$ the orthogonal complement of $V_{0}^{(1)}$ in $V_{0}$. By the cited theorem $V_{0}^{(2)}$ maps onto $\bar{V}^{(2)}$. I distinguish two cases.

Case $\bar{\phi}$ is a symmetry. Here $\bar{\phi}^{2}=\bar{\phi}$ implies

$$
\bar{\phi}=-1_{\bar{V}^{(1)}} \oplus 1_{\bar{V}^{(2)}} \text {. }
$$

If $\phi_{0}=-1_{V_{0}^{(1)}} \oplus 1_{V_{0}^{(2)}}$ then $\phi_{0}$ extends in a unique way to the symmetry $\phi$ of $V$. Hence $\bar{\phi}$ has been lifted to the symmetry $\phi$ in $L_{0}$. By construction, $\phi$ is co-finite.

Case $1+\bar{\phi}$ is non-singular. Here, $\bar{\phi}$ can be Cayley parametrized. If $\bar{\tau}=(1-\bar{\phi}) /(1+\bar{\phi})$, then

$$
\bar{V} \bar{\tau}=\bar{V}\left(\frac{1}{1+\bar{\phi}}\right)(1-\bar{\phi})=\bar{V}(1-\bar{\phi})=\bar{V}^{(1)} .
$$

Then $\bar{\tau}=\bar{\tau} / \bar{V}^{(1)} \oplus 0_{\bar{V}^{(2)}}$. Repeating the argument to the effect $L_{0}(V)$ maps onto $L(\bar{V})$ for $V$ replaced by $D V_{0}^{(1)}$ one readily gets that $\bar{\tau} / V^{(1)}$ lifts to a skew-symmetric transformation $\tau_{1}$ of $D V_{0}^{(1)}$. If $\tau=\tau_{1} \oplus 0$ then $\tau=-\tau^{*} \in L_{0}$ maps onto $\bar{\tau}$. By construction, $V \tau=D V_{0}^{(1)}$ is finite-dimensional. If $\phi=(1-\tau) /(1+\tau)$ then $\phi \in U$ maps onto $\bar{\phi}$ and

$$
V(1-\phi)=V\left(\frac{1}{1+\tau}\right)(-2 \tau)=V(2 \tau)=V \tau=D V_{0}^{(1)}
$$

Thus $\phi$ is co-finite. 
For general $\bar{\phi} \in \bar{U}$, by standard argument $\bar{\phi}=\bar{\phi}_{1} \bar{\phi}_{2}$, where $\bar{\phi}_{1}$ is a symmetry and $\bar{\phi}_{2}$ is such that $1+\bar{\phi}_{2}$ is non-singular. One can, further, choose both $\bar{\phi}_{1}, \bar{\phi}_{2}$ to be co-finite. It suffices then to lift separately $\bar{\phi}_{1}, \bar{\phi}_{2}$ to $\phi_{1}, \phi_{2}$ co-finite. Then $\bar{\phi}$ is lifted to the co-finite unitary $\phi_{1}, \phi_{2}$.

Corollary 3.24. If $V$ is as in Theorem 3.23 then $M^{\times}: U \rightarrow \bar{D}_{W}$ is a matrix representation of $U$ over the residue division ring $\bar{D}=R / J$ with the following features.

(1) $\operatorname{Ker}\left(M^{\times}\right)=U_{0}^{+}$

(2) If $M^{\mathscr{C F}}$ is the restriction of $M^{\times}$to the normal subgroup $\mathscr{C} \mathscr{F}(U)$ of co-finite unitary transformations in $U$ then

$$
\operatorname{Ker}\left(M^{\mathscr{C F}}\right)=\mathscr{C} \mathscr{F}(U) \cap\left(1+\operatorname{Jac}\left(L_{0}\right)\right) .
$$

(3) $\mathscr{C} \mathscr{F}(U)$ maps onto $\mathscr{C} \mathscr{F}\left(\bar{D}_{I_{0} \times I_{0}} ;\left(\bar{S}_{0}\right)\right)$, the normal subgroup of unitary matrices $\bar{\Phi}$ in $\bar{D}_{I_{0} \times I_{0}}$ such that $\bar{\phi}-1$ is a finite matrix.

Question. (Open.) What is the status of Corollary 3.24 in the case of mixed basis? Also, if $U$ maps onto the unitary group of $\bar{D}_{W}$ must $V$ be finite-dimensional?

4. Normal subgroups of the projective group. Let $V$ be any finitedimensional non-degenerate elliptic space. Denote by $U^{(0)}$ the unitary group, $U$, by $U^{(1)}=[U, U]$, the derived unitary group and by $U^{(r)}$, the $r$ th derived unitary group

$$
U^{(r)}=\left[U^{(r-1)}, U^{(r-1)}\right] \quad(r \geq 1) .
$$

Let $P U^{(r)}$ stand for the factor group $U^{(r)} / U^{(r)} \cap Z$. I refer to $P U^{(1)}$ as to the projective derived unitary group, in short, the projective group of $V$. Let lat. $\left(P U^{(r)}\right)$ stand for the lattice of normal subgroups of the group $P U^{(r)}$.

Given $g \in G$, associate to $g$ the normal subgroup $U_{g}$ of $U$. To $U_{g}$ and $r \geq 0$ correspond the normal subgroup $U_{g}^{(r)}=\left[U_{g}^{(r-1)}, U_{g}^{(r-1)}\right]$ if $r \geq 1$ and $U_{g}^{(0)}=U_{g}$. To $U_{g}^{(r)}$ corresponds the normal subgroup $P U_{g}^{(r)}=$ $U_{g}^{(r)} / U^{(r)} \cap Z$ of $P U^{(r)}$. This section is concerned with the nature of the mapping $g \rightarrow P U_{g}^{(r)}$, where $g$ ranges over a suitable open segment of $G$. Of particular importance is the special case $r=1$. The work is organized as follows.

4.1: Equalizers of the mapping $g \rightarrow U_{g}$ and of related mappings.

4.2: The mapping $g \rightarrow P U_{g}^{(1)}$ for $\operatorname{dim}_{D} V>1$.

4.3: The mapping $g \rightarrow P U_{g}^{(1)}$ for $\operatorname{dim}_{D} V=1$.

4.4: The general mapping $g \rightarrow P U_{g}^{(r)}$. 


\subsection{Equalizers of the mapping $g \rightarrow U_{g}$ and of related mappings.}

Throughout the rest of this section the mapping $g \rightarrow U_{g}$ and the derived mappings $g \rightarrow P U_{g}^{(r)}$, will all be restricted to the open segment $(w(2), \rightarrow)$ of $G$. To simplify the writing let me make the

Definition 4.1.1. (1) The mapping $g \in(w(2), \rightarrow) \rightarrow U_{g}$ from $(w(2), \rightarrow)$ into lat. $(U)$ is denoted by $\pi_{0}$

(2) The mapping $g \in(w(2), \rightarrow) \rightarrow P U_{g}^{(r)} \in$ lat. $\left(P U^{(r)}\right)$ is denoted by $P \pi_{r}$. $P \pi_{r}$.

(3) The mapping $g \in(w(2), \rightarrow) \rightarrow U_{g}^{(r)} \in$ lat. $\left(U^{(r)}\right)$ is denoted by

The equalizer of, say, $\pi_{0}$ is the subset of pairs $\left(g_{1}, g_{2}\right)$ such that $\pi_{0}\left(g_{1}\right)=\pi_{0}\left(g_{2}\right)$. Facts about the equalizers of $\pi_{0}$ and $\pi_{1}$ will be collected in this subsection with a view to apply the results to subsections 4.2 and 4.3. One more mapping is in order.

Definition 4.1.2. Let $\Sigma_{0}:(w(2), \rightarrow) \rightarrow$ lat. $((V))$ be the mapping sending $g \in(w(2), \rightarrow)$ onto the additive subgroup

$$
\mathscr{K}_{g}\left(=\left\{\phi=-\phi^{*} \in L \mid \phi w \geq g\right\}\right) .
$$

As observed and established in $\S 2$, Theorem 2.16:

Facts 4.1.3. (a) Let $\mathscr{C}$ be the partial operator of $L=L(V)$ which is defined at $\phi \in L$ if and only if $1+\phi$ is invertible and then

$$
\mathscr{C}(\phi)=2(1-\phi) /(1+\phi) \text {. }
$$

Let $\mathscr{C}^{-1}$ be the partial operator of $L$ which is defined at $\phi \in L$ if and only if $1+\sigma / 2$ is invertible and then

$$
\mathscr{C}^{-1}(\sigma)=(1-\sigma / 2) /(1+\sigma / 2) .
$$

Then $\mathscr{C}, \mathscr{C}^{-1}$ are one-to-one and $\mathscr{C} \circ \mathscr{C}^{-1}=1_{L}=\mathscr{C}^{-1} \circ \mathscr{C}$

(b) $\mathscr{C}$ is entirely defined at $\pi_{0}(g)$ and $\mathscr{C}\left(\pi_{0}(g)\right)=\Sigma_{0}(g)$ for every $g>w(2)$

(c) $\mathscr{C}^{-1}$ is entirely defined at $\Sigma_{0}(g)$ and $\mathscr{C}^{-1}\left(\Sigma_{0}(g)\right)=\pi_{0}(g)$ for every $g>w(2)$.

Fact 4.1.4. $\pi_{0}$ as well as the derived mappings $\pi_{1}, p \pi_{0}, p \pi_{1}, \ldots$ are dual order homomorphisms of ordered sets.

Facts 4.1.3, points (b) and (c) tell us that $\pi_{0}$ factors through $\mathscr{C}^{-1}$, $\pi_{0}=\mathscr{C}^{-1} \circ \Sigma_{0}$. Hence $\pi_{0}$ and $\Sigma_{0}$ have the same equalizers. 
Question. (Open.) If $\Sigma_{1}:(w(2), \rightarrow) \rightarrow$ lat $(\mathscr{K})$ is the mapping sending $g$ onto $\left(\Sigma_{0}(g), \Sigma_{0}(g)\right)$, the Lie commutator subgroup of $\Sigma_{0}(g)$ and if $\pi_{1}=g \in(w(2), \rightarrow) \rightarrow\left[\pi_{0}(g), \pi_{0}(g)\right]$, the commutator subgroup of the unitary subgroup $\pi_{0}(g)$, does it follow that $\pi_{1}=\mathscr{C}^{-1} \circ \Sigma_{1}$ ? that $\pi_{1}$ and $\Sigma_{1}$ have same equalizers?

I do not know general answers to the preceding questions. Some partial results tending to affirmative answers will be established.

Recall that if $M$ is the set of medial transformations $\phi$ of $V$ such that $\phi^{*}$ exists and $\phi^{*}$ is medial then $M$ is a group (medial group of $V$ ) all of whose members $\phi$ are such that

$$
\phi L_{g}=L_{g} \phi f=L_{g} \quad(g \in G) .
$$

Equivalently,

$$
\psi \in L \Leftrightarrow g \in G ; \quad \psi w \geq g \Leftrightarrow \phi \psi w \geq g \Leftrightarrow \psi \phi w \geq g .
$$

Definition 4.1.5. I will say that $\phi_{1}, \phi_{2} \in L=L(V)$ are equivalent (notation: $\phi_{1} \equiv \phi_{2}$ ) if

$$
\phi_{2}=\mu_{1} \phi_{1} \mu_{2}
$$

for some $\mu_{i} \in M(i=1,2)$.

As a corollary to the preceding definition:

Fact 4.1.6. If $\phi_{1} \equiv \phi_{2}$ then for every $g \in G, \phi_{1} w \geq g \Leftrightarrow \phi_{2} w \geq g$.

Fact 4.1.7. If $\sigma, \sigma^{\prime} \in \pi_{0}(g)$, then for $\left[\sigma, \sigma^{\prime}\right]=\sigma^{-1} \sigma^{\prime-1} \sigma \sigma^{\prime}$ and for $\left(\phi, \phi^{\prime}\right)=\phi \phi^{\prime}-\phi^{\prime} \phi$ where $\phi=\mathscr{C}(\sigma), \phi^{\prime}=\mathscr{C}\left(\sigma^{\prime}\right)$ :

(1) $\mathscr{C}\left(\left[\sigma, \sigma^{\prime}\right]\right) \equiv\left[\sigma, \sigma^{\prime}\right]-1$

(2) $\left[\sigma, \sigma^{\prime}\right]-1 \equiv\left(\phi, \phi^{\prime}\right)$.

Proof. (1) I will show more generally that if $\sigma \in \pi_{0}(g)$, then $\sigma-1 \equiv$ $\mathscr{C}(\sigma)$ or, $\sigma-1 \equiv \phi$, where $\phi=\mathscr{C}(\sigma)$. By construction,

$$
\sigma-1=\mathscr{C}^{-1}(\phi)-1=\frac{1-\phi / 2}{1+\phi / 2}-1=\frac{-\phi}{1+\phi / 2} .
$$

By construction, $g>w(2)$. From $\sigma \in \pi_{0}(g)$ follows $\phi=\mathscr{C}(\sigma) \in \Sigma_{0}(g)$ and, hence, $\phi \in L_{g}$ so that $\phi / 2 \in L_{g-g(2)} \subset L_{0}^{+}$. From $\S 2$, Theorem 2.10 follows that $1+\phi / 2$ is medial. Since $\phi / 2$ has evidently adjoint which must be in $L_{0}^{+}$it follows that $(1+\phi / 2) *$ is medial. Thus $1+\phi / 2 \in M$. Then

$$
\sigma-1=\left(\frac{-1}{1+\phi / 2}\right) \phi
$$


with $-1 /(1+\phi / 2) \in M$ for $-1 \in M$ and $1+\phi / 2 \in M$. Hence, $\sigma-1$ $\equiv \phi$.

(2)

$$
\begin{aligned}
& {\left[\sigma, \sigma^{\prime}\right]-1=\sigma^{-1} \sigma^{\prime-1}\left(\sigma, \sigma^{\prime}\right) \equiv\left(\sigma, \sigma^{\prime}\right) \quad(U \subset M)} \\
& =\left(\mathscr{C}^{-1}(\phi), \mathscr{C}^{-1}(\phi)\right)=\left(\frac{1-\phi / 2}{1+\phi / 2}, \frac{1-\phi^{\prime} / 2}{1+\phi^{\prime} / 2}\right) \\
& =\left(-1+\frac{2}{1+\phi / 2},-1+\frac{2}{1+\phi^{\prime} / 2}\right) \\
& =4\left(\frac{1}{1+\phi / 2}, \frac{1}{1+\phi^{\prime} / 2}\right) \\
& =4 \frac{1}{1+\phi / 2}\left(\frac{1}{1+\phi^{\prime} / 2}, \frac{\phi}{2}\right) \frac{1}{1+\phi / 2} \\
& =4 \frac{1}{1+\phi / 2}\left(\frac{1}{1+\phi^{\prime} / 2}, \phi\right) \frac{1}{1+\phi / 2} \\
& =2 \frac{1}{1+\phi / 2}\left(\frac{1}{1+\phi^{\prime} / 2}, \phi\right) \frac{1}{1+\phi / 2} \\
& =2 \frac{1}{1+\phi / 2} \frac{1}{1+\phi^{\prime} / 2}\left(\phi, \frac{1+\phi^{\prime}}{2}\right) \frac{1}{1+\phi / 2} \frac{1}{1+\phi^{\prime} / 2} \\
& =\frac{1}{1+\phi / 2} \frac{1}{1+\phi^{\prime} / 2}\left(\phi, \phi^{\prime}\right) \frac{1}{1+\phi^{\prime} / 2} \frac{1}{1+\phi / 2} .
\end{aligned}
$$

As before, from $\phi, \phi^{\prime} \in \mathscr{K}_{g}, g>w(2)$, follow $1+\phi / 2,1+\phi^{\prime} / 2 \in M$, so that

$$
\left(\sigma, \sigma^{\prime}\right) \equiv\left(\phi, \phi^{\prime}\right)
$$

Thus

$$
\left[\sigma, \sigma^{\prime}\right]-1 \equiv\left(\sigma, \sigma^{\prime}\right) \equiv\left(\phi, \phi^{\prime}\right)
$$

as desired.

Fact 4.1.8. Let $\left(g_{1}, g_{2}\right)$ be in the equalizer of $\pi_{1}$ (e.g. $\left[U_{g_{1}}, U_{g_{1}}\right]=$ $\left.\left[U_{g_{2}}, U_{g_{2}}\right]\right)$. If $\Sigma_{1}=g \in(w(2), \rightarrow) \rightarrow\left(\Sigma_{0}(g), \Sigma_{0}(g)\right)\left(=\left(\mathscr{K}_{g}, \mathscr{K}_{g}\right)\right)$ then

$$
\Sigma_{1}\left(g_{1}\right) \subset \Sigma_{0}\left(2 g_{2}\right) ; \quad \Sigma_{1}\left(g_{2}\right) \subset \Sigma_{0}\left(2 g_{1}\right) \text {. }
$$

Proof. Let $\phi, \phi^{\prime} \in \Sigma_{0}\left(g_{1}\right)$. If $\sigma, \sigma^{\prime}=\mathscr{C}^{-1}(\phi), \mathscr{C}^{-1}\left(\phi^{\prime}\right)$ then $\sigma, \sigma^{\prime} \in$ $\pi_{0}\left(g_{1}\right)$. Thus $\left[\sigma, \sigma^{\prime}\right] \in \pi_{0}\left(g_{1}\right) \in \pi_{0}\left(g_{1}\right)=\pi_{1}\left(g_{2}\right)=\left[U_{g_{2}}, U_{g_{2}}\right] \subset U_{2 g_{2}}$. (§2, Theorem 2.14, (iii)). Equivalently, $\left[\sigma, \sigma^{\prime}\right]-1 w \geq 2 g_{2}$. By Fact 4.1.7, 
$\left(\phi, \phi^{\prime}\right) \equiv\left(\left[\sigma, \sigma^{\prime}\right]-1\right) w \geq 2 g_{2}$ giving $\left(\phi, \phi^{\prime}\right) w \geq 2 g_{2}$, so that $\left(\phi, \phi^{\prime}\right) \in$ $\Sigma_{0}\left(2 g_{2}\right)$. As this holds for every pair $\phi, \phi^{\prime} \in \Sigma_{0}\left(g_{1}\right)$, it follows that $\Sigma_{1}\left(g_{1}\right) \subset \Sigma_{0}\left(2 g_{2}\right)$. By symmetry, $\Sigma_{1}\left(g_{2}\right) \subset \Sigma_{0}\left(2 g_{1}\right)$.

Fact 4.1.9. Let $\sigma, \sigma^{\prime} \in U_{w(2)}^{+}$. If

$$
\gamma=\left[\left[\sigma, \sigma^{\prime}\right], \sigma^{\prime}\right]
$$

and if $\phi, \phi^{\prime}=\mathscr{C}(\sigma), \mathscr{C}\left(\sigma^{\prime}\right)$, then

$$
\begin{aligned}
\gamma-1 \equiv & \mu_{1}\left(\phi, \phi^{\prime}\right) \mu_{2}\left(\phi, \phi^{\prime}\right) \mu_{3}+\mu_{4}\left(\phi, \phi^{\prime}\right) \mu_{5}\left(\phi, \phi^{\prime}\right) \mu_{6} \\
& +\mu_{7}\left(\left(\phi, \phi^{\prime}\right), \phi^{\prime}\right) \mu_{8}
\end{aligned}
$$

for some $\mu_{i} \in M$.

Proof. Since $\left[\sigma, \sigma^{\prime}\right] \in\left[U_{w(2)}^{+}, U_{w(2)}^{+}\right] \subset U_{w(2)}^{+}$it follows that

$$
\left[\sigma, \sigma^{\prime}\right]=\mathscr{C}^{-1}\left(\mathscr{C}\left(\left[\sigma, \sigma^{\prime}\right]\right)\right)=\mathscr{C}^{-1}(t)=\frac{1-t / 2}{1+t / 2},
$$

where $t / 2=\left(1-\left[\sigma, \sigma^{\prime}\right]\right) /\left(1+\left[\sigma, \sigma^{\prime}\right]\right)$. If $t_{1}=1-\left[\sigma, \sigma^{\prime}\right]$, then

$$
t / 2=\frac{t_{1}}{2-t_{1}} .
$$

From Fact 4.1.7 applied to [ $\left.\sigma, \sigma^{\prime}\right]$ and $\sigma^{\prime}$ follows

$$
\gamma=\left[\left[\sigma, \sigma^{\prime}\right], \sigma^{\prime}\right]-1 \equiv\left(t, \phi^{\prime}\right),
$$

where

$$
\phi, \phi^{\prime}=\mathscr{C}(\sigma), \quad \mathscr{C}\left(\sigma^{\prime}\right)=2 \frac{(1-\sigma)}{(1+\sigma)}, \quad 2 \frac{\left(1-\sigma^{\prime}\right)}{\left(1+\sigma^{\prime}\right)}
$$

Now,

$$
\begin{aligned}
\left(t, \phi^{\prime}\right) & =\left(2 \frac{t_{1}}{2-t_{1}}, \phi^{\prime}\right)=2\left(\frac{t_{1}}{2-t_{1}}, \phi^{\prime}\right) \\
& =2\left(-1+\frac{2}{2-t_{1}}, \phi^{\prime}\right)=2\left(\frac{2}{2-t_{1}}, \phi^{\prime}\right) \\
& =4 \frac{1}{2-t_{1}}\left(\phi^{\prime}, 2-t_{1}\right) \frac{1}{2-t_{1}}=4 \frac{1}{2-t_{1}}\left(t_{1}, \phi^{\prime}\right) \frac{1}{2-t_{1}} \\
& =4 \frac{1}{2\left(1-t_{1} / 2\right)}\left(t_{1}, \phi^{\prime}\right) 2\left(\frac{1-t_{1}}{2}\right) \\
& =\frac{1}{1-t_{1} / 2}\left(t_{1}, \phi^{\prime}\right) \frac{1}{1-t_{1} / 2} .
\end{aligned}
$$


From $t_{1}=1-\left[\sigma, \sigma^{\prime}\right]$ and $\left[\sigma, \sigma^{\prime}\right] \in U_{w(2)}^{+}$follows $t_{1} / 2 \in L_{0}^{+}$. Since $t_{1} / 2$ has adjoint it follows that $1-t_{1} / 2 \in M$, giving

$$
\gamma \equiv\left(t, \phi^{\prime}\right) \equiv\left(t_{1}, \phi^{\prime}\right) \text {. }
$$

Here,

$$
\begin{aligned}
\left(t_{1}, \phi^{\prime}\right) & =\left(1-\left[\sigma, \sigma^{\prime}\right], \phi^{\prime}\right) \\
& =\left(\phi^{\prime},\left[\sigma, \sigma^{\prime}\right]-1\right)=\left(\phi^{\prime}, \sigma^{-1} \sigma^{\prime-1}\left(\sigma, \sigma^{\prime}\right)\right) \\
& =\left(\phi^{\prime}, \sigma^{-1} \sigma^{\prime-1}\right)\left(\sigma, \sigma^{\prime}\right)+\sigma^{-1} \sigma^{\prime-1}\left(\phi^{\prime},\left(\sigma, \sigma^{\prime}\right)\right) .
\end{aligned}
$$

Since $\phi^{\prime}$ evidently commutes with $\sigma^{\prime-1}$ it follows that

$$
\left(t_{1}, \phi^{\prime}\right)=\left(\phi^{\prime}, \sigma^{-1}\right) \sigma^{\prime-}\left(\sigma, \sigma^{\prime}\right)+\sigma^{-1} \sigma^{\prime-1}\left(\phi^{\prime},\left(\sigma, \sigma^{\prime}\right)\right)=\tau_{1}+\tau_{2},
$$

where $\tau_{1}=\left(\phi^{\prime}, \sigma^{-1}\right) \sigma^{\prime-1}\left(\sigma, \sigma^{\prime}\right)$ and $\tau_{2}=\sigma^{-1} \sigma^{\prime-1}\left(\phi^{\prime},\left(\sigma, \sigma^{\prime}\right)\right)$.

Now,

$$
\begin{aligned}
\left(\phi^{\prime}, \sigma^{-1}\right) & =\left(\phi^{\prime}, \frac{1+\phi / 2}{1-\phi / 2}\right)=\left(\phi^{\prime},-1+\frac{2}{1-\phi / 2}\right) \\
& =\left(\phi^{\prime}, \frac{2}{1-\phi / 2}\right)=2 \frac{1}{1-\phi / 2}\left(1-\phi / 2, \phi^{\prime}\right) \frac{1}{1-\phi / 2} \\
& =\frac{1}{1-\phi / 2}\left(\phi^{\prime}, \phi\right) \frac{1}{1-\phi / 2} \equiv\left(\phi^{\prime}, \phi\right) .
\end{aligned}
$$

From Fact $4.1 .7,\left(\sigma, \sigma^{\prime}\right) \equiv\left(\phi, \phi^{\prime}\right)$ follows. Thus

$$
\tau_{1}=\frac{1}{1-\phi / 2}\left(\phi^{\prime}, \phi\right) \frac{1}{1-\phi / 2} \sigma^{\prime-1} \mu_{1}^{\prime}\left(\phi, \phi^{\prime}\right) \mu_{2}^{\prime}
$$

where $\mu_{1}^{\prime}, \mu_{2}^{\prime} \in M$. Since $1 /(1-\varepsilon / 2)$ and $\sigma^{\prime} \in M$ it follows that for some $\mu_{1}, \mu_{2}, \mu_{3} \in M$,

$$
\tau_{1}=\mu_{1}\left(\phi, \phi^{\prime}\right) \mu_{2}\left(\phi, \phi^{\prime}\right) \mu_{3} .
$$

Also,

$$
\begin{aligned}
\left(\phi^{\prime},\left(\sigma, \sigma^{\prime}\right)\right) & =\left(\phi^{\prime}, \frac{1}{1+\phi^{\prime} / 2} \frac{1}{1+\phi / 2}\left(\phi, \phi^{\prime}\right) \frac{1}{1+\phi / 2} \frac{1}{1+\phi^{\prime} / 2}\right) \\
& =\frac{1}{1+\phi^{\prime} / 2}\left(\phi^{\prime}, \frac{1}{1+\phi / 2}\left(\phi, \phi^{\prime}\right) \frac{1}{1+\phi / 2}\right) \frac{1}{1+\phi^{\prime} / 2}
\end{aligned}
$$




$$
\begin{aligned}
& +\frac{1}{1+\phi / 2}\left(\phi^{\prime},\left(\phi, \phi^{\prime}\right)\right) \frac{1}{1+\phi / 2}+\frac{1}{1+\phi / 2}\left(\phi, \phi^{\prime}\right) \\
& \cdot \frac{1}{1+\phi / 2}\left(\frac{1+\phi}{2, \phi^{\prime}}\right) \frac{1}{1+\phi / 2} \\
= & \frac{1}{2} \frac{1}{1+\phi / 2}\left(\phi, \phi^{\prime}\right) \frac{1}{1+\phi / 2}\left(\phi, \phi^{\prime}\right) \frac{1}{1+\phi / 2} \\
& +\frac{1}{2} \frac{1}{1+\phi / 2}\left(\phi, \phi^{\prime}\right) \frac{1}{1+\phi / 2}\left(\phi, \phi^{\prime}\right) \frac{1}{1+\phi / 2} \\
& +\frac{1}{1+\phi / 2}\left(\phi^{\prime},\left(\phi, \phi^{\prime}\right)\right) \frac{1}{1+\phi / 2} \\
= & \frac{1}{1+\phi / 2}\left(\phi, \phi^{\prime}\right) \frac{1}{1+\phi / 2}\left(\phi, \phi^{\prime}\right) \frac{1}{1+\phi / 2} \\
& +\frac{1}{1+\phi / 2}\left(\phi^{\prime},\left(\phi, \phi^{\prime}\right)\right) \frac{1}{1+\phi / 2} .
\end{aligned}
$$

Therefore $\left(\phi^{\prime},\left(\sigma, \sigma^{\prime}\right)\right)$ can be written in the form

$$
\left(\phi^{\prime},\left(\sigma, \sigma^{\prime}\right)\right)=\mu_{4}\left(\phi, \phi^{\prime}\right) \mu_{5}\left(\phi, \phi^{\prime}\right) \mu_{6}+\mu_{7}\left(\phi^{\prime},\left(\phi, \phi^{\prime}\right)\right) \mu_{8}
$$

where the $\mu_{i} \in M$, which completes the proof.

Recall that if $D_{n \times n}$ is the ring of $n \times n$ matrices over $D$, where $n$ is a finite integer, then if $\underline{w}: D_{n \times n} \rightarrow G^{\#}$ is the mapping sending $\Phi=$ $\left[\phi_{\iota j}\right]_{1 \leq \iota, j<n}$ onto $\underline{w}(\Phi)$ defined by

$$
\underline{w}(\Phi)=\operatorname{Min}_{i, j=1, \ldots, n}\left(w\left(\phi_{i j}\right)\right),
$$

then $\underline{w}$ is a vector valuation of $D_{n \times n}$ over $D$, which verifies the submultiplicative property

$$
\underline{w}\left(\Phi_{1} \Phi_{2}\right) \geq \underline{w}\left(\Phi_{1}\right)+\underline{w}\left(\Phi_{2}\right) \quad\left(\Phi_{1}, \Phi_{2} \in D_{n \times n}\right) .
$$

Evidently, for every $\Phi \in D_{n \times n}$ and $g \in G^{\#}$,

$$
\Phi \underline{w} \geq g \Leftrightarrow \underline{w}(\Phi) \geq g,
$$

where $\cdots \underline{w} \geq \cdots$ is the usual matrix prevaluation on $D_{n \times n}(\Phi \underline{w} \geq g$ means $w\left(\operatorname{entr}_{\iota, j}(\Phi)\right) \geq g$ for every pair $\left.i, j=1, \ldots, n\right)$. By standard argument $\underline{w}$ verifies the generalized triangle inequality

$$
\underline{w}\left(\sum_{\iota=1}^{r} \Phi_{\iota}\right) \geq \underset{i=1, \ldots, r}{\operatorname{Min}}\left(\underline{w}\left(\Phi_{i}\right)\right),
$$

and $\underline{w}$ verifies the special triangle equality

$$
\underline{w}\left(\Phi_{1}\right) \neq \underline{w}\left(\Phi_{2}\right) \Rightarrow \underline{w}\left(\Phi_{1}+\Phi_{2}\right)=\operatorname{Min}\left(\underline{w}\left(\Phi_{1}\right), \underline{w}\left(\Phi_{2}\right)\right) .
$$


Fact 4.1.10. Let $V$ be any $n$-dimensional non-degenerate 0 -elliptic space and let $\left(f_{i}\right)$ be any fixed orthogonal basis of $V$, which is bounded by some $\varepsilon^{\prime}$ in $G$. Given $\phi \in L$, let

$$
\underline{w}(\phi)=\underline{w}(\operatorname{Mtrx}(\phi)) \text {. }
$$

Then:

(1) If $\phi \equiv \phi^{\prime}$, then $\left|\underline{w}(\phi)-\underline{w}\left(\phi^{\prime}\right)\right|<2 \varepsilon^{\prime}$.

(2) If $\sigma, \sigma^{\prime} \in U_{w(2)}^{+}$and if $\phi, \phi^{\prime}=\mathscr{C}(\sigma), \mathscr{C}\left(\sigma^{\prime}\right)$ are such that

$$
2 \underline{w}\left(\phi, \phi^{\prime}\right)>\underline{w}\left(\left(\phi, \phi^{\prime}\right), \phi^{\prime}\right)+5 \varepsilon^{\prime}
$$

then if $\gamma=\left[\left[\sigma, \sigma^{\prime}\right], \sigma^{\prime}\right]$, then

$$
\left|\underline{w}(\gamma-1)-\underline{w}\left(\left(\phi, \phi^{\prime}\right), \phi^{\prime}\right)\right| \leq 4 \varepsilon^{\prime}
$$

Proof. 1. Since $V$ is 0 -elliptic it follows that if $\tau w \geq g$ then $\operatorname{Mtrx}(\tau) w$ $\geq g-\varepsilon$ and that if $\operatorname{Mtrx}(\tau) w \geq g$ then $\tau w \geq g-\varepsilon(\S 3$, Theorem 3.5). In terms of the vector valuation $\underline{w}(\tau)$ this means that

$$
\begin{aligned}
& \tau w \geq g \Rightarrow \underline{w}(\tau) \geq g-\varepsilon^{\prime} \quad(g \in G) . \\
& \underline{w}(\tau) \geq g \Rightarrow \tau w \geq g-\varepsilon^{\prime} \quad(g \in G) .
\end{aligned}
$$

Let then $\phi \equiv \phi^{\prime}$. If $g=\underline{w}(\phi)$, then

$$
\underline{w}(\phi)=g \geq g \Rightarrow \phi w \geq g-\varepsilon^{\prime} .
$$

Since $\phi^{\prime} \equiv \phi$ it follows that $\phi^{\prime} w \geq g-\varepsilon^{\prime}$ so that $\underline{w}\left(\phi^{\prime}\right) \geq g-2 \varepsilon^{\prime}$ or, $\underline{w}\left(\phi^{\prime}\right)+2 \varepsilon^{\prime} \geq \underline{w}(\phi)$. By symmetry, $\underline{w}(\phi)+2 \varepsilon^{\prime} \geq \underline{w}\left(\phi^{\prime}\right)$. Thus $\mid w(\phi)-$ $w\left(\phi^{\prime}\right) \mid \leq 2 \varepsilon^{\prime}$, as desired.

(2) From the preceding fact follows

$$
\gamma-1 \equiv\left(\tau_{1}+\tau_{2}\right)+\tau_{3}
$$

where

$$
\begin{gathered}
\tau_{1}=\mu_{1}\left(\phi, \phi^{\prime}\right) \mu_{2}\left(\phi, \psi^{\prime}\right) \mu_{3}, \tau_{2}=\mu_{4}\left(\phi, \phi^{\prime}\right) \mu_{5}\left(\phi, \phi^{\prime}\right) \mu_{6}, \\
\tau_{3}=\mu_{7}\left(\left(\phi, \phi^{\prime}\right), \phi^{\prime}\right) \mu_{8}
\end{gathered}
$$

with all the $\mu_{i}$ in $M$. Thus from (1) follows

$$
\left|\underline{w}(\gamma-1)-\underline{w}\left(\left(\tau_{1}+\tau_{2}\right)+\tau_{3}\right)\right| \leq 2 \varepsilon^{\prime} .
$$

Now,

$$
\tau_{1} \equiv\left(\phi, \psi^{\prime}\right) \mu_{2}\left(\phi, \phi^{\prime}\right)
$$

Thus from, again, (1)

$$
\underline{w}\left(\tau_{1}\right) \geq \underline{w}\left(\phi, \phi^{\prime}\right) \mu_{2}\left(\phi, \phi^{\prime}\right)-2 \varepsilon^{\prime} \geq 2 \underline{w}\left(\left(\phi, \phi^{\prime}\right)\right)+\underline{w}\left(\mu_{2}\right)-2 \varepsilon^{\prime},
$$


and, since, $\mu_{2}$ is medial and, hence, $\mu_{2} w \geq 0$ it follows that $\underline{w}\left(\mu_{2}\right) \geq-\varepsilon^{\prime}$ so that

$$
\begin{aligned}
\underline{w}\left(\tau_{1}\right) & \geq 2 \underline{w}\left(\left(\phi, \phi^{\prime}\right)\right)-3 \varepsilon^{\prime}>\underline{w}\left(\left(\phi, \phi^{\prime}\right), \phi^{\prime}\right)+5 \varepsilon^{\prime}-3 \varepsilon^{\prime} \\
& >\underline{w}\left(\left(\phi, \phi^{\prime}\right), \phi^{\prime}\right)+2 \varepsilon^{\prime} .
\end{aligned}
$$

By the same token,

$$
\underline{w}\left(\tau_{2}\right)>\underline{w}\left(\left(\phi, \phi^{\prime}\right), \phi^{\prime}\right)+2 \varepsilon^{\prime} .
$$

By the generalized triangle inequality follows

$$
\underline{w}\left(\tau_{1}+\tau_{2}\right)>\underline{w}\left(\left(\phi, \phi^{\prime}\right), \phi^{\prime}\right)+2 \varepsilon^{\prime} .
$$

Now, from

$$
\tau_{3}=\mu_{7}\left(\left(\phi, \phi^{\prime}\right), \phi^{\prime}\right) \mu_{8}
$$

follows

$$
\underline{w}\left(\tau_{3}\right) \leq \underline{w}\left(\left(\phi, \phi^{\prime}\right), \phi^{\prime}\right)+2 \varepsilon^{\prime}<\underline{w}\left(\tau_{1}+\tau_{2}\right) .
$$

By the special triangle equality follows

$$
\underline{w}\left(\left(\tau_{1}+\tau_{2}\right)+\tau_{3}\right)=\underline{w}\left(\tau_{3}\right) .
$$

In view of (14)

$$
\left|\underline{w}(\gamma-1)-\underline{w}\left(\tau_{3}\right)\right| \leq 2 \varepsilon^{\prime}
$$

follows. Thus

$$
\begin{aligned}
& \left|\underline{w}(\gamma-1)-\underline{w}\left(\left(\phi, \phi^{\prime}\right), \phi^{\prime}\right)\right| \\
& \quad \leq\left|\underline{w}(\gamma-1)-\underline{w}\left(\tau_{3}\right)\right|+\left|\underline{w}\left(\tau_{3}\right)-\underline{w}\left(\left(\phi, \phi^{\prime}\right), \phi^{\prime}\right)\right| \\
& \quad \leq 2 \varepsilon+2 \varepsilon=4 \varepsilon,
\end{aligned}
$$

as desired.

Fact 4.1.11. (a) If $\left(g_{1}, g_{2}\right)$ is in the equalizer of $P \pi_{0}$ then $\left(g_{1}, g_{2}\right)$ is in the equalizer of $\pi_{1}$.

(b) If $\left(g_{1}, g_{2}\right)$ is in the equalizer of $P \pi_{1}$ then:

(i) $\left[\pi_{1}\left(g_{1}, \pi_{0}\left(g_{1}\right)\right)\right] \subset \pi_{0}\left(2 g_{2}+g_{1}\right)$;

(ii) $\left[\pi_{1}\left(g_{2}\right), \pi_{0}\left(g_{2}\right)\right] \subset \pi_{0}\left(2 g_{1}+g_{2}\right)$.

Proof. (a) To say that $P \pi_{0}\left(g_{1}\right)=P \pi_{0}\left(g_{2}\right)$ is to say that

$$
\pi_{0}\left(g_{1}\right)(U \cap Z)=\pi_{0}\left(g_{1}\right)(U \cap Z) \text {. }
$$


Then

$$
\begin{aligned}
\pi_{1}\left(g_{1}\right) & =\left[\pi_{0}\left(g_{1}\right), \pi_{0}\left(g_{1}\right)\right]=\left[\pi_{0}\left(g_{1}\right)(U \cap Z), \pi_{0}\left(g_{1}\right)\right] \\
& =\left[\pi_{0}\left(g_{2}\right)(U \cap Z), \pi_{0}\left(g_{1}\right)\right]=\left[\pi_{0}\left(g_{2}\right), \pi_{0}\left(g_{1}\right)\right] \\
& =\left[\pi_{0}\left(g_{1}\right), \pi_{0}\left(g_{2}\right)\right]=\left[\pi_{0}\left(g_{2}\right), \pi_{0}\left(g_{2}\right)\right]=\pi_{1}\left(g_{2}\right) .
\end{aligned}
$$

(b) To say that $P \pi_{1}\left(g_{1}\right)=P \pi_{1}\left(g_{2}\right)$ is to say

$$
\pi_{1}\left(g_{1}\right)\left(U^{(1)} \cap Z\right)=\pi_{1}\left(g_{2}\right)\left(U^{(1)} \cap Z\right) .
$$

Then

$$
\begin{aligned}
& {\left[\pi_{1}\left(g_{1}\right), \pi_{0}\left(g_{1}\right)\right]=\left[\pi_{1}\left(g_{1}\right)\left(U^{(1)} \cap Z\right), \pi_{0}\left(g_{1}\right)\right]} \\
& \quad=\left[\pi_{1}\left(g_{2}\right)\left(U^{(2)} \cap Z\right), \pi_{0}\left(g_{1}\right)\right]=\left[\pi_{1}\left(g_{2}\right), \pi_{0}\left(g_{1}\right)\right] \\
& \quad \subset\left[\pi_{0}\left(2 g_{2}\right), \pi_{0}\left(g_{1}\right)\right] \subset \pi_{0}\left(2 g_{2}+g_{1}\right) .
\end{aligned}
$$

Similarly,

$$
\left[\pi_{1}\left(g_{2}\right), \pi_{0}\left(g_{2}\right)\right] \subset \pi_{0}\left(2 g_{1}+g_{2}\right) .
$$

Fact 4.1.12. Let $V$ be any non-degenerate 0 -elliptic space and let $V^{\prime}$ be any orthogonal summand of $V$. If $\mathscr{F}$ is one of the mappings $\pi_{0}=\pi_{0}(V)$, $P \pi_{0}=P \pi_{0}(V), P \pi_{1}=P \pi_{1}=(V), \pi_{1}, \ldots$, and if $\mathscr{F}^{\prime}$ is the corresponding mapping relative to the space $V^{\prime}$, then the equalizer of $\mathscr{F}$ is contained in that of $\mathscr{F}^{\prime}$.

Proof. $\left(\mathscr{F}=\pi_{0}, P \pi_{0}\right) \quad$ Assume that $\pi_{0}\left(g_{1}\right)=\pi_{0}\left(g_{2}\right)$. Since $V$ is 0 -elliptic it follows by $\S 2$, Theorem 2.6 that if $g \in G$, then

$$
L_{g}(V) \supset L_{g}\left(V^{\prime}\right) \oplus 1 \text {. }
$$

Thus

$$
U_{g}(V) \supset U_{g}\left(V^{\prime}\right)+1
$$

Then

$$
U_{g}\left(V^{\prime}\right) \oplus 1=\left(U\left(V^{\prime}\right) \oplus 1\right) \cap U_{g}(V) .
$$

From $\pi_{0}\left(g_{1}\right)=\pi_{0}\left(g_{2}\right)$ follows

$$
\begin{aligned}
\pi_{0}^{\prime}\left(g_{1}\right) \oplus 1 & =U_{g_{1}}\left(V^{\prime}\right) \oplus 1=\left(U\left(V^{\prime}\right) \oplus 1\right) \cap U_{g_{1}}(V) \\
& =\left(U\left(V^{\prime}\right)+1\right) \cap U_{g_{2}}(V)=\pi_{0}^{\prime}\left(g_{2}\right) \oplus 1
\end{aligned}
$$

and, consequently, $\pi_{0}^{\prime}\left(g_{1}\right)=\pi_{0}^{\prime}\left(g_{2}\right)$.

Assume that $V^{\prime} \neq V$ and that $P \pi_{0}\left(g_{1}\right)=P \pi_{0}\left(g_{2}\right)$. This means that

$$
U_{g_{1}} \cdot(Z \cdot 1 \cap U)=U_{g_{2}} \cdot(Z \cdot 1 \cap U)
$$


where $1=$ identity mapping of $V$. Denote by $1^{\prime}$ the identity mapping of $V^{\prime}$. It is to be shown that

$$
U_{g_{1}}\left(V^{\prime}\right) \cdot\left(Z \cdot 1^{\prime} \cap U\left(V^{\prime}\right)\right)=U_{g_{2}}\left(V^{\prime}\right) \cdot\left(Z \cdot 1 \cap U\left(V^{\prime}\right)\right) .
$$

Let $\sigma^{\prime} \in U_{g_{1}}\left(V^{\prime}\right)$ and let $z \in Z$ be such that $z \cdot 1^{\prime} \in U\left(V^{\prime}\right)$. Equivalently, $z$ is a central unitary in the ground division ring $(D ; *)$. If $\sigma_{1}=\sigma^{\prime} \oplus 1^{\prime \prime}$, where $1^{\prime \prime}=$ identity mapping of $V^{\prime \perp}$, then $\sigma_{1} \in U_{g_{1}}\left(V^{\prime}\right) \oplus 1^{\prime \prime} \subset U_{g_{1}}(V)$. Thus $\sigma_{1}(z \cdot 1) \in U_{g_{1}}(V) \cdot(Z \cdot 1 \cap U)=U_{g_{2}}(V) \cdot(Z \cdot \cap U)$. This means that for some $\sigma_{2} \in U_{g_{2}}(V)$ and some $z_{2}$, a central unitary element in $(D ; *)$,

$$
\sigma_{1}(z \cdot 1)=\sigma_{2}\left(z_{2} \cdot 1\right) .
$$

From the preceding equation follows

$$
V^{\prime} \sigma_{2}=z_{2} V^{\prime} \sigma_{2}=V^{\prime} \sigma_{2}\left(z_{2} \cdot 1\right)=V^{\prime} \sigma_{1}\left(z_{1} \cdot 1\right)=z_{1} V^{\prime} \sigma_{1}=V^{\prime}
$$

and

$$
V^{\prime \perp} \sigma_{2}=V^{\prime \perp} \sigma_{2}\left(z_{2} \cdot 1\right)=V^{\prime \perp} \sigma_{1}(z \cdot 1)=V^{\prime \perp} .
$$

Thus $\sigma_{2}=\sigma_{2}^{\prime} \oplus \sigma_{2}^{\prime \prime}$, where $\sigma_{2}^{\prime}=\sigma_{2} / V^{\prime}$ and $\sigma_{2}^{\prime \prime}=\sigma_{2} / V^{\prime \prime}$. Moreover, if $V^{\prime \prime} \in V^{\prime \perp}$, then

$$
\begin{aligned}
v^{\prime \prime} \sigma_{2}^{\prime \prime} & =v^{\prime \prime} \sigma_{2}=z_{2}^{-1} v^{\prime \prime} z_{2} \sigma_{2} \\
& =z_{2}^{-1} v^{\prime \prime} z \sigma_{1}=z_{2}^{-1} z v^{\prime \prime} \sigma_{1}=z_{2}^{-1} z v^{\prime \prime} .
\end{aligned}
$$

This means that $\sigma_{2}^{\prime \prime}=z_{2}^{-1} z \cdot 1^{\prime \prime}$, where $1^{\prime \prime}=$ identity mapping of $V^{\prime \perp}$. Therefore,

$$
\begin{aligned}
z \sigma_{1} & =z\left(\sigma^{\prime} \oplus 1^{\prime \prime}\right)=z=\left(\sigma^{\prime} \oplus 1^{\prime \prime}\right)(z \cdot 1) \\
& =\sigma_{2}\left(z_{2} \cdot 1\right)=z_{2}\left(\sigma_{2}^{\prime} \oplus z_{2}^{-1} z 1^{\prime \prime}\right) .
\end{aligned}
$$

Then $z \sigma_{1}^{\prime}=z_{2} \sigma_{2}^{\prime}$, and, hence

$$
\sigma_{1}^{\prime} \cdot\left(z \cdot 1^{\prime}\right)=\sigma_{2}^{\prime}\left(z_{2} \cdot 1^{\prime}\right),
$$

where $\sigma_{2}^{\prime} \in U_{g_{2}}\left(V^{\prime}\right)$ for $\sigma_{2} \in U_{g_{2}}(V)$ and $\sigma_{2}^{\prime}=\sigma_{2} / V^{\prime}$, and $z_{2}$ is a central unitary. Thus

$$
\sigma_{1}^{\prime \cdot}\left(z \cdot 1^{\prime}\right) \in U_{g_{2}}\left(V^{\prime}\right)^{j} \cdot\left(Z \cap U\left(V^{\prime}\right)\right) .
$$

As this holds for each pair $\sigma_{1}^{\prime} \in U_{g_{1}}\left(V^{\prime}\right)$ and $z \in \mathscr{U}(D ; *) \cap Z$ it follows that

$$
U_{g_{1}}\left(V^{\prime}\right) \cdot\left(Z \cap U\left(V^{\prime}\right)\right) \subset U_{g_{2}}\left(V^{\prime}\right) \cdot\left(Z \cap U\left(V^{\prime}\right)\right) .
$$

By symmetry,

$$
U_{g_{2}}\left(V^{\prime}\right) \cdot\left(Z \cap U\left(V^{\prime}\right)\right) \subset U_{g_{1}}\left(V^{\prime}\right) \cdot\left(Z \cap U\left(V^{\prime}\right)\right) .
$$


Therefore

$$
p \pi_{0}^{\prime}\left(g_{1}\right)=P \pi_{0}^{\prime}\left(g_{2}\right)
$$

as desired.

4.2. The mapping $g \rightarrow P U_{g}^{(1)}$ for $\operatorname{dim}_{D} V>1$.

Generally the mapping $P \pi_{1}=g \in(w(2), \rightarrow)=P U_{g}^{(1)} \in \operatorname{lat}\left(P U^{(1)}\right)$ has a non-trivial equalizer even when $\operatorname{dim}_{D} V>1$. Here is an example where $P \pi_{1}$ is constant.

REMARK 4.2.1. If $V$ is the 2-dimensional orthogonal space then $P \pi_{1}$ is the constant mapping. In fact, $\pi_{1}$ is the constant mapping.

Proof. Pick any orthogonal basis $f_{1}, f_{2}$ of $V$. If $s_{i}=f_{\imath} \cdot f_{l}, i=1,2$, and $\lambda=s_{1} s_{2}^{-1}$ then by inspection, $\phi \in U \Leftrightarrow \underline{\phi}=\operatorname{Mtrx}(\phi)$ is of one of the following types.

$$
\begin{array}{cl}
\underline{\phi}\left|\begin{array}{ll}
a & b \\
-\lambda b & a
\end{array}\right| & (\operatorname{det} \underline{\phi}=1) ; \\
\underline{\phi}=\left|\begin{array}{ll}
a & b \\
\lambda b & a
\end{array}\right| & (\operatorname{det} \underline{\phi}=-1) .
\end{array}
$$

Thus $1+\phi$ is invertible if and only if $\operatorname{det} \phi=1$. Since the set of matrices $\phi$ as in (17) form an abelian normal subgroup of invertible matrices and since $1+\phi$ is invertible for every $\phi \in U_{g}$ where $g>w(2)$, it follows that $U_{g}$ is abelian so that $\pi_{1}(g)=1$ for every $g>w(2)$.

THEOREM 4.2.2. Let $V$ be any non-degenerate 0-elliptic space with dimension $>1$. Assume that $V$ is of one of the following types.

(1) $V$ is a non-orthogonal space containing some non-zero orthogonal vectors $f_{1}$ and $f_{2}$ such that

$$
\left|w\left(f_{1} \cdot f_{1}\right)-w\left(f_{2} \cdot f_{2}\right)\right| \leq n \varepsilon
$$

where $n$ is any fixed natural number and $\varepsilon$ is either the zero of or else the first positive element in $G$.

(2) $V$ is an orthogonal space containing a triple of non-zero orthogonal vectors $f_{1}, f_{2}, f_{3}$ such that

$$
\left|w\left(f_{i} \cdot f_{i}\right)-w\left(f_{j} \cdot f_{J}\right)\right| \leq n \varepsilon \quad(i, j=1,2,3),
$$

where $n$ and $\varepsilon$ are as in 1 .

Then:

(3) There is an isomorphism $\gamma$ of the chain $G^{+}$into $G^{+}$such that $\gamma \circ P \pi_{2}$ $\left(=g \in G^{+} \rightarrow\left[U_{\gamma(g)}, U_{\gamma(g)}\right] /[U, U] \cap Z\right)$ is a dual isomorphism from $G^{+}$ into lat. $(P[U, U])$, the lattice of normal subgroups of the projective special unitary group. 
Proof. Case where $V$ is as in 1. To say that $V$ is not orthogonal is evidently to say that the ground involution $*$ is not the identity mapping. Thus if $0 \neq s=s^{*} \in D$ then the co-gredient involution sending $x \in D$ onto $x^{(s)}=s x^{*} s^{-1}$ is, again, not the identity mapping. Without loss of generality one may assume that if $s_{i}=f_{i} \cdot f_{i}, i=1,2$, then $w\left(s_{1}\right) \leq w\left(s_{2}\right)$. Since $0 \neq s_{1}=s_{1}^{*}$ it follows that $\left(s_{1}\right)$ is not the identity mapping. If $0 \neq k \in D$ is such that $k \in \mathscr{K}\left(D ;\left(s_{1}\right)\right)$ then so are $2 k$ and $k^{-1}$ in $\mathscr{K}\left(D ;\left(s_{1}\right)\right)$. Hence one can find $k_{1} \in \mathscr{K}\left(D ;\left(s_{1}\right)\right)$ such that if $g_{0}=w\left(k_{1}\right)$ then $\infty \neq g_{0} \geq w(2)$. Let $\gamma: G^{+} \rightarrow G^{+}$be defined by

$$
\gamma(g)=g_{0}+2 m g
$$

where $m \geq 1$ is a natural number to be fixed later. If $g \in G$ and $x^{\prime} \in D$ is such that $w\left(x^{\prime}\right)=g$ then

$$
\begin{aligned}
g_{0}+2 g & =w\left(k_{1}\right)+2 w\left(x^{\prime}\right) \\
& =w\left(k_{1}\right)+w\left(x^{\prime} x^{\prime\left(s_{1}\right)}\right)=w\left(x^{\prime} k_{1} x^{\prime\left(s_{1}\right)}\right),
\end{aligned}
$$

where $x^{\prime} k_{1} x^{\prime\left(s_{1}\right)} \cap \mathscr{K}\left(D ;\left(s_{1}\right)\right)$. Thus

$$
g_{0}+2 g \in w\left(\mathscr{K}\left(D ;\left(s_{1}\right)\right)\right) .
$$

Therefore

$$
\gamma\left(G^{+}\right) \subset w\left(\mathscr{K}\left(D ;\left(s_{1}\right)\right)\right)
$$

and by construction,

$$
\gamma\left(G^{+}\right) \subset(w(2), \rightarrow) .
$$

Thus $\gamma \circ P \pi_{1}$ is a well-defined dual homomorphism from $G^{+}$into lat. $(P[U, U])$. It remains to show that $\gamma \circ P \pi_{1}$ is one-to-one. In view of Fact 4.1 .12 one may assume that $V$ is the subspace that is spanned by $f_{1}$ and $f_{2}$. Hence $\left(f_{1}, f_{2}\right)$ is an orthogonal basis of $V$, which is bounded by $\varepsilon^{\prime}=n \varepsilon$.

Let then $g_{1}, g_{2} \in \gamma\left(G^{+}\right)$be such that $P \pi_{1}\left(g_{1}\right)=P \pi_{1}\left(g_{2}\right)$. It is to be shown that for $m$ large enough ( $m$ depending only on $(n, \varepsilon)) g_{1}=g_{2}$ follows. Pick $x \in D$ such that $w(x)=g_{1}+n \varepsilon=g_{1}+\varepsilon^{\prime}$ and $\mathscr{K}=-k^{(s)}$ such that $w(k)=g_{1}+2 \varepsilon^{\prime}$ (possible since

$\left.g_{1}+2 \varepsilon^{\prime}=g_{1}+2 n \varepsilon \in \gamma\left(G^{+}\right)+2 G \geq 0 \subset g_{0}+2 G \subset w\left(\mathscr{K}\left(D ;\left(S_{1}\right)\right)\right)\right)$.

Define $\phi, \phi^{\prime} \in L(V)$ by the matrix equations

$$
\begin{gathered}
\operatorname{Mtrx}(\phi)=\left|\begin{array}{cc}
0 & x \\
-s_{2} x^{\prime} s_{1}^{-1} & 0
\end{array}\right| \\
\operatorname{Mtrx}\left(\phi^{\prime}\right)=\left|\begin{array}{ll}
k & 0 \\
0 & 0
\end{array}\right| .
\end{gathered}
$$


By construction, $\phi$ and $\phi^{\prime}$ are skew-symmetric transformations and by inspection,

$$
\begin{gathered}
\underline{w}(\phi) \quad(=\underline{w}(\operatorname{Mtrx}(\phi)))=g_{1}+\varepsilon^{\prime} \\
\underline{w}\left(\phi^{\prime}\right)=g_{1}+2 \varepsilon^{\prime} .
\end{gathered}
$$

From the preceding relations follows that

$$
\phi, \phi^{\prime} \in \mathscr{K}_{g_{1}}(V) \text {; }
$$

in fact, $\phi w \geq\left(g_{1}+\varepsilon^{\prime}\right)-\varepsilon^{\prime}$ and $\phi^{\prime} w \geq\left(g_{1}+2 \varepsilon^{\prime}\right)-\varepsilon^{\prime}=g_{1}+\varepsilon^{\prime} \geq g_{1}$. Since $g_{1}>w(2)$ it follows that if $\sigma, \sigma^{\prime}=\mathscr{C}^{-1}(\phi), \mathscr{C}^{-1}\left(\phi^{\prime}\right)$ then $\sigma, \sigma^{\prime}$ are well-defined unitary transformations of $V$ and that $\sigma, \sigma^{\prime} \in U_{g_{1}}(V)$.

Now, by inspection

$$
\begin{aligned}
\underline{w}\left(\left(\phi, \phi^{\prime}\right)\right) & =\underline{w}(\phi)+\underline{w}\left(\phi^{\prime}\right)=2 g_{1}+3 \varepsilon^{\prime}, \\
\underline{w}\left(\left(\phi, \phi^{\prime}\right), \phi^{\prime}\right) & =\underline{w}\left(\phi, \phi^{\prime}\right)+\underline{w}\left(\phi^{\prime}\right)=3 g_{1}+5 \varepsilon^{\prime} .
\end{aligned}
$$

To ensure the inequality

$$
2 \underline{w}\left(\left(\phi, \phi^{\prime}\right)\right)>\underline{w}\left(\left(\phi, \phi^{\prime}\right), \phi^{\prime}\right)+5 \varepsilon^{\prime}
$$

it suffices then to ensure that

$$
4 g_{1}+6 \varepsilon^{\prime}>3 g_{1}+10 \varepsilon^{\prime}
$$

or,

$$
g_{1}>4 \varepsilon^{\prime}=4 n \varepsilon .
$$

If $\varepsilon=0$, then $g_{1}=g_{0}+2 m g>0$ will follow for $g>0$ and $g_{0} \geq w(2)$ $\geq 0$.

If $\varepsilon$ is the first positive element in $G$ then $g_{1}=g_{0}+2 m g>4 n \varepsilon$ as soon as

$$
g_{1}+5 \varepsilon^{\prime} \geq g_{2}
$$

By symmetry, $g_{2}+5 \varepsilon^{\prime} \geq g_{1}$. Thus

$$
\left|g_{1}-g_{2}\right| \leq 5 \varepsilon^{\prime}
$$

If $\varepsilon=0$ then $\varepsilon^{\prime}=n \varepsilon=0$ giving $g_{1}=g_{2}$. If $\varepsilon$ is the first positive element in $G$ then since $g_{1}, g_{2} \in \gamma\left(G^{+}\right)=g_{0}+2 m G^{+}$it follows that $g_{1}-g_{2} \neq 0$ implies $\left|g_{1}-g_{2}\right| \geq 2 m \varepsilon \geq 6 n \varepsilon=6 \varepsilon^{\prime}$, contradicting the preceding inequality. This shows that $g_{1}=g_{2}$, as desired. Therefore $\gamma \circ P \pi_{1}$ is one-to-one as soon as $\gamma=\gamma_{m}=g \rightarrow g_{0}+2 m g$ is such that $m \geq 3 n$.

Case $V$ is an orthogonal space as in 2.

Take $\gamma=\gamma_{n}=g \mapsto w(2)+2 n g\left(g \in G^{+}\right)$. This is an isomorphism of $G^{+}$into $G^{+}$. It is to be shown that $\gamma \circ P \pi_{1}$ is one-to-one. 
Now, from $g>w(2)$ follows $-1 \notin U_{g}$ and, consequently, $-1 \notin$ $\pi_{1}(g) \subset U_{g}$. Thus $\pi_{1}(g) \cap([U, U] \cap Z) \supset \pi_{1}(g) \cap\{1,-1\}=(1)$. From this $\pi_{1}$ and $P \pi_{1}$ have same equalizers. Thus $\gamma \circ \pi_{1}$ and $\gamma \circ P \pi_{1}$ have same equalizers. It remains then to show that $\gamma \circ \pi_{1}$ has trivial equalizer. In view of Fact 4.1.12, one may assume that $V$ is spanned by the vectors $f_{l}$ as in the statement of 2 . Without loss of generality if $s_{i}=f_{\imath} \cdot f_{i}$, then $w\left(s_{1}\right) \leq$ $w\left(s_{2}\right) \leq w\left(s_{3}\right)$.

Pick any $x \in D$ such that $w(x)=g_{1}+\varepsilon^{\prime}\left(\varepsilon^{\prime}=n \varepsilon\right)$. Determine $\phi, \phi^{\prime}$ $\in L(V)$ by their matrix equations

$$
\begin{aligned}
\operatorname{Mtrx}(\phi) & =\left|\begin{array}{cr:c}
0 & x & 0 \\
-s_{2} s_{1}^{-1} s & 0 & 0 \\
\hdashline 0 & 0 & 0
\end{array}\right|, \\
\hdashline \operatorname{Mtrx}\left(\phi^{\prime}\right) & =\left|\begin{array}{cc:c}
0 & 0 & x \\
0 & 0 & 0 \\
\hdashline-s_{3} s_{1}^{-1} z & 0 & 0
\end{array}\right| .
\end{aligned}
$$

By inspection, $\phi, \phi^{\prime}$ are skew-symmetric transformations of $V$ such that

$$
\begin{aligned}
& \underline{w}(\phi)=g_{1}+\varepsilon^{\prime}, \\
& \underline{w}\left(\phi^{\prime}\right)=g_{1}+\varepsilon^{\prime},
\end{aligned}
$$

and

$$
\begin{aligned}
\underline{w}\left(\left(\phi, \phi^{\prime}\right)\right) & =\underline{w}\left|\begin{array}{lc:c}
0 & 0 & 0 \\
0 & 0 & -s_{2} s_{1}^{-1} x^{2} \\
\hdashline 0 & s_{3} s_{1}^{-1} x^{2} & 0
\end{array}\right| \\
& =\underline{w}(\phi)+\underline{w}\left(\phi^{\prime}\right)+w\left(s_{2} s_{1}^{-1}\right) .
\end{aligned}
$$

Since the basis $\left(f_{1}, f_{2}, f_{3}\right)$ is bounded by $\varepsilon^{\prime}=n \varepsilon$ it follows that $\phi, \phi^{\prime} \in$ $\mathscr{K}_{g_{1}}(V)$. In view of Fact $4.1 .8,\left(\phi, \phi^{\prime}\right) w \geq 2 g_{2}$ follows, and, consequently,

$$
\underline{w}\left(\left(\phi, \phi^{\prime}\right)\right) \geq 2 g_{2}-\varepsilon^{\prime} \text {. }
$$

Thus

$$
2 g_{1}+w\left(s_{2} s_{1}^{-1}\right)=\underline{w}\left(\left(\phi, \phi^{\prime}\right)\right) \geq 2 g_{2}-\varepsilon^{\prime}
$$

or,

$$
2 g_{1}+2 \varepsilon^{\prime} \geq 2 g_{1}+w\left(s_{2} s_{1}^{-1}\right)+\varepsilon^{\prime} \geq 2 g_{2} ; g_{1}+\varepsilon^{\prime} \geq g_{2} .
$$

By symmetry, $g_{2}+\varepsilon^{\prime} \geq g_{1}$. Thus $\left|g_{1}-g_{2}\right| \leq \varepsilon^{\prime}$. If $\varepsilon=0$ then $\varepsilon^{\prime}=n \varepsilon=0$ follows giving $g_{1}=g_{2}$. If $\varepsilon$ is the first positive element in $G$ then $g_{1} \neq g_{2}$ 
implies $\left|g_{1}-g_{2}\right| \geq 2 n \varepsilon=2 \varepsilon^{\prime}>\varepsilon^{\prime}$, contradicting the inequality $\left|g_{1}-g_{2}\right|$ $\leq \varepsilon^{\prime}$. This shows that $\gamma_{n} \circ P \pi_{1}$ is one-to-one, which concludes the proof.

Corollary 4.2.3. If $V$ is as in Theorem 4.2.2 then the positive cone $G^{+}$ of the underlying value group $G$ can be dually embedded in lat. $\left(P U^{(1)}\right)$, the lattice of normal subgroups of the projective derived unitary group $P U^{(1)}=$ $[U, U] /[U, U] \cap Z$.

Proof. For $\gamma \circ P \pi_{1}$ is a dual homomorphism of ordered sets.

COROllary 4.2.4. If $V$ is as in Theorem 4.2.2 then $\gamma \circ P \pi_{0}$ is a dual one-to-one homomorphism from $G^{+}$into lat. $\left(P U^{(0)}\right)$, the lattice of normal subgroups of the projective unitary group $P U^{(0)}=U / U \cap Z$.

Proof. For the equalizer of $\gamma \circ P \pi_{0}$ is contained in the equalizer of $\gamma \circ \pi_{1}$. The latter is contained in the equalizer of $\gamma \circ P \pi_{1}$.

\subsection{The mapping $g \rightarrow P U_{g}^{(1)}$ for $\operatorname{dim}_{D} V=1$.}

Will the restriction $\operatorname{dim}_{D} V=1$ make the treatment of the mapping $g \rightarrow\left[U_{g}, U_{g}\right] /[U, U] \cap Z$ easier? As it turns out it is the reverse that happens to be true. I suggest as an explanation the following. Theorem 4.2.2 was established by making explicit use of skew-symmetric transformations $\phi$ and $\phi^{\prime}$ with prescribed values relative to the vector valuation $\underline{w}$ and such that

$$
\underline{w}\left(\phi \phi^{\prime}-\phi^{\prime} \phi\right)=\underline{w}(\phi)+\underline{w}\left(\phi^{\prime}\right) .
$$

(See equations (21)-(26), (29)). In dealing with the simplest instance of 1-dimensional space $V$, namely the space ${ }_{D} D$, relative to the form $x \cdot y=$ $x y^{*},(28)$ means that for certain skew-symmetrics $\phi, \phi^{\prime}$ in $(D ; *)$ with prescribed values $w(\phi)=g, w\left(\phi^{\prime}\right)=g^{\prime}$,

$$
w\left(\phi \phi^{\prime}-\phi^{\prime} \phi\right)=w(\phi)+w\left(\phi^{\prime}\right) .
$$

I do not know of any standard construction of such pairs $\phi, \phi^{\prime}$ for the given $*$-valued division ring $D$ As a matter of fact there is a clear-cut class of division rings $D$ possibly infinite dimensional, in which, equality (29) never occurs even if $\phi, \phi^{\prime}$ are any elements in $D$ (see [10] or [4]).

Such division rings $D$ have commutative residue division rings $\bar{D}$. Reversing the trend, I will assume in what will follow that $\operatorname{dim}_{\bar{Z}} \bar{D}>4$, where $\bar{Z}=\operatorname{center}(\bar{D})$ and $1 / 2$ exists in $\bar{D}$. 
Theorem 4.3.1. Let $(D ; * ; w)$ be any $*$-valued division ring. Assume that $1 / 2$ exists in the valuation ring $R$ and that the residue division ring $D$ is of dimension over its center exceeding 4. For each pair of skew-symmetrics $\sigma$ and $\tau$ one can find symmetric units $u$ and $v$ in $R$ such that if $\sigma^{\prime}=u \sigma u$ and $\tau^{\prime}=v \tau v$ then

$$
w\left(\sigma^{\prime} \tau-\tau^{\prime} \sigma^{\prime}\right)=w\left(\sigma^{\prime}\right)+w\left(\tau^{\prime}\right) .
$$

Proof. Deny the conclusion of the theorem. In particular,

$$
w(\sigma \tau-\tau \sigma) \neq w(\sigma)+w(\tau) .
$$

Thus $\sigma \neq 0$ and $\tau \neq 0$. Then

$$
\begin{aligned}
w(\sigma \tau-\tau \sigma) & =w(\sigma \tau(1-[\tau, \sigma])) \\
& =w(\sigma)+w(\tau)+w(1-[\tau, \sigma]) \neq w(\sigma)+w(\tau)
\end{aligned}
$$

so that $w(1-[\tau, \sigma]) \neq 0$. Since $[\tau, \sigma]$ is in $R$, it follows that $w(1-[\tau, \sigma])$ $>0$ and, hence,

$$
[\tau, \sigma] \equiv 1 \quad(\bmod J)
$$

I proceed to show that

$$
\left(u u^{\sigma}\right)^{\tau} \equiv u u^{\sigma} \quad(\bmod J),
$$

where $u$ is any symmetric unit in $R$, and $a^{b}=b^{-1} a b(a, b \in D)$. By standard properties of group commutators, if $u$ is any symmetric unit in $R$, then

$$
[u \tau u, \sigma]=[u, \sigma]^{u}[\tau u, \sigma]=[u, \sigma]^{\tau u}[\tau, \sigma]^{u}[u, \sigma] .
$$

Since $(\cdot)^{u}: x \in R \rightarrow x^{u}$ is an inner automorphism of the ring $R$ preserving evidently $J$ it follows that

$$
[\tau, \sigma]^{u} \equiv 1^{u}=1 \quad(\bmod J),
$$

giving

$$
[u \tau u, \sigma] \equiv[u, \sigma]^{\tau u}[u, \sigma] .
$$

By (30) applied to the pair $u \tau u$ and $\sigma$ it follows that

$$
[u, \sigma]^{\tau u}[u, \sigma] \equiv 1 \quad(\bmod J) .
$$

Conjugating by $u^{-1}$ the preceding congruence:

$$
[u, \sigma]^{\tau}[u, \sigma]^{u^{-1}} \equiv 1 \quad(\bmod J) .
$$

Equivalently,

$$
[u, \sigma]^{\tau} \equiv[\sigma, u]^{u^{-1}}
$$


Now,

$$
\begin{aligned}
{[\sigma, u]^{u^{-1}} } & =u[\sigma, u] u^{-1}=u \sigma^{-1} u^{-1} \sigma u u^{-1} \\
& =u \sigma^{-1} u^{-1} \sigma=\left[u^{-1}, \sigma\right]
\end{aligned}
$$

Thus

$$
[u, \sigma]^{\tau} \equiv\left[u^{-1}, \sigma\right] \quad(\bmod J)
$$

Here

$$
\begin{aligned}
{[u, \sigma]^{\tau} } & =\left[u^{\tau}, \sigma^{\tau}\right]=\left[u^{\tau}, \sigma[\sigma, \tau]\right] \\
& =\left[u^{\tau},[\sigma, \tau]\right]\left[u^{\tau}, \sigma\right]^{[\sigma \tau]}=\left[u^{\tau}, 1\right]\left[u^{\tau}, \sigma\right]^{1}=\left[u^{\tau}, \sigma\right]
\end{aligned}
$$

and, consequently,

$$
\left[u^{\tau}, \sigma\right] \equiv\left[u^{-1}, \sigma\right] \quad(\bmod J)
$$

or,

$$
\left(u^{-1}\right)^{\tau}\left(\sigma^{-1} u^{\tau} \sigma\right) \equiv u\left(\sigma^{-1} u^{-1} \sigma\right) \quad(\bmod J) .
$$

Left multiply the preceding congruence by $u^{-1}$ and right multiply the resulting congruence by $\left(\sigma^{-1} u^{\tau} \sigma\right)^{-1}$. After replacing $u$ by $u^{-1}$ one gets precisely (31).

I proceed to show that the inner automorphisms $(\sigma)$ and $(\tau)$ induce equal automorphisms on the residue division ring $\bar{D}$. Congruence (31) is evidently true if $u$ is a symmetric in $J$. Thus (31) holds true for $u$ any symmetric in $R$ and, consequently, (31) holds true if $u$ is replaced by $1+u$. This substitution gives

$$
\begin{array}{rlr}
((1+u)(1+u \sigma))^{\tau} & =\left(1+u+u^{\sigma}+u u^{\sigma}\right)^{\tau} \\
& =1+\left(u+u^{\sigma}\right)^{\tau}+\left(u u^{\sigma}\right) \tau & (1+u)\left(1+u^{\sigma}\right) \\
& =1+\left(u+u^{\sigma}\right)+\left(u u^{\sigma}\right)
\end{array}
$$

so,

$$
u+u^{\sigma} \equiv\left(u+u^{\sigma}\right)^{\tau} .
$$

Reversing the roles of $\sigma$ and $\tau$ in (32):

$$
u+u^{\tau} \equiv\left(u+u^{\tau}\right)^{\sigma} .
$$

Now, from $[\sigma, \tau] \equiv 1$ follows $u^{\sigma \tau}=u^{\tau \sigma}$. Subtracting (33) from (32) and eliminating congruent terms, it follows that

$$
u^{\sigma}-u^{\tau} \equiv u^{\tau}-u^{\sigma}
$$


Since $1 / 2$ exists in $R$, it follows that $u^{\sigma}-u^{\tau} \equiv 0$ or $u^{\sigma} \equiv u^{\tau}$, for every $u=u^{*}$ in $R$. Since $\bar{D}$ is of dimension exceeding 4 over its own center it follows by a well-known theorem of Dieudonné that $\bar{D}$ is generated by its symmetrics. Thus $x^{\sigma} \equiv x^{\tau}$ for every $x \in R$.

I will show next that $(\cdot)^{\sigma}$ is of period 2 (or less) modulo $J$ and that

$$
u u^{\sigma} \equiv u^{\sigma} u \quad\left(u=u^{*} \in R\right) .
$$

Indeed, in (33) replacing $(\cdot)^{\tau}$ by $(\cdot)^{\sigma}$ this gives

$$
u+u^{\sigma} \equiv u^{\sigma}+u^{\sigma^{2}} ; \quad u \equiv u^{\sigma^{2}} .
$$

Same substitution in (31) gives

$$
u \cdot u^{\sigma} \equiv\left(u u^{\sigma}\right)^{\sigma}=u^{\sigma} u^{\sigma^{2}} \equiv u^{\sigma} \cdot u .
$$

I proceed to show that $(\cdot)^{\sigma}$ is, in fact, the trivial automorphism modulo $J$.

Replacing $u$ by $u+v$ in (34) where $u$ and $v$ are symmetrics in $R$ and eliminating congruent terms it follows that

$$
u v^{\sigma}+v u^{\sigma} \equiv u^{\sigma} v+v^{\sigma} u \text {. }
$$

Now,

$$
\left(v^{\sigma}\right)^{*}=\left(\sigma^{-1} v \sigma\right)^{*}=\sigma v^{*} \sigma^{-1} \equiv \sigma^{-1} v^{*} \sigma=\sigma^{-1} v \sigma=v^{\sigma} .
$$

Thus $v^{\sigma}$ is symmetric modulo $J$. Thus one can replace $v$ by $v^{\sigma}$ in (35). Then

$$
u v+v^{\sigma} u^{\sigma} \equiv u^{\sigma} v^{\sigma}+v u
$$

equivalently,

$$
u v-v u \equiv(u v-v u)^{\sigma}
$$

for every pair of symmetrics $u$ and $v$ in $R$. Since the residue division ring $\bar{D}$ is of dimension over its center exceeding 4 it follows by a well-known theorem of I. N. Herstein that $\bar{D}$ is generated by Lie commutators of symmetric elements in $\bar{D}$. From (36) follows that $(\cdot)^{\sigma}$ is the identity automorphism modulo $J$.

It is now easy to reach a contradiction. For let $u, v$ be symmetric units in $R$. By the preceding

$$
v^{\sigma} \equiv v
$$

If $\sigma$ is replaced by $u \sigma u$ this gives

$$
v \equiv v^{u \sigma u}=\left(v^{u}\right)^{\sigma u}=\left(\left(v^{u}\right)^{\sigma}\right)^{u}\left(v^{u}\right)^{u}=v^{u^{2}} .
$$

Thus $v u^{2} \equiv u^{2} v$, for every pair of symmetric units $u$ and $v$ in $R$. A final linearization on $u$ shows that the symmetrics in $\bar{D}$ commute contradicting thus the dimension of $\bar{D}$ (Dieudonné). 
Theorem 4.3.2. Let $(D ; * ; w)$ be as in Theorem 4.3.1. For each pair of non-zero skew symmetrics $\sigma$ and $\tau$ as in the conclusion of Theorem 4.3.1 there is some symmetric unit $u$ of the valuation ring $R$ such that if $\tau^{\prime}=u \sigma u$ or $\tau u$ and $\sigma^{\prime}=\sigma \tau-\tau \sigma$, then

$$
w\left(\sigma^{\prime} \tau^{\prime}-\tau^{\prime} \sigma^{\prime}\right)=w\left(\sigma^{\prime}\right)+w\left(\tau^{\prime}\right) .
$$

Proof. Deny the conclusion of the theorem. Repeating the argument in the proof of (30) for $\sigma$ replaced by $\sigma^{\prime}$ and $\tau$ replaced by $\tau^{\prime}$ it follows that

$$
\left[\sigma^{\prime}, \tau^{\prime}\right] \equiv 1 \quad(\bmod J)
$$

By construction, $\tau^{\prime}$ can be replaced by $\sigma$ as well and by $u \tau^{\prime} u$ for every symmetric unit $u$ in $R$. Repeating the argument in the proof of (31) for $\sigma$ replaced by $\sigma^{\prime}$ and $\tau$ by $u \tau^{\prime} u$ it follows that

$$
u u^{\sigma^{\prime}} \equiv\left(u u^{\sigma^{\prime}}\right)^{\tau^{\prime}} \quad\left(\tau^{\prime}=\sigma \text { or } \tau\right),
$$

for every symmetric $u$ in $R$. Denote by $c$ the element $u \cdot u^{\sigma^{\prime}}$ and by $\bar{c}$ the image of $c$ in $\bar{D}$. I proceed to show that $\bar{c} \notin \bar{Z}$, the center of $\bar{D}$, for some $u$.

For if $\bar{c}$ always belong to $\bar{Z}$ then replacing $u$ by $1+u$ and eliminating gives $u+u^{\sigma^{\prime}} \in \bar{Z}$. From the identity

$$
u^{2}-u\left(u+u^{\sigma^{\prime}}\right)+u u^{\sigma^{\prime}}=0
$$

follows that $\bar{u}$ is quadratic over $\bar{Z}$ for every symmetric $\bar{u}$ in $\bar{D}$. By standard argument $\bar{D}$ would be 4-dimensional (at most) over $\bar{Z}$, which is ruled out.

Pick any $c=u u^{\sigma^{\prime}}$ such that $\bar{c} \notin \bar{Z}$. For each symmetric unit $v$ in $R$, the inner automorphisms ( ) ${ }^{v \sigma v}$ and ( ) ${ }^{v \tau v}$ preserve $c$ modulo $J$; it suffices to choose $\tau^{\prime}=v \sigma v, v \tau v$ successively and to quote (37). It follows that $\left(v^{-1} \tau^{-1} v^{-1}\right),\left(v \sigma^{-1} v\right),\left(v^{-1} \tau v^{-1}\right),(v \sigma v)$ all preserve $c$ modulo $J$. Thus the product of these automorphisms preserve $c$ modulo $J$. Since

$$
\left(v^{-1} \tau^{-1} v^{-1}\right)\left(v \sigma^{-1} v\right)\left(v^{-1} \tau v^{-1}\right)(v \sigma v)=v^{-1}[\tau, \sigma] v
$$

it follows that $(\cdot)^{v^{-1}[\tau, \sigma] v}$ preserves $c$ modulo $J$.

I will show next that $[\tau, \sigma]$ maps onto a central element in $\bar{D}$. For $[\sigma, \sigma]$ is a unit in $R$. Thus if $\bar{\delta}$ is the image of $[\tau, \sigma]$ in $\bar{D}$ then the relation

$$
c^{v^{-1}[\tau, \sigma] v} \equiv c
$$

implies

$$
\left[\bar{c}, \bar{v}^{-1} \bar{\delta} \bar{v}\right]=1
$$

for every non-zero symmetric $\bar{v}$ in $\bar{D}$. Equivalently, $\bar{\delta}$ commutes with $\bar{c} \bar{v}$ for every non-zero symmetric $\bar{v}$ in $\bar{D}$. By I. N. Herstein's [10, Theorem 6.1.1] follows that $\bar{\delta} \in \bar{Z}$; in view of the fact that $\bar{c} \notin \bar{Z}$. 
Now $\bar{\delta}=[\tau, \sigma] \neq 1$, the unity of $\bar{D}$. For if $\bar{\delta}$ were the unity of $\bar{D}$, then $[\tau, \sigma] \equiv 1$, so that

$$
\begin{aligned}
w\left(\sigma^{\prime}\right) & =w(\sigma \tau-\tau \sigma)=w(\sigma \tau(1-[\tau, \sigma])) \\
& =w(\sigma \tau)+w(1-[\tau, \sigma])>w(\sigma)+w(\tau),
\end{aligned}
$$

contrary to the equality $w\left(\sigma^{\prime}\right)=w(\sigma)+w(\tau)$. Thus $1=[\tau, \sigma]$ is a unit in $R$ and evidently $1=[\tau, \sigma]$ maps onto a central element in $\bar{D}$. If $x \in R$, $x \notin J$ it is to be shown that

$$
x^{1-[\tau, \sigma]} \equiv x
$$

For

$$
\begin{aligned}
\overline{x^{1-[\tau, \sigma]}} & =\overline{(1-[\tau, \sigma])^{-1} \times(1-[\tau, \sigma])} \\
& ={\overline{(1-[\tau, \sigma])^{-1}} \bar{x}(1-[\tau, \sigma])}^{-1} \overline{(1-[\tau, \sigma])} .
\end{aligned}
$$

Since $1-[\tau, \sigma] \in \bar{Z}$ it follows that $(1-[\tau, \sigma])^{-1} \bar{x}(1-[\tau, \sigma])=\bar{x}$ giving

$$
x^{1-[\tau, \sigma]}=\bar{x}
$$

equivalently

$$
x^{1-[\tau, \sigma]} \equiv x .
$$

From the preceding congruence follows that for each symmetric unit $u$ in $R$

$$
u^{\sigma^{\prime}}=u^{\sigma \tau-\tau \sigma}=u^{\sigma \tau(1-[\tau, \sigma])}=\left(u^{\sigma \tau}\right)^{(1-[\tau, \sigma])} \equiv u^{\sigma \tau} .
$$

In view of (37),

$$
\begin{aligned}
u u^{\sigma \tau} & \equiv u u^{\sigma^{\prime}} \equiv\left(u u^{\sigma \tau-\tau \sigma}\right)^{\tau^{\prime}} \\
& =u^{\tau^{\prime}}\left(u^{\sigma \tau-\tau \sigma}\right)^{\tau^{\prime}} \quad u^{\tau}\left(u^{\sigma \tau}\right)^{\tau^{\prime}}=\left(u u^{\sigma \tau}\right)^{\tau^{\prime}}
\end{aligned}
$$

Since the preceding congruence holds for both cases $\tau^{\prime}=\sigma$ or $\tau^{\prime}=\tau$ it follows that if $\gamma=\sigma \tau$ then

$$
u u^{\gamma} \equiv\left(u u^{\gamma}\right)^{\gamma}
$$

In the preceding congruence replace $u$ by $1+u$ and eliminate congruent terms. This gives $u+u^{\gamma} \equiv\left(u+u^{\gamma}\right)^{\gamma}$ or, $u \equiv u^{\gamma^{2}}$. Returning to (40) and replacing $u^{\gamma^{2}}$ by $u$, one has $u u^{\gamma} \equiv u^{\gamma} u$. Exactly as in the proof of Theorem 4.3.1, argument following (36) one deduces from the preceding congruence that $\gamma$ induces the identity automorphism of $\bar{D}$. Thus from (37) follows

$$
u^{2} \equiv u u^{\sigma \tau-\tau \sigma} \equiv\left(u u^{\sigma \tau-\tau \sigma}\right)^{\tau^{\prime}} \equiv\left(u u^{\sigma \tau}\right)^{\tau^{\prime}} \equiv u^{2^{\tau^{\prime}}}
$$


By one more linearization argument follows that $u \equiv u^{\tau^{\prime}}$. Since $\tau^{\prime}$ can be replaced by $v \sigma v$ one deduces that the symmetrics in $\bar{D}$ commute, which is a contradiction.

THEOREM 4.3.3. Let $(D ; * ; w)$ be any $*$-valued division ring such that $1 / 2$ exists in the valuation ring $R$. Let $\phi_{1}, \phi_{2}, \phi_{3}$ be three non-zero skew-symmetrics such that

(i) $w\left(\phi_{1}\right)=w\left(\phi_{2}\right)=w\left(\phi_{3}\right)>0$, where if $g=w\left(\phi_{1}\right)$, then $g \neq \infty$;

(ii) $w\left(\phi_{1} \phi_{2}-\phi_{2} \phi_{1}\right)=2 g$;

(iii) $w\left(\phi_{1} \phi_{2}-\phi_{2} \phi_{1}\right) \phi_{3}-\phi_{3}\left(\phi_{1} \phi_{2}-\phi_{1} \phi_{1}\right)=3 g$.

Put $\sigma_{i}=\left(1-\phi_{i} / 2\right) /\left(1+\phi_{i} / 2\right)(i=1,2,3)$. Then the $\sigma_{i}$ are unitaries in $D$ such that

(iv) $w\left(\sigma_{i}\right)=g(i=1,2,3)$;

(v) $w\left(\left[\left[\sigma_{1}, \sigma_{2}\right], \sigma_{3}\right]-1\right)=3 g$.

Proof. (iv) At the risk of repeating slightly the calculations in Fact 4.1.7, point 1 , one has

$$
\sigma_{i}-\frac{1-\phi_{i} / 2}{1+\phi_{1} / 2}-1=\frac{-\phi_{i}}{1+\phi_{i} / 2}
$$

giving

$$
w\left(\sigma_{i}-1\right)=w\left(\phi_{i}\right)+w\left(1+\phi_{i} / 2\right)=w\left(\phi_{l}\right),
$$

for since $w\left(\phi_{i}\right)>0$ and $w(1 / 2)=0$ it follows that $w\left(1+\phi_{i} / 2\right)=0$.

(v) Represent $\left[\sigma_{1}, \sigma_{2}\right]$ in the form

$$
\left[\sigma_{1}, \sigma_{2}\right]=\frac{1-t / 2}{1+t / 2} \text {. }
$$

Indeed, the preceding equation can be solved for $t / 2$ provided $\left[\sigma_{1}, \sigma_{2}\right] \neq$ -1 . If $\left[\sigma_{1}, \sigma_{2}\right]$ were central then (iii) would be obviously violated. Thus $\left[\sigma_{1}, \sigma_{2}\right] \neq-1$. If $t_{1}=1-\left[\sigma_{1}, \sigma_{2}\right]$ then

$$
t / 2=\frac{t_{1}}{2-t_{1}}
$$

From trivial adaptations of Facts 4.1.7 and 4.1.9 follows that if $\gamma=$ $\left[\left[\sigma_{1}, \sigma_{2}\right], \sigma_{3}\right]$ then

$$
w(\gamma-1)=w\left(\left(\frac{1}{2-t_{1}}, \phi_{3}\right)\right)=w\left(\left(t_{1}, \phi_{3}\right)\right)=w\left(\phi_{3}, \sigma_{1}^{-1} \sigma_{2}^{-1}\left(\sigma_{1}, \sigma_{2}\right)\right) .
$$

Now,

$$
\begin{aligned}
\left(\phi_{3}, \sigma_{1}^{-1} \sigma_{2}^{-1}\left(\sigma_{1}, \sigma_{2}\right)\right)= & \left(\phi_{3}, \sigma_{1}^{-1}\right) \sigma_{2}^{-1}\left(\sigma_{1}, \sigma_{2}\right)+\sigma_{1}^{-1}\left(\phi_{3}, \dot{\varphi}_{2}^{-1}\right)\left(\sigma_{1}, \sigma_{2}\right) \\
& +\sigma_{1}^{-1} \sigma_{2}^{-1}\left(\phi_{3},\left(\sigma_{1}, \sigma_{2}\right)\right)=\tau_{1}+\tau_{2}+\tau_{3},
\end{aligned}
$$


where

$$
\begin{aligned}
& \tau_{1}=\left(\phi_{3}, \sigma_{1}^{-1}\right) \sigma_{2}^{-1}\left(\sigma_{1}, \sigma_{2}\right), \tau_{2}=\sigma_{1}^{-1}\left(\phi_{3}, \sigma_{2}^{-1}\right)\left(\sigma_{1}, \sigma_{2}\right), \\
& \tau_{3}=\sigma_{1}^{-1} \sigma_{2}^{-1}\left(\phi_{3},\left(\sigma_{1}, \sigma_{2}\right)\right) .
\end{aligned}
$$

Now

$$
\begin{aligned}
w\left(\tau_{1}\right) & =w\left(\phi_{3}, \sigma_{1}^{-1}\right)+w\left(\left(\sigma_{1}, \sigma_{2}\right)\right) \\
& =w\left(\left(\phi_{3}, \phi_{1}\right)\right)+w\left(\left(\phi_{1}, \phi_{2}\right)\right) \geq 4 g ;
\end{aligned}
$$

$w\left(\tau_{2}\right)>4 g$. Also,

$$
w\left(\tau_{3}\right)=w\left(\phi_{3},\left(\sigma_{1}, \sigma_{2}\right)\right)
$$

and

$$
\begin{aligned}
\left(\phi_{3},\left(\sigma_{1}, \sigma_{2}\right)\right) & =\left(\phi_{3}, \frac{1}{1-\phi_{1} / 2} \frac{1}{1-\phi_{2} / 2}\left(\phi_{2}, \phi_{1}\right) \frac{1}{1-\phi_{2} / 2} \frac{1}{1-\phi_{1} / 2}\right) \\
& =\tau_{1}^{\prime}+\tau_{2}^{\prime}+\tau_{3}^{\prime}+\tau_{4}^{\prime}+\tau_{5}^{\prime},
\end{aligned}
$$

where

$$
\tau_{3}^{\prime}=\frac{1}{1-\phi_{1} / 2} \frac{1}{1-\phi_{2} / 2}\left(\phi_{3},\left(\phi_{2}, \phi_{1}\right)\right) \frac{1}{1-\phi_{2} / 2} \frac{1}{1-\phi_{1} / 2}
$$

has value $w\left(\phi_{3},\left(\phi_{2}, \phi_{1}\right)\right)=3 g$ and all other terms $\tau_{i}^{\prime}, i \neq 3$, have values at least $4 \mathrm{~g}$. By the special triangle equality follows

$$
w\left(\phi_{3}, \sigma_{1}^{-1} \sigma_{2}^{-1}\left(\sigma_{1}, \sigma_{2}\right)\right)=w\left(\tau_{3}^{\prime}\right)=3 g,
$$

so

$$
w(\gamma-1)=3 g=w\left(\left(\phi_{1}, \phi_{2}\right), \phi_{3}\right)
$$

THEOREM 4.3.4. Let $(D ; * ; w)$ be any $*$-valued division ring such that $1 / 2$ exists in the valuation ring $R$ in $D$ and the residue division ring $\bar{D}$ has dimension over its own center exceeding 4 . Denote by $\mathscr{U}$ the unitary group of $D, P \mathscr{U}^{(1)}$ the factor group $[\mathscr{U}, \mathscr{U}] /[\mathscr{U}, \mathscr{U}] \cap Z$ and $P U_{g}^{(1)}$ the subgroup $\left[\mathscr{U}_{g}, \mathscr{U}_{g}\right] /[\mathscr{U}, \mathscr{U}] \cap Z$, where $\mathscr{U}_{g}=\{u \in \mathscr{U} \mid w(u-1) \geq g\}$. Then $g \rightarrow$ $P \mathscr{U}_{g}^{(1)}$ is a dual embedding of the positive cone $G^{+}$of $G$ into the lattice of normal subgroups of the group $P \mathscr{U}^{(1)}$.

Proof. By inspection, $\mathscr{U}_{g}$ is a normal subgroup of $\mathscr{U}$ which decreases as $g$ increases. Hence $g \rightarrow \stackrel{P}{P} \mathscr{U}_{g}$ is a dual homomorphism from $G^{+}$into lat. $\left(P \mathscr{U}^{(1)}\right)$, the lattice of normal subgroups of the group $\mathscr{U}^{(1)} / \mathscr{U}^{(1)} \cap$ $Z\left(\mathscr{U}^{(1)}=[\mathscr{U}, \mathscr{U}]\right)-$ One can also observe that $\mathscr{U}_{g}$ is of the form $U_{g}(V)$ for an appropriate space $V$. 
I proceed to show that if $g>0$, then the subgroup [[ $\left.\left.\mathscr{U}_{g}, \mathscr{U}_{g}\right], \mathscr{U}_{g}\right]$ contains some unitary $\gamma$ such that $w(\gamma-1)=3 g$. To begin with, I assert that $\mathscr{U}_{g}$ contains some unitary $\sigma$ such that $w(\sigma-1)=g$. Since $g>0=$ $w(2)$ it suffices to find some skew-symmetric $\phi$ in $D$ such that $w(\phi)=g$. For then if $\sigma=(1-\phi / 2) /(1+\phi / 2)$ then $w(\sigma-1)=w(\phi)=g$. Deny the assertion about $\phi$. This means that $w(\phi) \neq g$ for every skew-symmetric $\phi$ in $D$. Pick $a \in D$ such that $w(a)=g$. Since $w\left(a-a^{*}\right)=w(a)+$ $w\left(1-a^{-1} a^{*}\right)$ and since $a-a^{*}$ is skew-symmetric it follows that $w\left(1-a^{-1} a^{*}\right) \neq 0$. Since $1-a^{-1} a^{*} \in R$, it follows that $a^{-1} a^{*} \equiv 1$ $(\bmod J)$, for every $a \in D$ such that $w(a)=g$. If $t$ is any unit in $R$ then $w(a t)=w(a)=g$. Hence, $(a t)^{-1}(a t)^{*} \equiv 1(\bmod J)$. Then

$$
t^{-1} a^{-1} t^{*} a^{*}=(a t)^{-1}(a t)^{*} \equiv 1 \text {. }
$$

Left multiplying the preceding congruence by $t$ this gives

$$
a^{-1} t^{*} a^{*} \equiv t \quad(\bmod J)
$$

Thus

$$
a^{-1} t^{*} a=a^{-1} t^{*} a^{*}\left(a^{*-1} a\right)=a^{-1} t^{*} a^{*}\left(a^{-1} a^{*}\right)^{-1} \equiv t 1=t .
$$

Changing $t$ to $t^{*}$ this gives $a^{-1} t a \equiv t^{*}$ or $t^{a} \equiv t^{*}$. If $a$ is replaced by $a t_{1}$, where $t_{1}$ is another unit in $R$ then

$$
t^{*} \equiv t^{a}=t^{a t_{1}}=\left(t^{a}\right)^{t_{1}} \equiv t^{* t_{1}} \text {. }
$$

As this holds for every pair of units $t^{\prime}$ and $t_{1}$ it would follow that $R / J=\bar{D}$ is commutative, which is nonsense. This shows that there is $\phi=-\phi^{*}$ such that $w(\phi)=g$. Apply Theorem 4.3.1 to the pair $\sigma_{1}=\phi_{1}$, $\tau_{1}=\phi_{2}$. There are $u$ and $v$ symmetric units such that if $\phi_{1}=u \phi u$ and $\phi_{2}=v \phi v$ then $w\left(\left(\phi_{1}, \phi_{2}\right)\right)=w\left(\phi_{1}\right)+w\left(\phi_{2}\right)$. By construction $\phi_{1}$ and $\phi_{2}$ are skew-symmetrics and $w\left(\phi_{1}\right)=w\left(\phi_{2}\right)=w(\phi)=g$. Apply now Theorem 4.3.2 to the pair $\sigma_{2}=\phi_{1}, \tau_{2}=\phi_{2}$ to get a third skew-symmetric $\phi_{3}$ such that $w\left(\left(\phi_{1}, \phi_{2}\right), \phi_{3}\right)=3 g$, where $\phi_{3}=u^{\prime} \phi_{1} u$ or $v^{\prime} \phi_{2} v^{\prime}$ for some symmetric units $u^{\prime}$ and $v^{\prime}$ in $R$. Again, $w\left(\phi_{3}\right)=w\left(\phi_{1}\right)$ or $w\left(\phi_{2}\right)$. Since $w\left(\phi_{1}\right)=w\left(\phi_{2}\right)=g$ it follows that $w\left(\phi_{3}\right)=g$. If

$$
\sigma_{i}=\left(1-\phi_{i} / 2\right) /\left(1+\phi_{i} / 2\right)
$$

then by Theorem 4.3.3, the $\sigma_{i}$ are all unitaries such that $w\left(\sigma_{1}\right)=w\left(\sigma_{2}\right)=$ $w\left(\sigma_{3}\right)=g$ and $w\left(\left[\left[\sigma_{1}, \sigma_{2}\right], \sigma_{3}-1\right]\right)=3 g$, as desired.

It is now easy to establish the one-to-one requirement about the mapping $g \rightarrow P \mathscr{U}_{g}^{(1)}$. For if $P \mathscr{U}_{g_{1}}^{(1)}=P \mathscr{U}_{g_{2}}^{(1)}$ then $\left[\left[\mathscr{U}_{g_{1}}, \mathscr{U}_{g_{1}}\right], \mathscr{U}_{g_{1}}\right]=$ $\left[\left[\mathscr{U}_{g_{2}}, \mathscr{U}_{g_{2}}\right], \mathscr{U}_{g_{1}}\right]$. If the $\sigma_{i}$ are chosen as in the preceding then since $\gamma=\left[\left[\sigma_{1}, \sigma_{2}\right], \sigma_{3}\right] \in\left[\left[\mathscr{U}_{g_{1}}, \mathscr{U}_{g_{1}}\right], \mathscr{U}_{g_{1}}\right]$ it follows that $\gamma \in\left[\left[\mathscr{U}_{g_{2}}, \mathscr{U}_{g_{2}}\right], \mathscr{U}_{g_{1}}\right] \subset$ $\mathscr{U}_{2 g_{2}+g_{1}}$. Thus $w(\gamma-1) \geq 2 g_{2}+g_{1}$, and, consequently, $3 g_{1}=w(\gamma-1)$ $\geq 2 g_{2}+g_{1}$ giving $2 g_{1} \geq 2 g_{2}$ or $g_{1} \geq g_{2}$. By symmetry $g_{2} \geq g_{1}$ and, hence, $g_{1}=g_{2}$, which completes the proof. 
Theorem 4.3.4 can be put to work for the considered 1-dimensional spaces $V$. At no extra cost in the arguments the results apply to any 0 -elliptic space $V$. This is the

Theorem 4.3.5. Assume that ( $D ; * ; w$ ) is such that (i) $1 / 2 \in D$ and (ii) $\operatorname{dim}_{\bar{Z}} \bar{D}>4$. If $V$ is any 0-elliptic non-degenerate space over $D$ then $P \pi_{1}=g \in(0, \rightarrow) \mapsto\left[U_{t}, u_{g}\right] /[U, U] \cap Z$ is a dual isomorphism from $G^{+}$ into lat. $\left(P U^{(1)}\right)$, the lattices of normal subgroups of the projective derived unitary group of $V$.

Proof. By Fact 4.1.12, it suffices to establish the theorem in the case $\operatorname{dim}_{D} V=1$. As a second reduction, to show the theorem in the latter case it is the same as showing Theorem 4.3 .4 for $*$ replaced by the co-gredient involution $(s)$, where $s$ is any non-zero element in $D$ which is represented by the form. Indeed, for such an $s$ it is clear that $V={ }_{D} D$ (up to isometry), relative to the form $x \cdot y=x s y^{*}$. Here $L=L_{D}(D)=D_{\mathscr{R}}, x_{\mathscr{R}}^{*}=x_{\mathscr{R}}^{(s)}$ and $x_{\mathscr{R}} w \geq g \Leftrightarrow w(x) \geq g$. From this $\pi_{0}(g)=\left(\mathscr{U}_{g}(D ;(s))\right)$. Since $x \rightarrow x_{\mathscr{R}}$ is an isomorphism from $(D ;(s))$ onto $(L ; *)$ with correspondence of the subgroups $\pi_{0}(g)$ and $\mathscr{U}_{g}(D ;(s))$ it follows that the relations $\left[\pi_{1}(g), \pi_{0}\left(g_{1}\right)\right]=\left[\pi_{1}\left(g_{2}\right), \pi_{0}\left(g_{1}\right)\right]$, and $\left[\pi_{1}\left(g_{2}\right), \pi_{0}\left(g_{2}\right)\right]=\left[\pi_{1}\left(g_{1}\right), \pi_{0}\left(g_{2}\right)\right]$ are equivalent to the corresponding relations where $\pi_{0}(g), \pi_{1}(g)$ are replaced respectively by $\mathscr{U}_{g}(D ;(s)),\left[\mathscr{U}_{g}(D ;(s)), \mathscr{U}_{g}(D ;(s))\right]$. Since the former relations follow from the relation $P \pi_{1}\left(g_{1}\right)=P \pi_{1}\left(g_{2}\right)$. (Fact 4.1.11) and since $g_{1}=g_{2}$ follows from the former relations (see the proof of Theorem 4.3.4) it is now evident that $P \pi_{1}$ is one-to-one provided $(D ;(s) ; w)$ has the same qualification as $(D ; * ; w)$. Indeed, $w$ remains an $(s)$-valuation and the residue division ring has not changed.

Question. (Open.) Is $g \rightarrow P U_{g}^{(r)}$ again one-to-one where $g>0$ and $(D ; * ; w)$ is such that $1 / 2 \in \bar{D}$ and $\operatorname{dim}_{\bar{Z}} \bar{D}>4$ ? If yes, what if $\bar{D} \neq \bar{Z}$ ?

The assumption $\bar{D} \neq \bar{Z}$ cannot be dropped if one is to establish that $g \rightarrow U_{g}$ is one-to-one-let alone $g \rightarrow P u_{g}^{(r)}$. This is underscored in the

REMARK 4.3.8. Let $D$ be any finite-dimensional-valued division ring such that $\bar{D}$ is the real field and $G$ is the ordered additive group of integers. Assume, further, that $D$ carries an involution of the first kind. Then $(D ; * ; w)$ is a $*$-valued division ring for which $g \rightarrow \mathscr{U}_{h}(D ; *)$ is not one-to-one on each open segment $(n, \rightarrow)$.

Proof. The involution $*$ automatically verifies the assumption $w\left(x^{*}\right)$ $=w(x)$ as this was observed earlier in $\S 1$ (Dieudonné). Since $\bar{D}$ is the real 
field it follows that the induced involution of $\bar{D}$ is the identity. Equivalently $0 \notin w(\mathscr{K}(D ; *))$, where $w(\mathscr{K}(D ;(*)))=\left\{w(x) \mid x=-x^{*} \in D\right\}$. Since $w(\mathscr{K}(D ; *))+2 z \subset w(\mathscr{K}(D ; *))$ it follows that $w(\mathscr{K}(D ; *))$ consists only of odd integers. If $r_{0}$ is the least positive integer then $w(\mathscr{K}(D ; *))=r_{0}+2 Z$. If $(n, \rightarrow)$ is any open segment then for some $m$ large enough $r_{1}=r_{0}+2 m \in w(\mathscr{K}(D ; *))$ follows. By construction, if $\mathscr{K}_{r_{1}}=\mathscr{K}_{r_{1}}(D ; *)$ and $\mathscr{K}_{r_{2}}(D ; *)$ then $\mathscr{K}_{r_{1}}=\mathscr{K}_{r_{2}}$, where $x_{2}=r_{1}+1$. Hence, $\mathscr{U}_{r_{2}}=\mathscr{U}_{r_{1}}$, where $r_{1} \neq r_{2}$ and $r_{1}, r_{2} \in(n, \rightarrow)$. Thus $g \rightarrow \mathscr{U}_{g}$ is not one-toone, where $g \in(n, \rightarrow)$.

4.4. The general mapping $g \in P U_{g}^{(*)}$. Denote by $\pi_{r}$ the mapping sending $g \in(w(2), \rightarrow)$ onto $U_{g}^{(r)}$ and by $P \pi_{r}$ the one sending $g$ onto $P U_{g}^{(r)}=\pi_{r}(g) / U^{(r)} \cap Z$. Only a scratch of results will be offered here. Yet, I will have to rely on my work [5], which deals with the normal subgroup structure of $P U^{(r)}$, where $V$ is any non-isotropic unitary space over a division ring $D$ containing at least elements. I will begin with a list of exceptional cases labelled cases (A) through (C):

Case (A). $V$ is a unitary space of dimension $1, \operatorname{dim}_{Z} D \leq 4$ and $(D ; *)$ is commutative.

Case (B). $V$ is 2-dimensional orthogonal space.

Case (C). $V$ is 1-dimensional and $\operatorname{dim}_{Z} D=16$. There follow results established in [4].

Fact 4.4.1 ([4]). Let $V$ be any non-isotropic unitary space over a division ring $D$ containing at least 7 elements and suppose that $V$ is not as in cases (A) or (B). If $N$ is any normal subgroup of the unitary group $U$ of $V$ then:

(1) If $N$ is abelian then, in fact, $N \subset Z$.

(2) If $N$ is not abelian then the centralizer of $N$ in $L$ is precisely $Z$.

Fact 4.4.2 ([4]). Let $V$ be as in Fact 4.4.1. If $N$ is any normal subgroup of $U$ such that $[N, N] \subset Z$ then, in fact, $N \subset Z$.

Fact 4.4.3 ([4]). Let $V$ be as in Fact 4.4 .1 and suppose, further, that $V$ is not as in Case (C). If $N$ and $N^{\prime}$ are normal subgroups of $U$ such that $N \cap N^{\prime} \subset Z$ then $N \subset Z$ or $N^{\prime} \subset Z$. 
Fact 4.4.4 ([4]). Let $V$ be as in Fact 4.4.3. If $N$ is any normal subgroup of $U$ not contained in $Z$ and if $N^{\prime}$ is a normal subgroup of a normal subgroup of $U$ such that $\left[N^{\prime}, N\right] \subset Z$ then $N^{\prime} \subset Z$.

If $V$ is any non-degenerate elliptic space it is clear that $V$ is non-isotropic and that the ground division ring $D$ contains at least 7 elements. In fact, since the valuation $w$ is not trivial it follows that $D$ cannot be algebraic over a finite field. Thus Facts 4.4.1 through 4.4.4 will apply. To apply them to the congruence subgroups $U_{h}$ one is hinted (see Fact 4.4.1) to find the centralizer of $U_{g}$ in $L$. From Fact 4.4.1, $C\left(U_{g}\right)=Z$ unless $U_{g} \subset Z$. By an adaptation of $\S 2$, Theorem 2.17 , one can show the first part of the

THEOREM 4.4.5. Let $V$ be any nondegenerate elliptic space, which is not as in Cases (A) or (B). Then:

(1) The mapping $P \pi_{0}=g \in(w(2), \rightarrow) \mapsto$ lat. $\left(U_{g} / U \cap Z\right)$ assumes non-trivial values.

(2) The mapping $P \pi_{r}=g \mapsto U_{g}^{(r)} / U^{(r)} \cap Z$ is as in $1(r \geq 0)$

(3) The range of $P \pi_{r}$ is an infinite chain of lat. $\left(P U^{(r)}\right)$.

Proof. (1) To say that $P U_{g} \neq 1$ is to say that $U_{g} \not \subset Z$, which holds for every $g \in G$.

(2) To say that $P U_{g}^{(r)}=1$ is to say that $U_{g}^{(r)} \subset Z$. By Fact 4.4 .2 follows $U_{g}^{(r-1)} \subset Z$. Step by step $U_{g} \subset Z$ follows, which is ruled out.

(3) By construction Range $\left(P \pi_{r}\right)$ is a chain in lat. $\left(P U^{(r)}\right)$. I claim that the infimum of Range $\left(P \pi_{r}\right)$ is the identity subgroup of $P U^{(r)}$. For let $P N$ be this infimum. Then

$$
N=\bigcap_{g>w(2)} U_{g}^{(r)} \cdot\left(Z \cap U^{(r)}\right)
$$

Now,

$$
\begin{aligned}
{[N, N] } & \subset \bigcap_{g>w(2)}\left[U_{g}^{(r)}\left(Z \cap U^{(r)}\right), U_{g}^{(r)}\left(Z \cap U^{(r)}\right)\right] \\
& =\bigcap_{g>w(2)}\left[U_{g}^{(r)}, U_{g}^{(r)}\right]=\bigcap_{g>w(2)} U_{g}^{(r+1)} \subset \bigcap_{g>w(2)} U_{t} \\
& =\bigcap_{g \in G} U_{g}=1 .
\end{aligned}
$$

Thus $N$ is abelian. Since $N$ is a normal subgroup it follows from Fact 4.4.1 that $N \subset Z$ so that $P N=N / U^{(r)} \cap Z=1$. If now $\operatorname{Range}\left(P \pi_{r}\right)$ were finite then since $\operatorname{Range}\left(P \pi_{r}\right)$ is a chain it would follow that this set has a first member, say, $P U_{g_{0}}^{(r)}=P N=1$, contradicting 2 . 
THEOREM 4.4.6. Let $V$ be any non-degenerate elliptic space other than in cases (A), (B) or (C). If $1 \neq P N$ is any member of lat. $\left(P U^{(r)}\right)$ and if $P X$ is any member of Range $\left(\pi_{r}\right)$ then $P N \cap P X \neq 1$.

Proof. Deny the conclusion of the theorem. For some normal subgroup $N$ of $U^{(r)}, N \not \subset Z$, and some $g>w(2), P N \cap P U_{g}^{(r)}=1$. Equivalently, $\left[N, U_{g}^{(r)}\right] \subset Z$. Now $C\left(U_{g}^{(r)}\right)=Z$ and $N \triangleleft U^{(r)} \triangleleft U$. From Fact 4.4.4 follows $N \subset Z$, a contradiction.

As an application of the foregoing theorems:

THEOREM 4.4.7. Let $V$ be any non-degenerate elliptic space other than in cases (A), (B) or (C) and assume that one at least of the following requirements hold true.

(1) $*$ is an involution of the first kind.

(2) The restriction of $w$ on $Z$ is the trivial valuation.

(3) $D$ is finite-dimensional over its center.

Then for every natural number $r \neq 0$ the projective group $P U^{(r)}$ contains no torsion normal subgroups $\neq 1$.

Proof. It suffices to find some torsion-free member of $\operatorname{Range}\left(\pi_{r}\right)$ (Theorem 4.4.6). Define $g_{0}$ to be the element 0 in $G$ if $\operatorname{charac}(D) \neq 0$; if, to the contrary, charac $(D)=0$, let $g_{0}=w(g)$, where $p=\operatorname{charac}(\bar{D})$. If $g_{1}>\max \left(g_{0}, w(2)\right)$ then by $\$ 2$, Theorem $2.19, U_{g_{1}}$ is torsion-free. It is to be shown that $P U_{g_{1}}^{(r)}$ is torsion-free, where $r \neq 0$. For let $\phi \in U_{g_{1}}^{(r)}$ be such that $\phi^{m} \in Z$.

Case 1. Here $U \cap Z=\{1,-1\}$ so that $\phi$ is a root of unity. Thus $\phi=1$. Thus $P U_{g_{1}}^{(r)}$ is torsion-free.

Case 2. If $\phi^{m}=z \in Z$ then since $\phi^{m} \in U_{g_{1}}$ it follows that $(z-1) w \geq$ $g_{1}>0$. Equivalently, $w(z-1)>0(\S 2$, Theorem 2.4.2.). Thus $z-1=0$ or $z=1$. Thus $\phi=1$ and, hence, $P U_{g_{1}}^{(r)}$ is torsion-free.

Case 3. By standard argument $U^{(1)} \cap Z$ consists only of roots of unity. From $\phi^{m} \in U_{g_{1}}^{(r)} \subset U^{(1)}$ follows that $\phi$ is a root of unity. From this $\phi=1$ so that $P u_{1}^{(r)}$ is torsion-free.

THEOREM 4.4.8. Let $V$ be any non-degenerate elliptic space other than in cases (A) or (B). Every torsion normal subgroup of $U$ is central. 
Proof. For let $N$ be any torsion normal subgroup of $U$. If $g_{1}$ is defined as in the proof of Theorem 4.4.7, then $U_{g_{1}}$ is torison-free. Thus $N \cap U_{g_{1}}=1$ and, hence, $N \subset C\left(U_{g_{1}}\right)=\mathrm{Z}$.

Question. (Open.) Does Theorem 4.4 .7 carry over for any ground division ring $D$ ? What about the case $r=0$ ?

To close let me add one more question.

Question (Open.) Is the range of $\pi_{r}$ large enough so as for every $\mathrm{Z} \not \subset N<U^{(r)}, P N \supset P U_{g}^{(r)}$, some $g>w(2)(r \neq 0)$ ?

The preceding question can be answered in the negative in case $r=0$. This uses a counterexample in [3].

Acknowledgments. I wish to express my thanks to the referee of a first version of this work whose many suggestions inspired in part the present verison, to $H$. Rossi, editor, who allowed extra time for the revision, to Carleton University for granting me the sabbatical year '83-84 and to the Natural Sciences and Engineering Research Council of Canada (Grant A7876) for partial support.

\section{REFERENCES}

[1] E. Artin, Geometric Algebra, Interscience Publishers, Inc., New York, 1954.

[2] M. Chacron, Unitaries in simple artinian rings, Canad. J. Math., Vol. XXI, No. 3, (1979), 512-517.

[3] _ On a conjecture of I. N. Herstein, J. Algebra (to appear).

[4] C C-Orderable division Rings II,

[5] Normal subgroup strucgture of the unitary group for non-isotropic unitary space, (manuscript).

[6] J. Dieudonné, On the structure of unitary groups, Proc. Amer. Math. Soc., (1952), 367-385.

[7] H. Gross, Quadratic Forms on Infinite Dimensional Vector Spaces, Progression Mathematics, Birkhauser, 1979.

[8] I. N. Herstein, Rings with Involution, University of Chicago Press (1976), Chicago

[9] _ A unitary version of the Brauer-Cartan-Hua theorem, J. Algebra, to.32, (1974), 555-560.

[10] S. S. Holland (Jr.), Orderings and square roots in *-fields, J. Algebra, No. 46, (1977), 207-219.

[11] , *-valuations and ordered *-fields, Trans. Amer. Math. Soc., 267, No. 1, (1980), 219-243.

[12] N. Jacobson, Basic Algebra II, W. H. Freeman and Company, San Francisco 1980.

[13] I. Kaplansky, Linear Algebra and Geometry, Allyn and Bacon Inc. 
[14] E. M. Patterson, On the radicals of rings of row-finite matrices, Proc. Roy. Sic. Edin. A, 66 (1962), 42-46.

[15] O. G. S. Schilling, The Theory of Valuations, Mathematical Surveys, No. 4 (1950).

[16] W. R. Scott, Group Theory, Prentice-Hall, Inc. Englewood Cliffs, New Jersey, 1964.

Received November 2, 1981 and in revised form May 3, 1984.

\section{CARLETON UNIVERSITY}

Ottawa, Ontario, Canada 



\section{PACIFIC JOURNAL OF MATHEMATICS EDITORS}

\author{
V. S. VARADARAJAN (Managing Editor) \\ University of California \\ Los Angeles, CA 90024 \\ Charles R. DePrima \\ California Institute of Technology \\ Pasadena, CA 91125 \\ R. FINN \\ Stanford University \\ Stanford, CA 94305
}

\author{
HeRmanN FlaschKa \\ University of Arizona \\ Tucson, AZ 85721 \\ Ramesh A. Gangolli \\ University of Washington \\ Seattle, WA 98195 \\ ROBION KIRBY \\ University of California \\ Berkeley, CA 94720
}

C. C. MOORE

University of California

Berkeley, CA 94720

H. SAMELSON

Stanford University

Stanford, CA 94305

HAROLD STARK

University of California, San Diego

La Jolla, CA 92093

ASSOCIATE EDITORS
R. ARENS
E. F. BECKENBACH
B. H. NeUmanN
F. WOLF
K. YosHIDA (1906-1982)

\section{SUPPORTING INSTITUTIONS}

UNIVERSITY OF ARIZONA

UNIVERSITY OF BRITISH COLUMBIA

CALIFORNIA INSTITUTE OF TECHNOLOGY

UNIVERSITY OF CALIFORNIA

MONTANA STATE UNIVERSITY

UNIVERSITY OF NEVADA, RENO

NEW MEXICO STATE UNIVERSITY

OREGON STATE UNIVERSITY
UNIVERSITY OF OREGON

UNIVERSITY OF SOUTHERN CALIFORNIA

STANFORD UNIVERSITY

UNIVERSITY OF HAWAII

UNIVERSITY OF TOKYO

UNIVERSITY OF UTAH

WASHINGTON STATE UNIVERSITY

UNIVERSITY OF WASHINGTON 


\section{Pacific Journal of Mathematics}

Vol. 119, No. $1 \quad$ May, 1985

Maurice Chacron, Nonisotropic unitary spaces and modules with

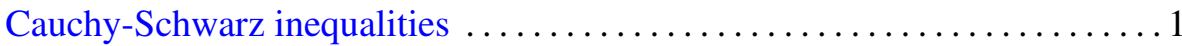

Myriam Dechamps-Gondim, Françoise Piquard and H. Queffélec, On

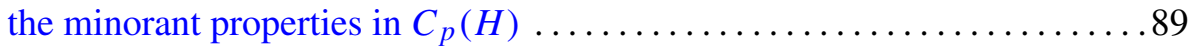

Klaus Floret and V. B. Moscatelli, On bases in strict inductive and projective limits of locally convex spaces .................... 103

Norman Joseph Goldstein, Degenerate secant varieties and a problem on

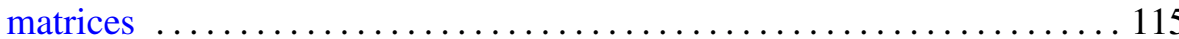

Harold Morris Hastings and Stefan Waner, $G$-bordism with singularities

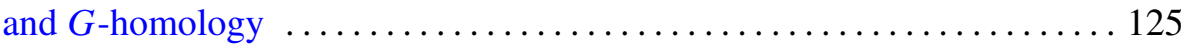

Takesi Isiwata, Clopen realcompactification of a mapping ........... 153

Hisao Kato, Concerning hyperspaces of certain Peano continua and strong

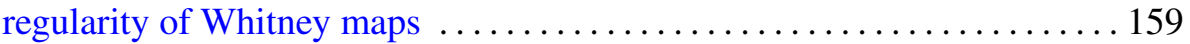

Elyahu Katz and Sidney Allen Morris, Free products of topological groups

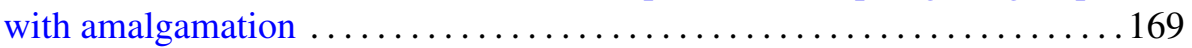

Kyewon Koh Park, Nice dense subsets for ergodic flows and Bernoulli

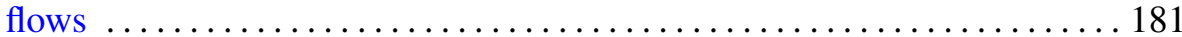

Francis Pastijn and Peter George Trotter, Lattices of completely regular

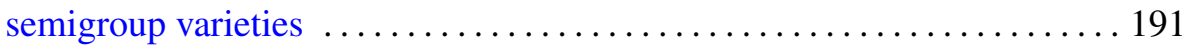

Rae Michael Andrew Shortt, Reticulated sets and the isomorphism of analytic powers

David A. Stegenga and Kenneth R. Stephenson, Generic covering properties for spaces of analytic functions

M. V. Subba Rao and R. Sitaramachandra Rao, On some infinite series of

L. J. Mordell and their analogues 